\title{
The regularity of Special Legendrian Integral Cycles
}

\author{
Costante Bellettini AND TRISTAN Rivière
}

\begin{abstract}
Special Legendrian Integral Cycles in $S^{5}$ are the links of the tangent cones to Special Lagrangian integer multiplicity rectifiable currents in CalabiYau 3-folds. We show that Special Legendrian Cycles are smooth except possibly at isolated points.
\end{abstract}

Mathematics Subject Classification (2010): 49Q15 (primary); 32Q25 (secondary).

\section{Introduction}

Some years ago, in a survey paper [5], S.K. Donaldson and R.P. Thomas gave a fresh boost to the analysis of non-linear gauge theories in geometry by exhibiting heuristically links between some invariants in complex geometry and spaces of solutions to Yang-Mills equations in dimensions higher than the usual conformal 4 dimensions for these equations. In [22] G. Tian described the loss of compactness of sequences of some Yang-Mills Fields in dimension larger than 4. This loss of compactness arises along $(n-4)$-rectifiable objects, called the blow-up sets. It plays a crucial role in the compactification procedure of the space of the solutions of $\Omega$-anti-self-dual instantons (the generalisation of the usual 4-dimensional instantons to dimensions larger than 4).

Can one expect the blow-up set to be more than just rectifiable? What is its exact nature?

At such a level of generality this question is wide open and difficult. The situation is better understood for some sub-classes of solutions: one example is given by the so-called $S U$ (4)-Instantons in a Calabi-Yau 4-fold. The concentration set is, in this case, the carrier of a calibrated rectifiable cycle. Among these cycles we find for instance the Special Lagrangian Integral Currents. This provides one possible field of application for Special Lagrangian Geometry or calibrated geometries in general.

The first author was partially supported by the Swiss Polytechnic Federal Institute Graduate Research Fellowship ETH-01 09-3 and by the Swiss National Science Foundation Graduate Research Fellowship 200021-126489.

Received July 2, 2009; accepted in revised form October 21, 2010. 
Further reasons for studying Special Lagrangians come from String Theory, more precisely from Mirror Symmetry. According to this model, our universe is a product of the standard Minkowsky space $\mathbb{R}^{4}$ with a Calabi-Yau 3-fold $Y$. Based on physical grounds, the so called SYZ-conjecture (named after Strominger, Yau and Zaslov) expects, roughly speaking, that this Calabi-Yau 3-fold can be fibrated by (possibly singular) Special Lagrangians, whence the interest in understanding the singularities of a Special Lagrangian current. The compactification of the dual fibration should lead to the mirror partner of $Y$. See the survey paper by Joyce [12] for a more thorough explanation.

We remark also that, as all calibrated geometries (see [10] or [11]), Special Lagrangian Geometry provides examples of volume-minimizing submanifolds or currents; Special Lagrangians are a particularly large family. Having such examples helps the understanding of the possible singular behaviour of such minimizers.

General description of the problem: setting and results. In the complex Euclidean space $\mathbb{C}^{3}$ with the standard coordinates $z=\left(z_{1}, z_{2}, z_{3}\right), z_{i}=x_{i}+i y_{i}$, consider the constant differential 3-form

$$
\Omega=\operatorname{Re}\left(d z^{1} \wedge d z^{2} \wedge d z^{3}\right)
$$

This is the so called Special Lagrangian calibration, introduced and analysed in [10]. We recall some notions from calibrated geometry, referring to the quoted paper for a broader exposition. Given a $m$-form $\phi$ on a Riemannian manifold $(M, g)$, the comass of $\phi$ is defined to be

$$
\|\phi\|^{*}:=\sup \left\{\left\langle\phi_{x}, \xi_{x}\right\rangle: x \in M, \xi_{x} \text { is a unit simple } m \text {-vector at } x\right\} \text {. }
$$

A form $\phi$ of comass one is called a calibration if it is closed $(d \phi=0)$; when it is non-closed it is referred to as a semi-calibration.

Let $\phi$ be a calibration or a semi-calibration; among the oriented $m$-dimensional planes that constitute the Grassmannians $G\left(m, T_{x} M\right)$, we pick those that (represented as unit simple $m$-vectors) realize $\left\langle\phi_{x}, \xi_{x}\right\rangle=1$ and define the set $\mathcal{G}(\phi)$ of $m$-planes calibrated by $\phi$ :

$$
\mathcal{G}(\phi):=\cup_{x \in M}\left\{\xi_{x} \in G\left(m, T_{x} M\right):\left\langle\phi_{x}, \xi_{x}\right\rangle=1\right\} .
$$

In other words, these are exactly the $m$-planes on which $\phi$ agrees with the $m$-volume form.

We recall now the notion of calibrated cycle. For definitions and notations from Geometric Measure Theory we refer to [7] or [8].

An integral $m$-cycle $C$ in $M$ is an integer multiplicity rectifiable current of di-

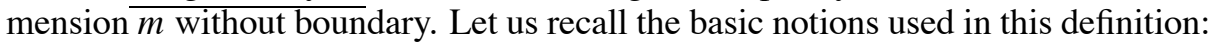

(i) Rectifiability: there is a countable family of oriented $C^{1}$ submanifolds $N_{i}$ of dimension $m$ in $M$; in each of them we take a $\mathcal{H}^{m}$-measurable subset $\mathcal{N}_{i}$, so that the $\mathcal{N}_{i}$-s are disjoint; the union $\mathcal{C}=\cup_{i} \mathcal{N}_{i}$ is a so-called oriented rectifiable set. 
$\mathcal{C}$ possesses an oriented approximate tangent plane $\mathcal{H}^{m}$-a.e. (see [7] or [8]). On $\mathcal{C}$ an integer valued and locally summable multiplicity function $\theta$ is given, $\theta \in L_{\text {loc }}^{1}(\mathcal{C} ; \mathbb{Z})$; the action of the current $C$ on any $m$-form $\psi$, that is smooth and compactly supported in $M$, is given by

$$
C(\psi)=\int_{\mathcal{C}} \theta(x)\left\langle\psi_{x}, \xi_{x}\right\rangle d \mathcal{H}^{m}(x),
$$

where $\xi_{x}$ is the oriented tangent at $x$ represented as a unit simple vector.

(ii) Closedness: the boundary $\partial C$ of the current is $0^{1}$. Explicitly: for any smooth $(m-1)$-form $\alpha$, that is compactly supported in $M$,

$$
(\partial C)(\alpha):=C(d \alpha)=0 .
$$

The class of integer-multiplicity, rectifiable currents of dimension $m$ in $M$ is denoted by $\mathcal{R}_{m}(M)$. The support $\operatorname{spt}(C)$ of the current is defined as the complement of the open set

$\cup\{A: A$ is open and $C(\psi)=0$ for all $m$-forms $\psi$ compactly supported in $A\}$.

Without loss of generality one can assume $\theta$ to be strictly positive: for that purpose it is enough to choose the appropriate orientation for the oriented rectifiable set and neglect the part where $\theta=0$. With this in mind, one can always express the action of a rectifiable current $C$ by means of a rectifiable set $\mathcal{C}$ on which a multiplicity function $\theta \in L_{\text {loc }}^{1}(\mathcal{C} ; \mathbb{N} \backslash\{0\})$ is given: this underlying rectifiable set $\mathcal{C}$ is referred to as the carrier of the current $C$.

We recall the notions of Smooth Points and Singular Points. A point $x \in \mathcal{C}$ is said to be a smooth point if there is a ball $B_{r}(x)$ in which the current acts as a smooth $m$-submanifold $\mathcal{V}$, i.e. if there is some constant $N \in \mathbb{N}$ such that for any smooth $m$-form $\psi$ compactly supported in $B_{r}(x)$

$$
C(\psi)=N \int_{\mathcal{V}} \psi
$$

The set of smooth points is open in $\mathcal{C}$ by definition; its complement in $\mathcal{C}$ is called the singular set of $C$, denoted by Sing $C$.

For a current in $\mathcal{R}_{m}(M)$, at $\mathcal{H}^{m}$-almost every point $x \in \mathcal{C}$ denote by $T_{x} \mathcal{C}$ the $m$-dimensional oriented approximate tangent plane to the underlying rectifiable set $\mathcal{C}$; given a (semi)-calibration $\phi, C$ is said to be calibrated by $\phi$ if

$$
\text { for } \mathcal{H}^{m} \text {-almost every } x, \operatorname{sign}(\theta) T_{x} \mathcal{C} \in \mathcal{G}(\phi) .
$$

When $\phi$ is a closed form, then a current calibrated by $\phi$ is locally homologically volume-minimizing; (closed) calibrations were introduced in the foundational paper [10].

1 The term cycle refers to the absence of boundary. 
Returning to our case, being $\Omega$ constant, it is obviously closed; as shown in [10] it has comass one. Currents in $\mathcal{R}_{3}\left(\mathbb{C}^{3}\right)$, calibrated by $\Omega$, are called Special Lagrangians. of $\Omega$ by

Let $N$ denote the radial vector field $N:=r \frac{\partial}{\partial r}$ in $\mathbb{C}^{3}$ and define the normal part

$$
\Omega_{N}:=\iota_{N} \Omega,
$$

where $\iota$ denotes the interior product. We will work in the sphere $S^{5} \subset \mathbb{C}^{3}$, with the induced metric. Consider the pull-back of $\Omega_{N}$ on the sphere via the canonical inclusion map $\mathcal{E}: S^{5} \hookrightarrow \mathbb{C}^{3}$ :

$$
\omega:=\mathcal{E}^{*} \Omega_{N}
$$

An easy computation shows that

$$
\omega=\operatorname{Re}\left(z_{1} d z^{2} \wedge d z^{3}+z_{2} d z^{3} \wedge d z^{1}+z_{3} d z^{1} \wedge d z^{2}\right) .
$$

$\omega$ is a 2 -form on $S^{5}$ of comass one. Indeed, $|N|=1$ on $S^{5}$ and for any simple 2-vector $\xi$ in $T S^{5}$

$$
|\omega(\xi)|=|\Omega(N \wedge \xi)| \leq\|N \wedge \xi\|=\|\xi\| .
$$

Equality is surely reached when $N \wedge \xi$ is a Special Lagrangian 3-plane, compare Proposition 1.3. We remark that both $\Omega$ and $\omega$ are $S U(3)$-invariant. As explained in [10, Section II.5] or [11, Section 2.2], $\omega$ is non-closed.

$\omega$ is referred to as the Special Legendrian semi-calibration. Rectifiable currents in $S^{5}$ calibrated by $\omega$ are called Special Legendrians.

Our main result is the following:

Theorem 1.1. An integer multiplicity rectifiable current $C$ without boundary calibrated by $\omega$ (this is called a Special Legendrian integral cycle) in $S^{5}$ can only have isolated singularities, therefore finitely many.

In other words: $C$ is, out of isolated points, the current of integration along a smooth Special Legendrian submanifold with smooth integer multiplicity.

Remark 1.2. This result is optimal. We will provide an example in the next section, see Remark 2.7.

Still from [10, Section II.5] or [11, Section 2.2], the 2-currents of $S^{5}$ on which $\omega$ restricts to the area form are exactly those such that the cone built on them is calibrated by $\Omega$ :

Proposition 1.3 ([10] or [11]). A rectifiable current $T$ in $S^{5}$ is a Special Legendrian if and only if the cone on $T$

$$
C(T)=\left\{t x \in \mathbb{R}^{6}: x \in T, t>0\right\}
$$

is Special Lagrangian. 
We know that Special Lagrangian currents (as a particular case of currents calibrated by a closed form) are (locally) homologically area-minimizing in $\mathbb{C}^{3}$; from [1] we know that volume-minimizing 3-cycles are smooth outside a set of Hausdorff dimension 1. In the case of a cone, this roughly translates into having radial lines of singularities, possibly accumulating onto each other. We establish here that there can only by a finite number of such lines.

We remark here that Special Lagrangians can be defined in general Calabi-Yau $n$-folds, see [12]; Special Lagrangians are known to possess tangent cones at all points (see [10, Section II.5]), and such cones are Special Lagrangian cones in $\mathbb{C}^{n}$. Thanks to Proposition 1.3, our result can be restated as follows:

Corollary 1.4. Tangent cones to a Special Lagrangian in a Calabi-Yau 3-fold have a singular set made of at most finitely many lines passing through the vertex.

From [20, Proposition 6.1.1], $T$ in $S^{5}$ is minimal, in the sense of vanishing mean curvature, if and only if $C(T) \subset \mathbb{C}^{3}$ is minimal. Therefore, Special Legendrians are minimal currents in $S^{5}$ (although not necessarily area-minimizing).

Relying on [1], Chang proved in [3] the corresponding regularity result for area-minimizing 2-dimensional currents.

One advantage coming from the existence of the calibration, as will be seen, is the fact that the current can locally be described as integration along a multivalued graph satisfying a first order elliptic PDE; the general problem of volumeminimizing currents, instead, requires an elliptic problem of order two, see [1] or [3]. It is also remarkable that the general regularity theory for mass-minimizing currents developed by Almgren is extremely hard; his Big Regularity Paper [1] comprises a thousand pages and it is therefore helpful to have shorter (and relatively easier) self-contained proofs of regularity results for some sub-classes of minimizing currents, such as Special Lagrangian currents or $J$-holomorphic currents (see $[17,18,21])$.

Proof. We will now give a sketch of our proof. The underlying structure is basically the same as in [21] and [17], where the regularity of $J$-holomorphic cycles in a 4dimensional ambient manifold was shown. In our case we have a fifth coordinate to deal with, which introduces new challenging difficulties, as will be seen.

A standard blow-up analysis tells us that at any point $x$ of $S^{5}$ the multiplicity function $\theta(x)=\lim _{r \rightarrow 0} \frac{M\left(C\left\llcorner B_{r}(x)\right)\right.}{\pi r^{2}}$ is ${ }^{2}$ an integer $Q$. The monotonicity formula (see [16] or [19]) tells us that, at any $x_{0}, \frac{M\left(C\left\llcorner B_{r}\left(x_{0}\right)\right)\right.}{r^{2}}$ is monotonically nonincreasing as $r \downarrow 0$, whence we get that $\theta$ is upper semi-continuous. Therefore the

2 For general integral cycles, the $\operatorname{limit}_{\lim } \rightarrow 0 \frac{M\left(C L B_{r}(x)\right)}{\pi r^{2}}$ exists a.e. and coincides with the absolute value $|\theta|$ of the multiplicity assigned in the definition of integer cycle. In our case $\lim _{r \rightarrow 0} \frac{M\left(C\left\llcorner B_{r}(x)\right)\right.}{\pi r^{2}}$ is well-defined everywhere, therefore we can choose (everywhere) this natural representative for $\theta$, after having chosen the correct orientation for the approximate tangent plane. 
set

$$
\mathcal{C} \leq Q:=\left\{x \in S^{5}: \theta(x) \leq Q\right\}
$$

is open in $S^{5}$. This allows a proof by induction of our result: indeed, the statement of Theorem 1.1 is local, so we can restrict the current to $\mathcal{C} \leq Q$ and consider increasing integers $Q$ (see the beginning of Section 5).

One key ingredient is the construction of families of 3-dimensional surfaces $\Sigma$ which locally foliate $S^{5}$ and that have the property of intersecting positively the Special Legendrian ones. As in [17], this algebraic property can be exploited to provide a self-contained proof of the uniqueness of tangent cones for our current. This result was proved for general semi-calibrated cycles in [16] and for general area-minimizing ones in [23] using a completely different approach ${ }^{3}$.

Further, the positiveness of intersection allows us to describe our current, locally around a point $x_{0}$ of multiplicity $Q$, as a $Q$-valued graph from a disk $D^{2} \subset \mathbb{C}$ into $\mathbb{R}^{3} \cong \mathbb{C} \times \mathbb{R}$. This means that we associate to each $z \in D^{2}$ a $Q$-tuple of points in $\mathbb{C} \times \mathbb{R}$. The $Q$-tuple is to be understood as unordered, i.e. as an element of the $Q$-th symmetric product of $\mathbb{C} \times \mathbb{R}$. It is not possible to find, globally on $D^{2}$, a coherent labeling of the multi-valued graph as a superposition of $Q$ functions.

The transition current $\rightarrow$ multi-valued graph is done by slicing the current with a "parallel family" of 3-surfaces $\Sigma$ of the type mentioned above: one must choose a good "direction" for the slicing, namely take a $\Sigma$ that is transverse to the tangent cone at $x_{0}$. This ensures, locally around $x_{0}$, the constancy of the intersection index when we move $\Sigma$ "parallel to itself". The intersection index, which counts intersections with signs, turns out to be constantly $Q$. But the sign of intersection is always positive, due to the property of the $\Sigma$ 's. This yields that the number of points at which the 3 -surfaces cross the current is exactly $Q$, taking multiplicities into account.

Currents of integration along multivalued graphs constitute one of the important objects of interest in Geometric Measure Theory. Multivalued graphs were introduced by Almgren in [1] for the study of Dirichlet-minimizing and volumeminimizing currents and were lately revisited in a new flavour in [4].

As we said above, the proof of Theorem 1.1 is done by induction on the multiplicity $Q$. Recall that by upper semi-continuity of the multiplicity, we already know that all points in a neighbourhood of a point of multiplicity $Q$ have multiplicity no higher than $Q$. Therefore, the inductive step is divided into two parts: in the first one we show that there is no possibility for an accumulation of singularities of multiplicity $Q$ to a singularity of the same multiplicity; in the second part we exclude accumulation of lower order singularities to a singularity of order $Q$.

First part of the inductive step. There is a situation in which, just by slicing techniques, it is possible to exclude the possibility that singular points of multiplicity $Q$ accumulate onto a point $x_{0}$ of the same multiplicity. This case occurs when the

3 The proof in [23] relies however on the area-minimality property which is not generally true for Special Legendrians. 
tangent cone at $x_{0}$ is not made of $Q$ times the same disk and will be referred to as easy case of non accumulation (see Theorem 4.6).

The case of a point with a tangent made of the same disk counted $Q$ times is considerably harder and leads to Theorem 5.1. Let us therefore focus on this case and see an overview of the several steps.

We introduce the first order PDEs (for the $Q$-valued graph) that describe the calibrating condition. These equations turn out to be, in appropriate coordinates, perturbations of the classical Cauchy-Riemann equations, but with three real functions and two real variables.

More precisely, we denote the $Q$-valued graph describing the current in a neighbourhood of a point of multiplicity $Q$ by

$$
\left\{\left(\varphi_{j}(z), \alpha_{j}(z)\right)\right\}_{j=1 \cdots Q}
$$

where $z=x+i y$ is the coordinate in the Disk $D^{2} \subset \mathbb{C}, \varphi_{i} \in \mathbb{C}$ and $\alpha_{i} \in \mathbb{R}$. Without loss of generality we can assume $\left(\varphi_{j}(0), \alpha_{j}(0)\right)=(0,0)$ for all $j=1, \cdots, Q$, so that we are centered at the origin of $D^{2} \times \mathbb{C} \times \mathbb{R}$.

The equations solved by the branches of $\left\{\left(\varphi_{j}(z), \alpha_{j}(z)\right)\right\}$ are as follows:

$$
\left\{\begin{array}{l}
\partial_{\bar{z}} \varphi_{j}=v\left(\left(\varphi_{j}, \alpha_{j}\right), z\right) \partial_{z} \varphi_{j}+\mu\left(\left(\varphi_{j}, \alpha_{j}\right), z\right) \\
\nabla \alpha_{j}=h\left(\left(\varphi_{j}, \alpha_{j}\right), z\right)
\end{array}\right.
$$

where $v$ and $\mu$ are smooth complex valued functions on $\mathbb{R}^{5}$ such that $v(0)=$ $\mu(0)=0$ and $h$ is a smooth $\mathbb{R}^{2}$-valued map on $\mathbb{R}^{5}$.

It is remarkable that if we were dealing with a single (Sobolev) solution $\varphi$ : $D^{2} \subset \mathbb{C} \rightarrow \mathbb{C} \times \mathbb{R}$ of the system above, then the regularity question would be easily answered by elliptic theory, yielding that $\varphi$ is $C^{\infty}$.

As soon as we have a multi-valued graph, even just 2-valued, singularities are actually allowed! Then we can restate Theorem 1.1 by saying that a singular behaviour for a multi-valued graph solving the system above is possible at most at isolated points.

We stress here that in order to get a $Q$-valued graph solving the system above we need to perform a careful choice of coordinates. Since this choice will require a lot of work, we digress shortly on its importance.

With general coordinates, induced by a slicing with arbitrary 3-dimensional surfaces, we would, in a first instance, lose the property of positive intersection and not any longer get a $Q$-valued graph. We could only associate to each $z \in D^{2} a$ set of points $\left\{A_{1}(z), \ldots, A_{P}(z), B_{1}(z), \ldots, B_{N}(z)\right\}$ with $P, N \in \mathbb{N}$ changing with $z$. The only thing that would be independent of $z$ would be the difference $P-N=Q$. The points $A_{i}$ would be those where there is a positive intersection with the slicing surfaces, the $B_{i}$-s those where this intersection is negative.

In addition to this, a further difficulty would arise. Writing equations for this "algebraic" $Q$-valued graph, we would find a supercritical equation, as explained in [18]. In comparison with the system (1.1), we would have a dependence on $\nabla \varphi_{j}$ 
inside $\mu$ and $v$. With such an equation, even for a single-valued graph, we could not perform bootstrapping in order to get regularity, and in our case of multiple values, the unique continuation argument (see below) would fail.

Let us go back to the proof. Using the PDEs (1.1) we prove a $\underline{W^{1,2} \text { estimate }}$ for the average $(\tilde{\varphi}, \tilde{\alpha})$ of the branches of our multivalued graph. We remark here that we give a proof of the $W^{1,2}$-estimate different than the one in [17], where the authors had the further hypothesis that $\operatorname{Sing} C$ was $\mathcal{H}^{2}$-negligible (see Theorem 5.9).

We make a key use of the so-called relative Lipschitz estimate (Theorem 4.7 and Corollary 5.8). This estimate tells us the following: taken a point $x_{0}$ of multiplicity $Q$ whose tangent cone is made of $Q$ times the same disk $D_{0}$, if there is a sequence of points $\left\{y_{n}\right\}$ of multiplicity $Q$ accumulating onto $x_{0}$ then the tangent cones at the points $y_{n}$ must flatten towards $D_{0}$ as $n \rightarrow \infty$ (see Figure 5.1).

The $W^{1,2}$-regularity of the average allows us to translate the issue of accumulation of singularities of multiplicity $Q$ into a problem of accumulation of zeros for a new $Q$-valued graph solving a PDEs system - equations (6.14) and (6.15), that is again a perturbation of the classical Cauchy-Riemann. The new multi-valued graph, described by (6.13), is obtained from the original one by subtracting the average, as illustrated in Figure 6.1. The $W^{1,2}$-regularity of the average is the minimum regularity required in order to get that the new $Q$-valued graph (6.13) still represents a boundaryless current in $D^{2} \times \mathbb{C} \times \mathbb{R}$ : this fact is crucial later for the essential integration by parts formulae (see Lemma 6.6).

Then by a suitable adaptation of the unique continuation argument used in

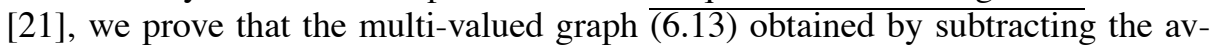
erage from each branch cannot have accumulating zeros, thereby concluding the first part of the inductive step. The proof is by contradiction. The argument requires a further modification of the multi-valued graph (see (6.16) and (6.18)): this trick allows to "focus attention" on an accumulating sequence of zeros. In order to get a $L^{\infty}$-bound for this multi-valued graph (6.18) we need the Lipschitz-type estimate of Corollary 5.8. Then we can use the partial integration allowed by Lemma 6.6 and get a contradiction thanks to the elliptic nature of the equations (6.19) and (6.20) satisfied by the multi-valued graph.

The techniques we employ to show the partial integration formulae for multivalued graphs are more typical of geometric measure theory; we also provide in Lemma 6.6 a step that was incomplete in [21].

Second part of the inductive step. Let $x_{0}$ be a point of multiplicity $Q$ such that, in a neighbourhood $B_{r}\left(x_{0}\right)$, the current $C$ is smooth except at points of multiplicity $\leq Q-1$ that are isolated in $B_{r}\left(x_{0}\right) \backslash\left\{x_{0}\right\}$ (this is what we have from the inductive assumption and from the first part of the inductive step). Then we aim to prove that it is not possible to have a sequence of such isolated singularities of multiplicity $\leq Q-1$ accumulating onto $x_{0}$ (this is the content of Theorem 7.1).

We use an homological argument inspired by the one used in [21], where the same statement was proved in the case of $J$-holomorphic cycles in a 4-manifold, although in our case the existence of the fifth coordinate induces new difficulties and a more involved argument. 
For the moment we just sketch the underlying idea, warning the reader that in Section 7 the formal proof will require new spaces and functions, different from those sketched here, and some delicate estimates.

As above, we denote the $Q$-valued graph describing the current in a neighbourhood of a singular point $x_{0}$ of multiplicity $Q$ by

$$
\left\{\left(\varphi_{j}(z), \alpha_{j}(z)\right)\right\}_{j=1 \cdots Q},
$$

and denote $\pi: D^{2} \times \mathbb{C} \times \mathbb{R} \rightarrow D^{2}$ the projection map. There is no loss of generality in taking $x_{0}=0$, the origin of $D^{2} \times \mathbb{C} \times \mathbb{R}$. We assume (inductive assumption + first part of the inductive step) that the multi-valued graph is smooth except at 0 and at a sequence of points (different from 0 ) having multiplicity $\leq Q-1$, isolated in $D^{2} \times \mathbb{C} \times \mathbb{R}$ and accumulating to $x_{0}$ (so we are arguing by contradiction to prove Theorem 7.1). Denote the projection onto $D^{2}$ of this sequence by $\left\{z_{j}\right\}$.

Roughly speaking, we would like to exhibit a continuous function $u: D^{2} \rightarrow$ $\mathbb{C}$, vanishing exactly on the set $\pi($ Sing $C)=\left\{0, z_{1}, \ldots z_{j}, \ldots\right\}$, such that when we observe $\frac{u}{|u|}$ on positively oriented loops in $D^{2} \backslash \pi$ (Sing $C$ ) the following hold:

(i) if the loop $\gamma$ encloses a point $z_{j}$ then the topological degree of $\frac{u}{|u|}: \gamma \rightarrow S^{1}$ on that loop is strictly positive;

(ii) for any loop $\gamma_{r}=\partial B_{r}(0)$ around the origin, the degree of $\frac{u}{|u|}: \gamma_{r} \rightarrow S^{1}$ is bounded from below by a constant $k \in \mathbb{Z}$ independent of $r$.

From these properties we could conclude Theorem 7.1 by the following homotopy argument.

Take any loop $\gamma_{r_{1}}=\partial B_{r_{1}}(0)$ lying in $D^{2} \backslash \pi(\operatorname{Sing} C)$ and look at the integer $\operatorname{deg}\left(\frac{u}{|u|}, \gamma_{r_{1}}\right)$, the degree of $\frac{u}{|u|}: \gamma_{r_{1}} \rightarrow S^{1}$. Say it is 1000 .

Inside $B_{r_{1}}(0)$ we can choose $\gamma_{r_{2}}=\partial B_{r_{2}}(0)$ lying in $D^{2} \backslash \pi$ (Sing $C$ ) so that in the annulus $B_{r_{1}}(0) \backslash \overline{B_{r_{2}}(0)}$ there are $1001+k$ of the points $z_{j}$. This is possible by the contradiction assumption of actually having a sequence converging to 0 . Around each such $z_{l}$ take an oriented loop $\gamma_{l}$ which encloses exactly one of them. We can of course ensure that each $\gamma_{l}$ lies in the annulus and does not meet $\pi(\operatorname{Sing} C)$. We know from (i) that $\operatorname{deg}\left(\frac{u}{|u|}, \gamma_{l}\right) \geq 1$ for all $l$.

By homotopy, since $\frac{u}{|u|}$ is continuous on $D^{2} \backslash \pi(\operatorname{Sing} C)=\{u \neq 0\}$, we have

$$
\operatorname{deg}\left(\frac{u}{|u|}, \gamma_{r_{1}}\right)=\operatorname{deg}\left(\frac{u}{|u|}, \gamma_{r_{2}}\right)+\Sigma_{l} \operatorname{deg}\left(\frac{u}{|u|}, \gamma_{l}\right) \text {, }
$$

where the summation is taken over the $1001+k$ loops $\gamma_{l}$ in the annulus. Each term in this summation is $\geq 1$. From this we get $\operatorname{deg}\left(\frac{u}{|u|}, \gamma_{r_{2}}\right) \leq k-1$, contradicting (ii).

This is also the idea in [21]. In that work, the function $u$ is defined by observing the relative difference of points having the same projection on $D^{2}$ : this is naturally an element of $\mathbb{C}$ in that case. 
But for our Special Legendrian, the relative difference naturally lives in $\mathbb{C} \times \mathbb{R}$ (see Figure 7.1), and there is no notion of degree for a function $u: D^{2} \rightarrow \mathbb{C} \times \mathbb{R}$, therefore we need to change the setting. We will introduce a new space, modelled on the product of the 2-dimensional current with $\overline{\mathbb{R}}$ and define a function $u$ from this product into $\mathbb{C} \times \mathbb{R}$; this function mimics the relative difference and that allows a homological argument.

Close enough to each isolated singularity, the $\mathbb{C}$-component of the relative difference encloses all the topological information and we can neglect the $\mathbb{R}$-component (see Lemmas 7.5 and 7.6). Unfortunately this is only possible very close to each isolated singularity, and we need to take care also of the $\mathbb{R}$-component when we seek a global estimate from below (obtained in Lemma 7.8) analogous to the one in (ii), whence the somewhat curious choice of $u$ and of its domain.

ACKNOWLEDGEMENTS. The authors are very grateful to Gang Tian for having suggested this problem to them and for very fruitful discussions.

\section{Preliminaries: the construction of positively intersecting foliations}

In this section we are going to construct in a generic way a smooth 3-surface $\Sigma$ in $S^{5}$ with the property that, anytime $\Sigma$ intersects a Special Legendrian $L$ transversally, this intersection is positive, i.e., the orientation of $T_{p} L \wedge T_{p} \Sigma$ agrees with that of $T_{p} S^{5}$ ( $S^{5}$ being oriented according to the outward normal). Then we will construct foliations made with families of 3-surfaces of this kind.

Contact structure. Now we recall some basic facts on the geometry of the contact structure associated to the Special Legendrian calibration in $S^{5}$, see [11] for more details.

$S^{5}$ inherits from the symplectic manifold $\left(\mathbb{C}^{3}, \sum_{i=1}^{3} d z^{i} \wedge d \bar{z}^{i}\right)$ the contact structure given by the form

$$
\gamma:=\mathcal{E}^{*} \iota_{N}\left(\sum_{i=1}^{3} d z^{i} \wedge d \bar{z}^{i}\right) .
$$

This is a 1 -form with the contact property saying that $\gamma \wedge(d \gamma)^{2} \neq 0$ everywhere; the associated distribution of hyperplanes is $\operatorname{ker}(\gamma(p)) \subset T_{p} S^{5}$. In the sequel the hyperplane of the distribution at $p$ will be denoted by $H_{p}^{4}$, where $H$ stands for horizontal ${ }^{4}$. The condition on $\gamma$ is equivalent to the non-integrability of this distri-

4 This is nothing else but the universal horizontal connection associated to the Hopf projection $S^{5} \rightarrow \mathbb{C P}^{2}$ sending $\left(z_{1}, z_{2}, z_{3}\right) \rightarrow\left[z_{1}, z_{2}, z_{3}\right]$. The fibers $e^{i \theta} p, \theta \in[0,2 \pi]$ and $p \in S^{5}$, are great circles in $S^{5}$ and the hyperplanes $H_{p}^{4}$ of the horizontal distribution are everywhere orthogonal to the fibers. This structure is $S U(3)$-invariant. 
bution, i.e. it is impossible (even locally) to find a 4-surface in $S^{5}$ which is everywhere tangent to the $H^{4}$. The vectors $v$ orthogonal to $H^{4}$ are called vertical; they are everywhere tangent to the Hopf fibers $e^{i \theta}\left(z_{1}, z_{2}, z_{3}\right) \subset S^{5}$.

Special Legendrians are tangent to the horizontal distribution. The Special Legendrian calibration $\omega$ has the property that any calibrated 2-plane in $T S^{5}$ must be contained in $H^{4}$. Therefore, Special Legendrian submanifolds are everywhere tangent to the horizontal distribution and they are a particular case of the so called Legendrian curves, which are the maximal dimensional integral submanifolds of the contact distribution. We can shortly justify this as follows: recall that $\omega$ and the horizontal distribution are invariant under the action of $S U(3)$. At the point $(1,0,0) \in$ $S^{5}$ the Special Legendrian semi-calibration is easily ${ }^{5}$ computed: $\omega_{(1,0,0)}=d x^{2} \wedge$ $d x^{3}-d y^{2} \wedge d y^{3}$. Then if a unit simple 2-vector in $T_{(1,0,0)} S^{5}$ is calibrated, it must lie in the 4-plane spanned by the coordinates $x_{2}, y_{2}, x_{3}, y_{3}$, which is the horizontal hyperplane $H_{(1,0,0)}^{4}$ orthogonal to the Hopf fiber $e^{i \theta}(1,0,0)$. The $S U(3)$-invariance of $\omega$ and of $\left\{H^{4}\right\}$ implies that, at all points on the sphere, Special Legendrians are tangent to the horizontal distribution.

$\boldsymbol{J}$-structure and $\boldsymbol{J}$-invariance. We introduce now a further structure: on each hyperplane $H_{p}^{4}, \omega$ restricts to a non-degenerate 2-form, so we get a symplectic structure and we can define the (unique) linear map

$$
J_{p}: H_{p}^{4} \rightarrow H_{p}^{4}
$$

characterized by the properties that $J_{p}^{2}=-I d$ and, for $v, w \in H_{p}^{4}$,

$$
\omega(p)(v, w)=\omega(p)\left(J_{p} v, J_{p} w\right), \quad\langle v, w\rangle_{T_{p} S^{5}}=\omega(p)\left(v, J_{p} w\right) .
$$

This is a standard construction from symplectic geometry and the uniqueness of the $J_{p}$ at each point implies that we get a smooth endomorphism of the horizontal bundle; in our case the setting is simple enough to allow an explicit expression of $J_{p}$ in coordinates, as follows.

$\omega_{(1,0,0)}=d x^{2} \wedge d x^{3}-d y^{2} \wedge d y^{3}$ and recall that $H_{(1,0,0)}^{4}$ is spanned by the coordinates $x_{2}, y_{2}, x_{3}, y_{3}$. Then choose

$$
J_{(1,0,0)}:=\left\{\begin{aligned}
\frac{\partial}{\partial x_{2}} & \rightarrow \frac{\partial}{\partial x_{3}} \\
\frac{\partial}{\partial y_{2}} & \rightarrow-\frac{\partial}{\partial y_{3}} .
\end{aligned}\right.
$$

The conditions in (2.1) hold true at this point.

For any $p \in S^{5}$, take $g \in S U(3) / S U(2)$ sending $p$ to $(1,0,0)$. The $S U(2)$ in the quotient is the stabilizer of $H_{(1,0,0)}^{4}$. This stabilizer leaves $J_{(1,0,0)}$ invariant (any

${ }^{5}$ Recall that we are using standard coordinates $z_{j}=x_{j}+i y_{j}, j=1,2,3$ on $\mathbb{C}^{3}$. 
element of $S U(2)$ commutes with $\left.J_{(1,0,0)}\right)$ and we can define, for $v \in H_{p}^{4}$,

$$
J_{p}(v):=d g^{-1}\left(J_{(1,0,0)}(d g(v))\right) .
$$

Thus we get a smooth $J$-structure on the horizontal bundle.

From the properties in (2.1), if a simple unit 2-vector $v \wedge w$ in $H_{p}^{4}$ is calibrated by $\omega$, then

$$
1=\omega_{p}(v, w)=\omega_{p}\left(J_{p} v, J_{p} w\right)=\left\langle J_{p} v, w\right\rangle_{T_{p} S^{5}}
$$

so

$v \wedge w$ is a Special Legendrian plane $\Leftrightarrow J_{p}(v \wedge w):=J_{p} v \wedge J_{p} w=v \wedge w$, i.e.

Proposition 2.1. A 2-plane in $T_{p} S^{5}$ is Special Legendrian if and only if it lies in $H_{p}^{4}$ (horizontal for the Hopf connection) and it is $J_{p}$-invariant for the $J$-structure above.

Since all the above introduced objects are invariant under the action of $S U(3)$, we can afford to work at a given point of $S^{5}$; from now on we will focus on a neighbourhood of the point $(1,0,0) \in S^{5}$, where we are using the complex coordinates $\left(z_{1}, z_{2}, z_{3}\right)=\left(x_{1}, y_{1}, x_{2}, y_{2}, x_{3}, y_{3}\right)$ of $\mathbb{C}^{3}$.

Positive 3-surface. We are now ready for the construction of a 3-surface with the property of positive intersection.

Oriented $m$-planes in $\mathbb{C}^{3}$ will be identified with unit simple $m$-vectors in $\mathbb{C}^{3}$. In particular, $T S^{5}$ is oriented so that $T S^{5} \wedge \frac{\partial}{\partial r}=\mathbb{C}^{3}$.

Writing down the Special Lagrangian calibration explicitly

$\Omega=d x^{1} \wedge d x^{2} \wedge d x^{3}-d x^{1} \wedge d y^{2} \wedge d y^{3}-d y^{1} \wedge d x^{2} \wedge d y^{3}-d y^{1} \wedge d y^{2} \wedge d x^{3}$,

it is straightforward to see that

$$
\mathcal{L}_{0}=\frac{\partial}{\partial x_{1}} \wedge \frac{\partial}{\partial x_{2}} \wedge \frac{\partial}{\partial x_{3}}
$$

is a Special Lagrangian 3-plane passing through the origin of $\mathbb{C}^{3}$ and through the point $(1,0,0)$. We now consider, for a small positive $\varepsilon$, the following family $\left\{\mathcal{L}_{\theta}\right\}_{\theta \in(-\varepsilon, \varepsilon)}$ of Special Lagrangian planes, where $\left\{\left(e^{i \theta}, 0,0\right)\right\}_{\theta \in(-\varepsilon, \varepsilon)}$ is the fiber containing $(1,0,0)$ and $\mathcal{L}_{\theta}$ goes through the point $\left(e^{i \theta}, 0,0\right)$ :

$$
\begin{aligned}
\mathcal{L}_{\theta} & =\left(\begin{array}{ccc}
e^{i \theta} & 0 & 0 \\
0 & e^{-i \theta} & 0 \\
0 & 0 & 1
\end{array}\right)_{*} \mathcal{L}_{0} \\
& =\left(\cos \theta \frac{\partial}{\partial x_{1}}+\sin \theta \frac{\partial}{\partial y_{1}}\right) \wedge\left(\cos \theta \frac{\partial}{\partial x_{2}}-\sin \theta \frac{\partial}{\partial y_{2}}\right) \wedge \frac{\partial}{\partial x_{3}},
\end{aligned}
$$


which is Special Lagrangian since it has been obtained by pushing forward $\mathcal{L}_{0}$ by an element in $S U(3)$.

We introduce the 4-surface $\Sigma^{4}$ in $\mathbb{C}^{3}$ obtained by attaching the $\mathcal{L}_{\theta}$-planes along the fiber $\left\{\left(e^{i \theta}, 0,0\right)\right\}_{\theta \in(-\varepsilon, \varepsilon)}$ : this 4 -surface can be expressed as

$$
\Sigma^{4}=\left(a e^{i \theta}, b e^{-i \theta}, c\right)
$$

parametrized with $(a, b, c) \in \mathbb{R}^{3} \backslash\{0\}, \quad \theta \in(-\varepsilon, \varepsilon)$. Then define

$$
\Sigma=\Sigma^{4} \cap S^{5} \text {. }
$$

As stated in the coming Lemma 2.2, this 3-surface has the desired property of intersecting Special Legendrians positively.

We can make the equivalent construction starting from the form $\omega$ restricted to the fiber $\left\{\left(e^{i \theta}, 0,0\right)\right\}_{\theta \in(-\varepsilon, \varepsilon)}$, namely

$$
\omega=\cos \theta\left(d x^{2} \wedge d x^{3}-d y^{2} \wedge d y^{3}\right)+\sin \theta\left(-d x^{2} \wedge d y^{3}-d y^{2} \wedge d x^{3}\right),
$$

and explicitly writing down the J-structure on $H_{\left(e^{i \theta}, 0,0\right)}^{4}$ introduced above. On $H_{\left(e^{i \theta}, 0,0\right)}^{4}$ we can use coordinates $\left(x_{2}, y_{2}, x_{3}, y_{3}\right)$ since $H^{4} \wedge v=T S^{5}, T S^{5} \wedge \frac{\partial}{\partial r}=$ $\mathbb{C}^{3}$ and $v=i \frac{\partial}{\partial r}$, so $H^{4}=\frac{\partial}{\partial x_{2}} \wedge \frac{\partial}{\partial y_{2}} \wedge \frac{\partial}{\partial x_{3}} \wedge \frac{\partial}{\partial y_{3}}$.

$$
J_{\theta}=J_{\left(e^{i \theta}, 0,0\right)}:=\left\{\begin{array}{l}
\frac{\partial}{\partial x_{2}} \rightarrow \cos \theta \frac{\partial}{\partial x_{3}}-\sin \theta \frac{\partial}{\partial y_{3}} \\
\frac{\partial}{\partial y_{2}} \rightarrow-\cos \theta \frac{\partial}{\partial y_{3}}-\sin \theta \frac{\partial}{\partial x_{3}} \\
\frac{\partial}{\partial x_{3}} \rightarrow-\cos \theta \frac{\partial}{\partial x_{2}}+\sin \theta \frac{\partial}{\partial y_{2}} \\
\frac{\partial}{\partial y_{3}} \rightarrow \cos \theta \frac{\partial}{\partial y_{3}}+\sin \theta \frac{\partial}{\partial x_{2}} .
\end{array}\right.
$$

So $J_{\theta}$ is represented by the matrix $J_{0} A_{\theta}$, where 6

$$
J_{0}=\left(\begin{array}{rrrr}
0 & 0 & -1 & 0 \\
0 & 0 & 0 & 1 \\
1 & 0 & 0 & 0 \\
0 & -1 & 0 & 0
\end{array}\right), A_{\theta}=\left(\begin{array}{cccc}
\cos \theta & \sin \theta & 0 & 0 \\
-\sin \theta & \cos \theta & 0 & 0 \\
0 & 0 & \cos \theta & -\sin \theta \\
0 & 0 & \sin \theta & \cos \theta
\end{array}\right)
$$

${ }^{6}$ In complex notation, looking at $H_{\left(e^{i \theta}, 0,0\right)}^{4}$ as $\mathbb{C}_{z_{2}, z_{3}}^{2}$, we can write

$$
A_{\theta}=\left(\begin{array}{cc}
e^{i \theta} & 0 \\
0 & e^{-i \theta}
\end{array}\right) .
$$


If $v \wedge w$ is a $J$-invariant 2-plane in $H_{0}^{4}$, with $w=J_{0} v$, then $A_{\theta}^{-1} v \wedge w$ is $J_{\theta}$ invariant, in fact $J_{\theta}\left(A_{\theta}^{-1} v\right)=J_{0} A_{\theta} A_{\theta}^{-1} v=J_{0} v=w$. Take the geodesic 2-sphere $L_{0}$ tangent to the $J_{0}$-holomorphic plane

$$
\frac{\partial}{\partial x_{2}} \wedge \frac{\partial}{\partial x_{3}} .
$$

This Special Legendrian 2-sphere $L_{0}$ coincides with $\mathcal{L}_{0} \cap S^{5}$ introduced above. The 2-plane

$$
A_{\theta}^{-1} \frac{\partial}{\partial x_{2}} \wedge \frac{\partial}{\partial x_{3}}=\left(\cos \theta \frac{\partial}{\partial x_{2}}-\sin \theta \frac{\partial}{\partial y_{2}}\right) \wedge \frac{\partial}{\partial x_{3}}
$$

is therefore $J_{\theta}$ holomorphic and the geodesic 2-sphere tangent to it is $\mathcal{L}_{\theta} \cap S^{5}$. $\Sigma$ is the 3-surface obtained from the union of those Special Legendrian spheres as $\theta \in(-\varepsilon, \varepsilon)$.

Lemma 2.2. There is an $\varepsilon_{0}>0$ small enough such that for any $\varepsilon<\varepsilon_{0}$ the following holds:

let $S$ be any Special Legendrian current in $B_{\varepsilon}(1,0,0) \subset S^{5}$; then, at any point $p$ where $T_{p} S$ is defined and transversal to $T_{p} \Sigma, S$ and $\Sigma$ intersect each other in a positive way, i.e.

$$
T_{p} S \wedge T_{p} \Sigma=T_{p} S^{5} .
$$

Proof of Lemma 2.2.

$$
T_{\left(e^{i \theta}, 0,0\right)} \Sigma=A_{\theta}^{-1} \frac{\partial}{\partial x_{2}} \wedge \frac{\partial}{\partial x_{3}} \wedge v_{\theta}
$$

so, along the fiber, the tangent space to $\Sigma$ is spanned by two vectors $l^{1}, l^{2}$ such that $l^{1} \wedge l^{2}$ is Special Legendrian and by the vertical vector $v_{\theta}$. At any other point $p$ of $\Sigma$, the tangent space always contains two directions $l_{p}^{1}, l_{p}^{2}$ such that $l_{p}^{1} \wedge l_{p}^{2}$ is Special Legendrian (from the construction of $\Sigma$ ). The third vector $w$, orthogonal to these two and such that $l_{p}^{1} \wedge l_{p}^{2} \wedge w=T \Sigma$, drifts from the vertical direction as the point moves away from the fiber, but by continuity, for a small neighbourhood $B_{\varepsilon}(1,0,0)$, we still have that

$$
H_{p}^{4} \wedge w_{p}=T_{p} S^{5}
$$

On the other hand, it is a general fact that, given a 4-plane with a J-structure, two transversal $J$-invariant planes always intersect positively. Therefore

$$
T_{p} S \wedge l_{p}^{1} \wedge l_{p}^{2}=H_{p}^{4}
$$

at any point $p$, so

$$
T_{p} S \wedge T_{p} \Sigma=T_{p} S \wedge\left(l_{p}^{1} \wedge l_{p}^{2} \wedge w_{p}\right)=\left(T_{p} S \wedge l_{p}^{1} \wedge l_{p}^{2}\right) \wedge w_{p}=T_{p} S^{5} .
$$


First parallel foliation. Now we are going to exhibit a 2-parameter family of 3surfaces that foliate $B_{\varepsilon}(1,0,0)$ and have the property of positive intersection. Consider the Special Legendrian 2-sphere

$$
L=\left(-\frac{\partial}{\partial x_{1}} \wedge \frac{\partial}{\partial y_{2}} \wedge \frac{\partial}{\partial y_{3}}\right) \cap S^{5} .
$$

This is going to be the space of parameters. Consider $S O(3)$ and let it act on the 3 -space $-\frac{\partial}{\partial x_{1}} \wedge \frac{\partial}{\partial y_{2}} \wedge \frac{\partial}{\partial y_{3}}$. We are only interested in the subgroup of rotations having axis in the plane $\frac{\partial}{\partial y_{2}} \wedge \frac{\partial}{\partial y_{3}}$. This subgroup is isomorphic to $S O(3) / S$, where $S$ is the stabilizer of a point, in our case the point $(1,0,0) \in-\frac{\partial}{\partial x_{1}} \wedge \frac{\partial}{\partial y_{2}} \wedge \frac{\partial}{\partial y_{3}}$. Thus the rotations in this subgroup can be parametrized over the points of $L=$ $\left(-\frac{\partial}{\partial x_{1}} \wedge \frac{\partial}{\partial y_{2}} \wedge \frac{\partial}{\partial y_{3}}\right) \cap S^{5}$ and we will write $A_{q}$ for the rotation sending $(1,0,0)$ to $q \in L$. We extend $A_{q}$ to a rotation of the whole $S^{5}$ by letting it act diagonally on $\mathbb{R}^{3} \oplus \mathbb{R}^{3}=\mathbb{C}^{3}$. Then define

$$
\Sigma_{q}=A_{q}(\Sigma),
$$

for $q \in L$. Since $A_{q} \in S U(3)$, Special Legendrian spheres are invariant and $A_{q}\left(e^{i \theta}(1,0,0)\right)=e^{i \theta} A_{q}((1,0,0))=e^{i \theta} q$, so the fiber through $(1,0,0)$ is sent into the fiber through $q$. Therefore, for a fixed $q, \Sigma_{q}$ is a 3-surface of the same type as $\Sigma$, that is, it contains the fiber through $q$ and is made of the union of Special Legendrian spheres smoothly attached along the fiber. By the $S U$ (3)-invariance of $\omega$, from Lemma 2.2 we get that $\Sigma_{q}$ has the property of intersecting positively any transversal Special Legendrian $S$.

For the sequel define $L_{\varepsilon}=L \cap B_{\varepsilon}(1,0,0)$.

Lemma 2.3. The 3-surfaces $\Sigma_{q}$, as $q \in L_{\varepsilon}$, foliate a neighbourhood of $(1,0,0)$ in $S^{5}$.

Proof of Lemma 2.3. Parametrize $L_{\varepsilon}$ with normal coordinates $(s, t)$, with $\frac{\partial}{\partial s}=\frac{\partial}{\partial y_{2}}$, $\frac{\partial}{\partial t}=-\frac{\partial}{\partial y_{3}}$ and $\Sigma=\Sigma_{0}$ with $(a, b, c, \theta) \in\left(S^{2} \cap B_{\varepsilon}(1,0,0)\right) \times(-\varepsilon, \varepsilon)$, with $(a, b, c) \in S^{2} \subset \mathbb{R}^{3}=\frac{\partial}{\partial x_{1}} \wedge \frac{\partial}{\partial x_{2}} \wedge \frac{\partial}{\partial x_{3}}$ and $\theta \in(-\varepsilon, \varepsilon)$ as done during the construction (we set $a=\left(1-b^{2}-c^{2}\right)^{1 / 2}$ ). Consider the function $\psi: \Sigma \times L_{\varepsilon} \rightarrow S^{5}$ defined as

$$
\psi(p, q)=A_{q}(p)
$$

for $p=(b, c, \theta) \in \Sigma, q=(s, t) \in L_{\varepsilon}$. Analysing the action of the differential $d \psi$ on the basis vectors at $(0,0) \in \Sigma \times L_{\varepsilon}$ we get:

$$
\frac{\partial \psi}{\partial b}=\frac{\partial}{\partial x_{2}}, \frac{\partial \psi}{\partial c}=\frac{\partial}{\partial x_{3}}, \frac{\partial \psi}{\partial \theta}=\frac{\partial}{\partial y_{1}}, \frac{\partial \psi}{\partial s}=\frac{\partial}{\partial y_{2}}, \frac{\partial \psi}{\partial t}=-\frac{\partial}{\partial y_{3}} .
$$

So the Jacobian determinant at 0 is 1 and $\psi$ is a diffeomorphism in some neighbourhood of $(1,0,0)$ where we can introduce the new set of coordinates $(b, c, \theta, s, t)$. Therefore, the family $\left\{\Sigma_{s, t}\right\}_{(s, t) \in L}$ foliates an open set that we can assume to be $\psi\left(\Sigma \times L_{\varepsilon}\right)$ if both $\Sigma$ and $L_{\varepsilon}$ were taken small enough. 
Coordinates induced by the first parallel foliation. Recall that, in each $H_{p}^{4}$ we are interested in the possible calibrated 2-planes, which, as shown above, must be $J_{p}$-invariant. The set of these 2-planes is parametrized by the complex lines in $\mathbb{C}^{2}$ and is therefore diffeomorphic to $\mathbb{C P}^{1}$. We are often going to identify $H^{4}$ with $\mathbb{C}^{2}$ (respectively $\mathbb{C P}^{1}$, if we are interested in the complex lines) with the following coordinates: on $H_{(1,0,0)}^{4}$ we set $H_{(1,0,0)}^{4}=T L \oplus T\left(L_{0}\right)=\mathbb{C}_{s+i t} \oplus \mathbb{C}_{b+i c}$, where $L, L_{0}$ are the Special Legendrians introduced above; $T L, T L_{0}$ are $\mathbb{C}$-orthogonal complex lines in $H_{(1,0,0)}^{4}, T L=\frac{\partial}{\partial s} \wedge \frac{\partial}{\partial t}$ and $T L_{0}=\frac{\partial}{\partial b} \wedge \frac{\partial}{\partial c}$. Then the complex line $L$ will be represented by $[1,0]$ in $\mathbb{C P}^{1}$ and $L_{0}$ by $[0,1]$. Extend these coordinates to the other hyperplanes $H^{4}$ as follows: at any $H_{p}^{4}$ we have that, for the unique $\Sigma$ containing $p$ :

$$
T_{p} L=[1,0], \quad T_{p} \Sigma \cap H_{p}^{4}=[0,1] .
$$

Families of parallel foliations. We will often need to use not only the foliation constructed, but a family of foliations. Keeping as base coordinates the coordinates that we just introduced, we can perform a similar construction. The foliation we constructed is parametrized by $q \in L$ with the property that $T_{q} \Sigma_{q} \cap H_{q}^{4}=[0,1] \in$ $\mathbb{C P}^{1}$. For $X$ in a neighbourhood of $[0,1] \in \mathbb{C P}^{1}$, e.g. $\left\{X=[Z, W] \in \mathbb{C P}^{1}\right.$, $|Z| \leq|W|\}$, we start from the 3 -surface $\Sigma_{0}^{X}$ built as follows: the Special Legendrian spheres that we attach to the fiber should have tangent planes in the direction $X \in$ $\mathbb{C P}^{1}$. Then, for any such fixed $X$, we still have a foliation of a neighbourhood of $(1,0,0)$, parametrized on $L$ and made of the 3 -surfaces

$$
\Sigma_{q}^{X}:=A_{q}\left(\Sigma_{0}^{X}\right), \quad q \in L .
$$

We will refer to $\Sigma_{q}^{X}$ as to the 3-surface born at $q$ in the direction $X$. The original surfaces we built will be denoted $\Sigma^{[0,1]}$. By the $S U(3)$-invariance of $\omega$, from Lemma 2.2 we get the positiveness property for $\Sigma_{q}^{X}$ :

Corollary 2.4. For any $q, \Sigma_{q}^{X}$ has the property of intersecting positively any transversal Special Legendrian $S$, i.e. at any point $p$ where $T_{p} S$ is defined and transversal to $T_{p} \Sigma_{q}^{X}$,

$$
T_{p} S \wedge T_{p} \Sigma_{q}^{X}=T_{p} S^{5}
$$

For a fixed $X$, a parallel foliation $\left\{\Sigma_{p}^{X}\right\}$ (as $p$ ranges over $L_{\varepsilon}$ ) gives rise in a neighbourhood of $(1,0,0)$ to a system of five real coordinates. The adjective parallel is reminiscent of this resemblance to a cartesian system of coordinates in the chosen neighbourhood. There are several reasons why we produced parallel foliations keeping freedom on the "direction" $X$; they will be clear later on.

Families of polar foliations. So far we have been dealing with "parallel" foliations. We turn now to "polar" foliations ${ }^{7}$.

7 The term polar is used as reminiscent of the standard polar coordinates in the plane. 
Notice that, a point in $L$ being fixed, say 0 , we have that, as $X$ runs over a neighbourhood of $[0,1] \in \mathbb{C P}^{1}$, the family $\left\{\Sigma_{0}^{X}\right\}$ foliates a conic neighbourhood of $\Sigma_{0}^{[0,1]}$. Observe that the rotations in $S O(3) \subset S U$ (3) fixing the fiber through $q \in S^{5}$ have for differentials exactly the rotations in $S U$ (2) on $H_{q}^{4}$. Denoting $R_{X, Y}$ the rotation whose differential sends $X$ to $Y \in H_{q}^{4}$, we have $R_{X, Y}\left(\Sigma_{q}^{X}\right)=\Sigma_{q}^{Y}$.

Lemma 2.5. With the above notations, let $U$ be a small enough neighbourhood of $Y \in \mathbb{C P}^{1}$ and consider $\Sigma_{q}^{Y}$ for some point $q$. Let $L^{Y} \subset \Sigma_{q}^{Y}$ be the Special Legendrian 2-sphere tangent to $Y$ at $q$. Then

$$
\left(\cup_{X \in U} \Sigma_{q}^{X}\right)-\left\{e^{i \theta} q\right\}
$$

is a neighbourhood of $L^{Y}-\{q\}$.

Proof of Lemma 2.5. Introduce the function $\psi: \Sigma \times U \rightarrow S^{5}$ sending $(p, X), p \in$ $\Sigma=\Sigma_{q}^{Y}, X \in U$, to the point $R_{Y, X}(p) \in \Sigma_{q}^{X}$. Observe that, in a neighbourhood of $(1,0,0)$, the differential of $\psi$ is different from zero except at the points of the Hopf fiber through $(1,0,0)$. Indeed, on this fiber, $d \psi$ restricted to the 3 -space $T \Sigma$ has rank 3 and $T \Sigma \cong Y_{1} \wedge Y_{2} \wedge v$, with $Y_{1} \wedge Y_{2}$ the 2-plane in $\mathbb{C}^{2}$ represented by $Y$. At any point $F$ among these, $d \psi$ is zero on the tangent space to $U$ at $Y$, since the image $\psi(F, X)$ is constantly equal to $F$ for any $X$. For any fixed point $p$ not on the fiber and for $X$ on a curve in $U$ through $Y, \psi(p, X)$ is a curve transversal to $\Sigma_{p}^{Y}$, since we are moving $p$ by the rotation $R_{Y, X}$. Therefore the differential $d \psi(p, Y)$ has rank 2 when restricted to the tangent to $U$ at $Y$, while on the complementary 3 -space $d \psi$ still has rank 3 by smoothness. Therefore we get the desired result.

Remark 2.6. We remark here that a 3 -surface $\Sigma$ of the type just exhibited above, is foliated by Special Legendrian spheres, so the Special Legendrian structure restricted to $\Sigma$ is integrable; a Special Legendrian integral cycle contained in such a $\Sigma$ must locally be one of these spheres.

Remark 2.7. With the above notations, $L_{0}+L$ is a Special Legendrian cycle with isolated singularities at the points $(1,0,0)$ and $(-1,0,0)$. This example shows that our regularity result is optimal. The reader may consult [11] for further explicit examples of Special Legendrian surfaces.

\section{Tools from intersection theory}

In this section we recall some basic facts about the blowing-up of the current at a point and about the Kronecker intersection index (for the related issues in geometric measure theory we refer to [8]); then we show that this index is preserved when we send a blown-up sequence to the limit.

Let $C$ be the Special Legendrian cycle that we are studying. The blow-up analysis of the current $C$ around a point $x_{0}$ is performed as follows: consider a 
dilation of $C$ around $x_{0}$ of factor $r$ which, in normal coordinates around $x_{0}$, is expressed by the push-forward of $C$ under the action of the map $\frac{x-x_{0}}{r}$ :

$$
C_{x_{0}, r}(\psi)=\left[\left(\frac{x-x_{0}}{r}\right)_{*} C\right](\psi)=C\left(\left(\frac{x-x_{0}}{r}\right)^{*} \psi\right) .
$$

From [16] or [19] we have the monotonicity formula ${ }^{8}$ which states that, for any $x_{0}$, the function mass ratio, i.e.

$$
\frac{M\left(C\left\llcorner B_{r}\left(x_{0}\right)\right)\right.}{r^{2}},
$$

is monotonically non-increasing as $r \downarrow 0$, therefore ${ }^{9}$ the limit

$$
\theta(x):=\lim _{r \rightarrow 0} \frac{M\left(C\left\llcorner B_{r}(x)\right)\right.}{\pi r^{2}}
$$

exists for any point $x \in S^{5}$. This limit coincides (a.e.) with the multiplicity $\theta$ assigned in the definition ${ }^{10}$ of integer cycle, whence the use of the same notation. We can therefore speak of the multiplicity function $\theta$ as a (everywhere) well-defined function on $\mathcal{C}$.

We recall the definitions of weak-convergence and flat-convergence for a sequence $T_{n}$ of currents in $\mathcal{R}_{m}$ to $T \in \mathcal{R}_{m}$. We remark, however, that the notions of weak-convergence and flat-convergence turn out to be equivalent for integral currents of equibounded mass and boundary mass (as it is in our case), see [19, 31.2] or [8, page 516$]$.

We say that $T_{n} \rightarrow T$ weakly when we look at the dual pairing with $m$-forms, i.e. if $T_{n}(\psi) \rightarrow T(\psi)$ for any smooth and compactly supported $m$-form $\psi$.

$T_{n} \rightarrow T$ in the Flat-norm if the quantity $\mathcal{F}\left(T-T_{n}\right):=\inf \{M(A)+M(B):$ $T-T_{n}=A+\partial B, A \in \mathcal{R}_{m}, B \in \mathcal{R}_{m+1}$ \} goes to 0 as $n \rightarrow \infty$.

The fact that $\frac{M\left(C L_{B_{r}}\left(x_{0}\right)\right)}{r^{2}}$ is monotonically non-increasing as $r \downarrow 0$ gives that, for $r \leq r_{0}$ (for a small enough $r_{0}$ ), we are dealing with a family of currents $\left\{C_{x_{0}, r}\right\}$ which are boundaryless and locally equibounded in mass; by Federer-Fleming's compactness theorem ${ }^{11}$, there exist a sequence $r_{n} \rightarrow 0$ and a rectifiable boundaryless current $C_{\infty}$ such that

$$
C_{x_{0}, r_{n}} \rightarrow C_{\infty} \text { in Flat-norm. }
$$

8 This formula is proved in [16] for semi-calibrated currents and in [19] for currents of vanishing mean curvature; both cases apply here.

9 To be precise, due to the fact that the metric is not flat, the mass ratio is almost monotone, i.e. $\frac{M\left(C\left\llcorner B_{r}\left(x_{0}\right)\right)\right.}{r^{2}}=R(r)+O(r)$ for a function $R$ which is monotonically non-increasing as $r \downarrow 0$ and tends to the multiplicity at $x_{0}$ as $r \downarrow 0$, and a function $O(r)$ which is infinitesimal. The additional infinitesimal term $O(r)$ does not affect the analysis we need to perform.

10 The multiplicity $\theta$ can be assumed to be positive by choosing the right orientation for the approximate tangent planes to the current.

${ }^{11}$ See [8, page 141]. 
$C_{\infty}$ turns out to be a cone (a so called tangent cone to $C$ at $x_{0}$ ) with density at the origin the same as the density of $C$ at $x_{0}$ and calibrated by $\omega_{x_{0}}$ (see $[10$, Section II.5]); being $J_{x_{0}}$-holomorphic, this cone must be a sum of $J_{x_{0}}$-holomorphic planes, so $C_{\infty}=\bigoplus_{i=1}^{Q} D_{i}$, where the $D_{i}$ 's are (possibly coinciding) Special Legendrian disks. In particular, the multiplicity (the limit of the mass ratio) $\theta$ is everywhere $\mathbb{N}$-valued in our case.

An important question for regularity issues is to know whether this tangent cone is unique or not, or, in other words, if $C_{\infty}$ is independent of the chosen $\left\{r_{n}\right\}$ : the answer happens to be positive in our situation. We are going to give a selfcontained proof of it in the next section (Theorem 4.5) based on the tools from this section.

What kind of geometric information can we draw from the existence of a tangent cone? The following lemma shows that, considering a blown-up sequence $C_{x_{0}, r_{n}}$ tending to one possible tangent cone $C_{\infty}$, we can fix a conic neighbourhood of $C_{\infty}$, as narrow as we want, and if we neglect a ball around zero of any radius $R<1$ the restrictions of $C_{x_{0}, r_{n}}$ to the annulus $B_{1} \backslash B_{R}$ are supported in the chosen conic neighbourhood for $n$ large enough ${ }^{12}$.

Remark 3.1. It is a standard fact that two distinct sequences $C_{x_{0}, r_{n}}$ and $C_{x_{0}, \rho_{n}}$ must tend to the same tangent cone if $a \leq \frac{r_{n}}{\rho_{n}} \leq b$ for some positive numbers $a$ and $b$. See [14].

Lemma 3.2. Let $C$ be a Special Legendrian cycle with $x_{0} \in C$ and let $0<R<1$. With the above notations, let $\rho_{n} \rightarrow 0$ be such that $C_{x_{0}, \rho_{n}} \rightarrow C_{\infty}=\oplus_{i=1}^{Q} D_{i}$. Denote by $A_{R}$ the annulus $\left\{x \in B_{1}(0),|x| \geq R\right\}$ and by $E_{\varepsilon}$ the set $\left\{x \in B_{1}(0)\right.$, $\left.\operatorname{dist}\left(x, C_{\infty}\right)<\varepsilon|x|\right\}$. Then, for any $\varepsilon>0$, there is $n_{0} \in \mathbb{N}$ large enough such that

$$
\operatorname{spt}_{x_{0}, \rho_{n}} \cap A_{R} \subset E_{\varepsilon}
$$

for $n \geq n_{0}$.

Proof of Lemma 3.2. Arguing by contradiction, we assume the existence of $\varepsilon_{0}>0$ such that

$$
\forall n \exists x_{n} \in \operatorname{spt}_{x_{0}, \rho_{n}} \cap E_{\varepsilon_{0}}^{c} \cap A_{R} .
$$

Recall that the sequence $C_{x_{0}, \rho_{n}\left|x_{n}\right|}$ also converges weakly to the same tangent cone $C_{\infty}$ since $R \leq \frac{\rho_{n}\left|x_{n}\right|}{\rho_{n}} \leq 1$ (previous remark). From the monotonicity formula we have

$$
M\left(C_{x_{0}, \rho_{n}\left|x_{n}\right|}\left\llcorner B \frac{\varepsilon_{0}}{2}\left(\frac{x_{n}}{\left|x_{n}\right|}\right)\right) \geq \frac{\pi \varepsilon_{0}^{2}}{4} .\right.
$$

By compactness, modulo extraction of a subsequence, we can assume that $\frac{x_{n}}{\left|x_{n}\right|} \rightarrow$ $x_{\infty} \in \partial B_{1} \cap E_{\varepsilon_{0}}^{c}$. Then, since for $n$ large enough $B_{\frac{3 \varepsilon_{0}}{4}}\left(x_{\infty}\right) \supset B_{\frac{\varepsilon_{0}}{2}}\left(\frac{x_{n}}{\left|x_{n}\right|}\right)$, we get

$$
M\left(C_{x_{0}, \rho_{n}\left|x_{n}\right|}\left\llcorner B_{\frac{3 \varepsilon_{0}}{4}}\left(x_{\infty}\right)\right) \geq \frac{\pi \varepsilon_{0}^{2}}{4} .\right.
$$

12 Recall that $C_{x_{0}, r}$ lives in a normal chart centered at 0 . 
Recall that, from the semi-calibration property, we have

$$
M\left(C_{x_{0}, \rho_{n}\left|x_{n}\right|}\left\llcorner B_{\frac{3 \varepsilon_{0}}{4}}\left(x_{\infty}\right)\right)=\left(C_{x_{0}, \rho_{n}\left|x_{n}\right|}\left\llcorner B_{\frac{3 \varepsilon_{0}}{4}}\left(x_{\infty}\right)\right)\left({\frac{i d}{\rho_{n}\left|x_{n}\right|}}^{*} \omega\right)\right.\right.
$$

moreover

$$
\frac{i d}{\rho_{n}\left|x_{n}\right|} \stackrel{*}{C^{\infty}\left(B_{1}\right)} \omega_{0}
$$

as $n \rightarrow \infty$, where $\omega_{0}$ is the constant 2-form $\omega(0)$. Putting all together, we can write (the first equality expresses the fact that $\omega_{0}$ is a calibration for $C_{\infty}$ )

$$
\begin{aligned}
M\left(C_{\infty}\left\llcorner B_{\frac{3 \varepsilon_{0}}{4}}\left(x_{\infty}\right)\right)\right. & =\left(C_{\infty}\left\llcorner B_{\frac{3 \varepsilon_{0}}{4}}\left(x_{\infty}\right)\right)\left(\omega_{0}\right)\right. \\
& =\lim _{n}\left(C_{x_{0}, \rho_{n}\left|x_{n}\right|}\left\llcorner B_{\frac{3 \varepsilon_{0}}{4}}\left(x_{\infty}\right)\right)\left(\omega_{0}\right)\right. \\
& =\lim _{n}\left(C_{x_{0}, \rho_{n}\left|x_{n}\right|}\left\llcorner B_{\frac{3 \varepsilon_{0}}{4}}\left(x_{\infty}\right)\right)\left({\frac{i d}{\rho_{n}\left|x_{n}\right|}}^{*} \omega\right)\right. \\
& =\lim _{n} M\left(C_{x_{0}, \rho_{n}\left|x_{n}\right|}\left\llcorner B_{\frac{3 \varepsilon_{0}}{4}}\left(x_{\infty}\right)\right) \geq \frac{\pi \varepsilon_{0}^{2}}{4},\right.
\end{aligned}
$$

which contradicts the fact that $s p t C_{\infty} \cap B_{\frac{3 \varepsilon_{0}}{4}}\left(x_{\infty}\right)=\emptyset$.

We need some more tools from intersection theory. For the theory of intersection and of the Kronecker index we refer to [8, Chapter 5, Section 3.4]. We recall the definition of the index relevant to our case.

Let $f: \mathbb{R}^{5} \times \mathbb{R}^{5} \rightarrow \mathbb{R}^{5}$ be the function $f(x, y)=x-y$. The Kronecker intersection index $k(S, T)$ for two currents of complementary dimensions $S \in \mathcal{R}_{k}\left(\mathbb{R}^{5}\right), T \in \mathcal{R}_{5-k}\left(\mathbb{R}^{5}\right)$ is defined under the following conditions:

$$
\operatorname{spt} S \cap \operatorname{spt}(\partial T)=\emptyset \text { and } \operatorname{spt} T \cap \operatorname{spt}(\partial S)=\emptyset,
$$

which imply

$$
0 \notin f(\operatorname{spt}(\partial(S \times T))) .
$$

Then there is an $\varepsilon>0$ such that $B_{\varepsilon}(0) \cap f(\operatorname{spt}(\partial(S \times T)))=\emptyset$. By the constancy theorem ([8] page 130) we can define the index $k(S, T)$ as the only number such that $^{13}$

$$
f_{*}(S \times T)\left\llcorner B_{\varepsilon}(0)=k(S, T) \llbracket B_{\varepsilon}(0) \rrbracket .\right.
$$

$k(S, T)$ turns out to be an integer.

For $S, T$ as above, whenever the intersection $S \cap T$ exists (in that case, $S \cap T$ is a sum of Dirac deltas with integer weights), then $k(S, T)=(S \cap T)(1)$. In particular,

13 We are using $f_{*}$ to denote the push-forward under $f$; in [8] the notation is $f_{\sharp}$.

The brackets $\llbracket B_{\varepsilon}(0) \rrbracket$ denote the current of integration on $B_{\varepsilon}(0)$. 
when $S$ and $T$ are standard submanifolds $k(S, T)$ just counts intersections with signs as in the classical intersection theory.

In the following lemma we focus on a chosen sequence $C_{x_{0}, \rho_{n}}$ converging to a possible cone $C_{\infty}=\oplus_{i=1}^{Q} D_{i}$. For notational convenience we set $C_{n}:=$ $C_{x_{0}, \rho_{n}}\left\llcorner B_{1}(0)\right.$ and $C:=C_{\infty}\left\llcorner B_{1}(0)\right.$, always assuming to be in a normal chart with $x_{0}$ at the origin.

Lemma 3.3. Let $C_{n} \rightarrow C$ in $B_{1}$. Take $\Sigma$ to be any 3-surface such that $\Sigma \cap C \cap$ $\partial B_{1}=\emptyset$. Then, for all $n$ large enough, $k\left(C_{n}, \Sigma\right)=k(C, \Sigma)$, where $k$ is the Kronecker index just defined.

Proof of Lemma 3.3. Define $T_{n}:=C-C_{n} . T_{n} \rightarrow 0$ in the Flat-norm of $B_{1}$, so we can write $T_{n}=S_{n}+\partial R_{n}$, with $M\left(T_{n}\right)+M\left(S_{n}\right) \rightarrow 0$, where $S_{n} \in \mathcal{R}_{2}$ and $R_{n} \in \mathcal{R}_{3}$. From the hypothesis on $\Sigma$ we can choose $\varepsilon>0$ small enough to ensure that $\Sigma \cap E_{\varepsilon} \cap A_{R}=\emptyset$, where $E_{\varepsilon} \cap A_{R}=\left\{x \in B_{1},|x| \geq R, \operatorname{dist}(x, C)<\varepsilon|x|\right\}$, for some suitable $0<R<1$. For all $n$ big enough, from Lemma 3.2, we get that spt $T_{n} \cap A_{R} \subset E_{\varepsilon}$; in particular, the condition (3.2) on the boundaries of $\Sigma$ and $C$ is fulfilled and the intersection index $k\left(T_{n}, \Sigma\right)$ is well-defined.

Denote by $\tau_{a} \Sigma$, as in [8], the push-forward $\left(\tau_{a}\right)_{*}[\Sigma]$ of $\Sigma$ by the translation map $\tau_{a}$, where $a$ is a vector. The Kronecker index is invariant by homotopies keeping the boundaries condition, so we can assume that all the intersections we will deal with are well defined as integer 0-dim rectifiable currents: in fact, for a fixed $n$, the intersection $T_{n} \cap \tau_{a} \Sigma$ exists for a.e. $a$, and $n$ runs over a countable set. Obviously

$$
k\left(C_{n}-C, \Sigma\right)=k\left(S_{n}, \Sigma\right)+k\left(\partial R_{n}, \Sigma\right) ;
$$

we are going to show that both terms on the r.h.s. are zero for $n$ large enough.

From [8] we have that (the index $k$ counts the points of intersection with signs)

$$
k\left(\partial R_{n}, \Sigma\right)=\left(\partial R_{n} \cap \Sigma\right)(1) .
$$

On the other hand,

$$
\partial R_{n} \cap \Sigma=R_{n} \cap \partial \Sigma-\partial\left(R_{n} \cap \Sigma\right)=-\partial\left(R_{n} \cap \Sigma\right)
$$

since $\partial \Sigma=0$ in $B_{1}$. So

$$
\partial\left(R_{n} \cap \Sigma\right)(1)=\left(R_{n} \cap \Sigma\right)(d 1)=0,
$$

which implies $k\left(\partial R_{n}, \Sigma\right)=0$. 
Consider now $k\left(S_{n}, \Sigma\right)$ and recall that $\partial S_{n}=\partial T_{n}$. We have that $\operatorname{spt} \partial S_{n} \cap \Sigma=$ $\emptyset$ and $\operatorname{spt} S_{n} \cap \partial \Sigma=\emptyset$, so $0 \notin f\left(\operatorname{spt}\left(\partial\left(S_{n} \times \Sigma\right)\right)\right)$ and this index is well-defined and given by

$$
f_{*}\left(S_{n} \times \Sigma\right)=k\left(S_{n}, \Sigma\right) \llbracket B_{\varepsilon}(0) \rrbracket,
$$

where $f: \mathbb{R}^{5} \times \mathbb{R}^{5} \rightarrow \mathbb{R}^{5}$ is $f(x, y)=x-y$ and $\varepsilon$ is such that $B_{\varepsilon} \cap f\left(\operatorname{spt}\left(\partial\left(S_{n} \times\right.\right.\right.$ $\Sigma)))=\emptyset$; thanks to Lemma 3.2, $\varepsilon$ can be chosen independently of $n$. So, for a fixed $\varepsilon$, we have that

$$
f_{*}\left(S_{n} \times \Sigma\right)=k\left(S_{n}, \Sigma\right) \llbracket B_{\varepsilon}(0) \rrbracket
$$

holds for all $n$ large enough. By assumption we know that $M\left(S_{n}\right) \rightarrow 0$, therefore $M\left(S_{n} \times \Sigma\right) \rightarrow 0$ and $M\left(f_{*}\left(S_{n} \times \Sigma\right)\right) \rightarrow 0$ since $f$ is Lipschitz; but then, for $\varepsilon$ fixed and $k \in \mathbb{N}$, the only possibility for the r.h.s. of (3.3) to go to zero in mass-norm is that eventually $k\left(S_{n}, \Sigma\right)=0$. So we can conclude that $k\left(T_{n}, \Sigma\right)=0$ for all large enough $n$.

Remark 3.4. If $Q$ is the multiplicity at 0 and $\Sigma=\Sigma_{0}$ such that $\Sigma_{0}$ is transversal to all $D_{i}$ that constitute the tangent cone $C$, then $k\left(C_{i}, \Sigma_{0}\right)=Q$ for $i$ greater than some $i_{0}$. Once we have this, $k\left(C_{i}, \Sigma\right)=Q$ also holds for any 3-surface $\Sigma$ that can be joined to $\Sigma_{0}$ via a homotopy during which we do not cross $\partial C_{i}$, in particular for small translations $\tau_{a} \Sigma_{0}$.

\section{Uniqueness of the tangent cone - easy case of non-accumulation - Lipschitz estimate}

The uniqueness of the tangent cone at an arbitrary point of the Special Legendrian follows from the more general result proved in [16] for general semi-calibrated integral 2-cycles. In this section, using the tools developed in the previous sections, we will give a self-contained proof of this uniqueness in our situation. The section then continues with proofs in the same flavour of the two other results quoted in the title of the section.

The following lemma (see left picture in Figure 4.1) is stated separately since it will be repeatedly recalled in the several proofs of this section. $C$ is our Special Legendrian current.

Lemma 4.1. Let $p \in S^{5}$ and consider a polar foliation born at $p$, i.e. a family $\left(\Sigma_{p}^{X}\right)$ of 3-surfaces, for $X$ varying in an open ball $U$ of $\mathbb{C P} \mathbb{P}^{1} \cong \mathbb{P} H_{p}^{4}$. For $0<r<$ $R$, consider the open set

$$
W=\left(\cup_{X \in U} \Sigma_{p}^{X}\right) \cap\left(B_{R}(p)-\overline{B_{r}(p)}\right) .
$$

Assume that $C\left\llcorner W \neq 0\right.$ and that $\operatorname{spt}\left(\partial(C\llcorner W)) \subset \cup_{X \in \partial U} \Sigma_{p}^{X}\right.$. Then $k\left(C\left\llcorner W, \Sigma_{p}^{X}\right) \geq\right.$ 1 for any $X \in U$. 
Proof of Lemma 4.1. The carrier of $C\llcorner W$ is just $\mathcal{C} \cap W$, where $\mathcal{C}$ is the carrier of the Special Legendrian current.

Define $s: W \rightarrow U$ to be the smooth function taking the value $Y \in U$ at points of $\Sigma_{p}^{Y} \cap W$. From slicing and intersection theory we have the following facts.

- from [8, page 156], we know that the slice $\langle C\llcorner W, s=Y\rangle$ is well-defined for $\mathcal{H}^{2}$ almost all $Y \in U$ as a sum of Dirac deltas with integer weights, supported on the finite set of points $\mathcal{C} \cap W \cap s^{-1}\{Y\}=\mathcal{C} \cap W \cap \Sigma_{p}^{Y}$. The weight of each Dirac delta is just the multipliciticy of the Special Legendrian at that point, with a sign induced by the sign of the intersection of the oriented tangent to $C$ and the tangent to $\Sigma_{p}^{Y}$. The sign is always positive in our case, due to the positive intersection property of the foliation;

- recalling that $\left\langle C\llcorner W, s=Y\rangle=\left(C\llcorner W) \cap \Sigma_{p}^{Y}\right.\right.$, we have that, when the slice $\left\langle C\llcorner W, s=Y\rangle\right.$ exists, the Kronecker index $k\left(C\left\llcorner W, \Sigma_{p}^{Y}\right)\right.$ is just $\langle C\llcorner W, s=$ $Y\rangle(1)$, the sum of the weights of the Dirac deltas that appear in the slice. By the positiveness of intersections we then see that, as long as $\left(C\llcorner W) \cap \Sigma_{p}^{Y}\right.$ exists and $\mathcal{C} \cap W \cap \Sigma_{p}^{Y} \neq \emptyset$, the index $k\left(C\left\llcorner W, \Sigma_{p}^{Y}\right)\right.$ is strictly positive.

Observe further that, as soon as we have a particular $Y \in U$ for which $k\left(C\left\llcorner W, \Sigma_{p}^{Y}\right) \geq\right.$ 1 , we can say the same for any other $X \in U$, thanks to the hypotesis on the boundary of $C$ : indeed the 3-surfaces $\Sigma_{p}^{Y} \cap W$ and $\Sigma_{p}^{X} \cap W$, for any $X, Y \in U$, can be connected by homotopy without crossing $\operatorname{spt}(\partial(C\llcorner W))$, therefore the intersection index stays constant.

In view of the observations made, it is enough to have the strict positiveness of $k\left(C\left\llcorner W, \Sigma_{p}^{Y}\right)\right.$ for just a single $Y \in U$ in order to conclude the proof of the lemma. Therefore we ask: is it possible that, for almost all $X \in U$ the intersection $\mathcal{C} \cap W \cap \Sigma_{p}^{X}$ is empty? Let us analyse what should happen in this case.

If for $\mathcal{H}^{2}$-almost all $X \in U$ the forementioned interection is empty, we would find by the coarea formula (see [8, Theorem 3, pages 102-103]) that

$$
\int_{\mathcal{C} \cap W} J_{s}^{\mathcal{C}} d \mathcal{H}^{2}=\int_{U}\left\{\int_{s^{-1}\{X\}} d \mathcal{H}^{0}\right\} d \mathcal{H}^{2}(X)=0,
$$

where $J_{s}^{\mathcal{C}}$ is the Jacobian of $s$ relative to the approximate tangent of $\mathcal{C}$. The formula would imply that $\mathcal{H}^{2}$ almost everywhere on $\mathcal{C} \cap W$ it must hold $J_{s}^{\mathcal{C}}=0$, so that each approximate tangent to $\mathcal{C}$ must have at least a direction in common with the tangent to $\Sigma_{p}^{X}$ : but thanks to the pseudo-holomorphic behaviour from proposition 2.1 and the way the 3-surfaces are constructed, this would then force, at almost all points of $\mathcal{C} \cap W, \mathcal{C}$ to be tangent to the 3 -surfaces $\Sigma_{p}^{X}$.

It could be proved directly that this is impossible, since it would force $C$ to be made of a sum of Special Legendrian spheres (some of those building up the 3-surfaces $\Sigma^{X}$ ), and $C$ would therefore have boundary on $\partial B_{r}$ and $\partial B_{R}$, contradiction. 

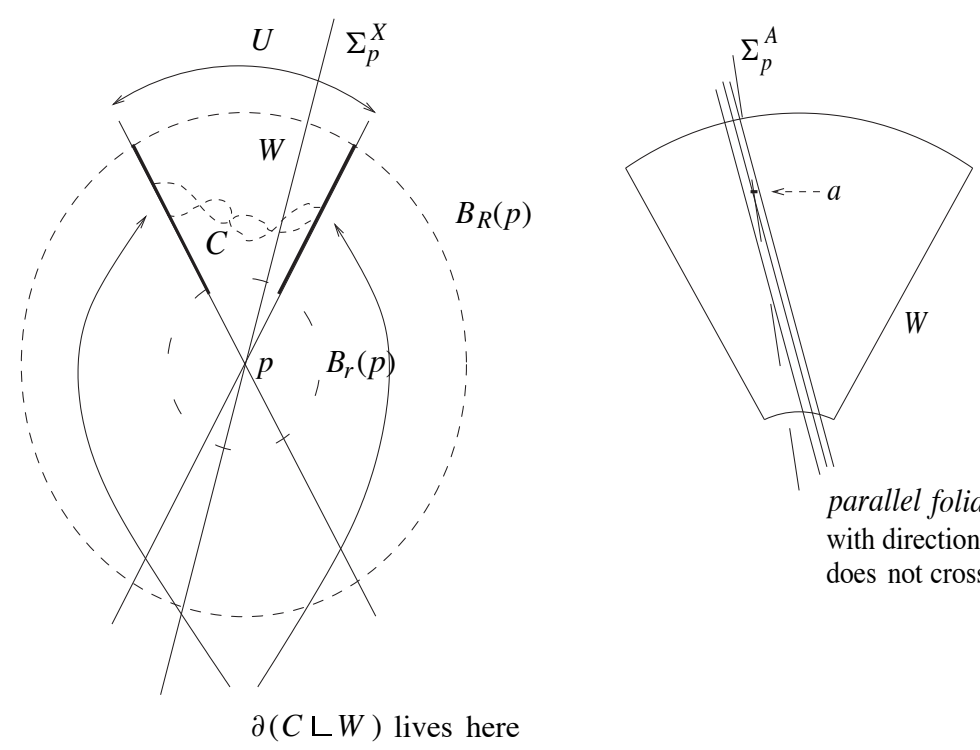

parallel foliation with direction $Z$ at $a$ does not cross $\partial(C\llcorner W)$

Figure 4.1. On the left: schematical view of the statement. $C$ is dashed. Due to the condition on the boundary of $C\left\llcorner W\right.$, for any $\Sigma_{p}^{X}$ with $X \in U$ we must find a strictly positive intersection index. On the right: the choice of the parallel foliation near $a$.

We prefer however to avoid the technicalities of that proof, and show just the content of the lemma: this can be achieved as follows.

We have seen that, if for $\mathcal{H}^{2}$-almost all $X \in U$ it is true that $\mathcal{C} \cap W \cap \Sigma_{p}^{X}=\emptyset$, then for $\mathcal{H}^{2}$ a.e. $q \in \mathcal{C} \cap W$ we must have $T_{q} \mathcal{C} \subset T_{q} \Sigma_{p}^{X}$, for the unique $X$ such that $q \in \Sigma_{p}^{X}$.

Take a point $a \in \mathcal{C} \cap W$ having density 1 with respect to $\mathcal{H}^{2}$. It exists since $\mathcal{C} \cap W$ is non-empty. Denote by $\Sigma_{p}^{A}$ the 3 -surface born at $p$ passing through $a$.

Now take a 3 -surface $\Sigma_{a}^{Z}$ born at $a$, where $Z$ is a direction in $\mathbb{C P}^{1} \cong \mathbb{P} H_{a}^{4}$ taken such that $\Sigma_{a}^{Z}$ is transversal to $\Sigma_{p}^{A}$ and $\Sigma_{a}^{Z} \cap \partial W$ is disjoint from $\cup_{X \in \partial U} \Sigma_{p}^{X}$, in particular disjoint from $\operatorname{spt}\left(\partial(C\llcorner W))\right.$. To ensure that, it is enough to take $\Sigma_{a}^{Z}$ close enough to $\Sigma_{p}^{A}$.

Take a parallel foliation of 3-surfaces $\Sigma_{w}^{Z}$ parallel to $\Sigma_{a}^{Z}$. Choose this parallel foliation such that all the $\Sigma_{w}^{Z}$ do not intersect $\operatorname{spt}(\partial(C\llcorner W)$ ) (Figure 4.1, picture on the right), which is ensured if these parallel 3-surfaces stay close enough to $\Sigma_{a}^{Z}$. Any small enough neighbourhood $V_{a}$ of $a$ is foliated by these parallel 3-surfaces $\Sigma_{w}^{Z}$

Claim. It is not possible that for $\mathcal{H}^{2}$-almost every 3-surface of the parallel foliation it happens $\mathcal{C} \cap V_{a} \cap \Sigma_{w}^{Z}=\emptyset$. 
Indeed, if this were the case, we would find, by means of the coarea formula as above, that $\mathcal{H}^{2}$-almost all of $\mathcal{C} \cap V_{a}$ is tangent to the 3-surfaces $\Sigma_{w}^{Z}$ (remark that, no matter how small $V_{a}$ is, $\mathcal{H}^{2}\left(\mathcal{C} \cap V_{a}\right)>0$ since $a$ has density 1$)$.

But $\mathcal{C} \cap V_{a}$ cannot simultaneusly be tangent to the $\Sigma_{w}^{Z}$ 's and to the $\Sigma_{p}^{X}$ 's. Indeed, $\Sigma_{a}^{Z}$ was chosen transversal to $\Sigma_{p}^{A}$, so if $V_{a}$ is small enough, inside $V_{a}$ we have that, by stability of the transversality, all the $\Sigma_{p}^{X}$ are transversal to all the $\Sigma_{w}^{Z}$. This proves the claim.

So we can find a $\mathcal{H}^{2}$-positive set of $\Sigma_{w}^{Z}$ such that $\mathcal{C} \cap V_{a} \cap \Sigma_{w}^{Z} \neq \emptyset$. Now, looking at the situation in the whole of $W$, for $\mathcal{H}^{2}$-almost all the $\Sigma_{w}^{Z}$ 's, the intersection $C \cap \Sigma_{w}^{Z}$ is well-defined and the Kronecker index $k\left(C\left\llcorner W, \Sigma_{w}^{Z}\right)=((C\llcorner W) \cap\right.$ $\left.\Sigma_{w}^{Z}\right)(1)$ must be $\geq 1$ due to the strictly positive contribution in $V_{a}$.

But $\Sigma_{w}^{Z}$ and $\Sigma_{p}^{A}$ can be joined by homotopy without crossing the boundary of $C\left\llcorner W\right.$, therefore the index stays constant during the homotopy and $k\left(C\left\llcorner W, \Sigma_{p}^{A}\right) \geq\right.$ 1. Again by homotopy, we find that for any $Z \in U$ the index $k\left(C\left\llcorner W, \Sigma_{p}^{X}\right)\right.$ is a striclty positive integer, concluding the proof.

Uniqueness of the tangent cone. We start with the following:

Lemma 4.2. Take any point $x_{0}$ of a Special Legendrian cycle $C$ and be $Q$ its multiplicity. Then there exists a unique choice of $n$ distinct Special Legendrian disks $D_{1}, \ldots D_{n}$ going through $x_{0}$ such that any tangent cone at $x_{0}$ must be of the form $T_{x_{0}} C=\oplus_{k=1}^{n} N_{k} D_{k}$, for some $N_{k} \in \mathbb{N} \backslash\{0\}$ satisfying $\sum_{k=1}^{n} N_{k}=Q$.

Remark 4.3. This result "almost" gives the uniqueness of the tangent cone. What still is missing, is the fact that the multiplicities $N_{k}$ are also uniquely determined. This will be achieved in Theorem 4.5.

Proof of Lemma 4.2. We work in a normal chart centered at the origin, so that 0 has multiplicity $Q$. With a little abuse of notation, we will write $C\left\llcorner B_{r}(0)\right.$ (for small enough $r$ ) meaning the current, restricted to the geodesic ball of radius $r$, seen in the chart.

Argue by contradiction: take two tangent cones $C_{\infty}^{(1)}=\oplus_{k=1}^{n_{1}} N_{k}^{(1)} D_{k}^{(1)}$ and $C_{\infty}^{(2)}=\oplus_{k=1}^{n_{2}} N_{k}^{(2)} D_{k}^{(2)}$ having distinct supports, and two blown-up sequences $\left\{C_{x_{0}, r_{i}}\right\}$ and $\left\{C_{x_{0}, \rho_{i}}\right\}$ converging to each of them. In this proof we denote $C_{x_{0}, r}\left\llcorner B_{1}(0)\right.$ simply by $C_{r}$, so

$$
C_{r_{i}} \rightarrow C_{\infty}^{(1)}, \quad C_{\rho_{i}} \rightarrow C_{\infty}^{(2)}
$$

As the proof goes on, the reader might refer to Figure 4.2 for a schematic visualization of the objects involved.

Take a positive $\delta$ much smaller than the angular distance

$$
C_{\infty}^{(1), C_{\infty}^{(2)}}:=\min _{D_{i} \neq D_{j}} \widehat{D_{i}^{(1)}, D_{j}^{(2)}} \gg \delta>0
$$


(the distance is given by the Fubini-Study metric in $\mathbb{C P}^{1} \cong \mathbb{P} H_{0}^{4}$ and is strictly positive by the contradiction assumption). Moreover assume, without loss of generality, that the disk of $C_{\infty}^{(1)}$ on which the minimum is achieved is $D_{0}$, the disk represented by $[1,0] \in \mathbb{C P}^{1}$. In particular we are also assuming that $D_{0}$ is not in the support of $C_{\infty}^{(2)}$. By abuse of notation we will write $D_{0} \in C_{\infty}^{(1)}$ to express the fact that $D_{0}$ is one of the disks that build up the cone $C_{\infty}^{(1)}$. Analogously we have $D_{0} \notin C_{\infty}^{(2)}$. Choose $\rho_{i_{0}}$ such that

(i) for $j \geq i_{0}, \partial\left(C\left\llcorner B_{\rho_{j}}\right)\right.$ is contained in $E_{2}^{\delta}$, the $\delta$-conic-neighbourhood of $C_{\infty}^{(2)}$ (possible by Lemma 3.2);

(ii) $k\left(C_{\rho_{j}}, \Sigma_{0}^{[1,0]}\right)=Q$ for any $j \geq i_{0}$. Remark that $\Sigma_{0}^{[1,0]}$ is transversal to $C_{\infty}^{(2)}$. By homotopy, it also holds that $k\left(C_{\rho_{j}}, \Sigma_{0}^{X}\right)=Q$ for any $j \geq i_{0}$ and any $\Sigma_{0}^{X}$ with $X \in \mathbb{C P}^{1}$ in a $\delta$-neighbourhood of $[1,0]$. Indeed, the homotopy keeps the condition of non-crossing boundaries expressed in (3.2).

Choose now $r_{i_{1}}<\rho_{i_{0}}$ such that

(iii) denoting by $E_{0}^{\delta}$ the $\delta$-conic-neighbourhood of $D_{0}$ and setting

$$
W_{1}=\left(B_{r_{i_{1}}} \backslash B_{\frac{r_{i_{1}}}{2}}\right) \cap E_{0}^{\delta},
$$

we have

$$
C\left\llcorner W_{1} \neq 0\right.
$$

this is true for $i$ large enough since $C_{r_{i}} \rightarrow C_{\infty}^{(1)} \ni D_{0}$.

Take now $\rho_{i_{1}} \ll \frac{r_{i_{1}}}{2}$. Define

$$
W_{2}:=\left(B_{\rho_{i_{0}}} \backslash B_{\rho_{i_{1}}}\right) \cap E_{0}^{\delta} \supset W_{1} .
$$

$W_{2}$ is foliated by $\Sigma_{0}^{X}$ as $X$ varies in a $\delta$-neighbourhood of [1,0].

From (i), $\partial\left(C\left\llcorner\left(B_{\rho_{i_{0}}} \backslash B_{\rho_{i_{1}}}\right)\right)\right.$ is zero on $\overline{W_{2}} \cap \partial B_{R}$ and on $\overline{W_{2}} \cap \partial B_{r}$.

From (iii) we know that $C\left\llcorner W_{2} \neq 0\right.$.

So we can use Lemma 4.1 in the open set $W_{2}$. Then, for almost all $X$ in the $\delta$-neighbourhood of $[0,1]$, we have

$$
\begin{aligned}
k\left(C\left\llcorner B_{\rho_{i_{0}}}, \Sigma_{0}^{X}\right)\right. & =k\left(C\left\llcorner B_{\rho_{i_{1}}}, \Sigma_{0}^{X}\right)+k\left(C\left\llcorner\left(B_{\rho_{i_{0}}} \backslash B_{\rho_{i_{1}}}\right), \Sigma_{0}^{X}\right)\right.\right. \\
& =k\left(C_{\rho_{i_{1}}}, \Sigma_{0}^{X}\right)+k\left(C\left\llcorner W_{2}, \Sigma_{0}^{X}\right)+k\left(C\left\llcorner\left(\left(B_{\rho_{i_{0}}} \backslash B_{\rho_{i_{1}}}\right) \backslash W_{2}\right), \Sigma_{0}^{X}\right)\right.\right. \\
& =Q+k\left(C\left\llcorner W_{2}, \Sigma_{0}^{X}\right)+k\left(C\left\llcorner\left(\left(B_{\rho_{i_{0}}} \backslash B_{\rho_{i_{1}}}\right) \backslash W_{2}\right), \Sigma_{0}^{X}\right)\right.\right. \\
& \geq Q+1 ;
\end{aligned}
$$

the last inequality follows from the positivity $(\geq 0)$ of intersection in $\left(B_{\rho_{i_{0}}} \backslash B_{\rho_{i_{1}}}\right) \backslash$ $W_{2}$ and the strict positiveness $(\geq 1)$ guaranteed in $W_{2}$. This contradicts (ii). 


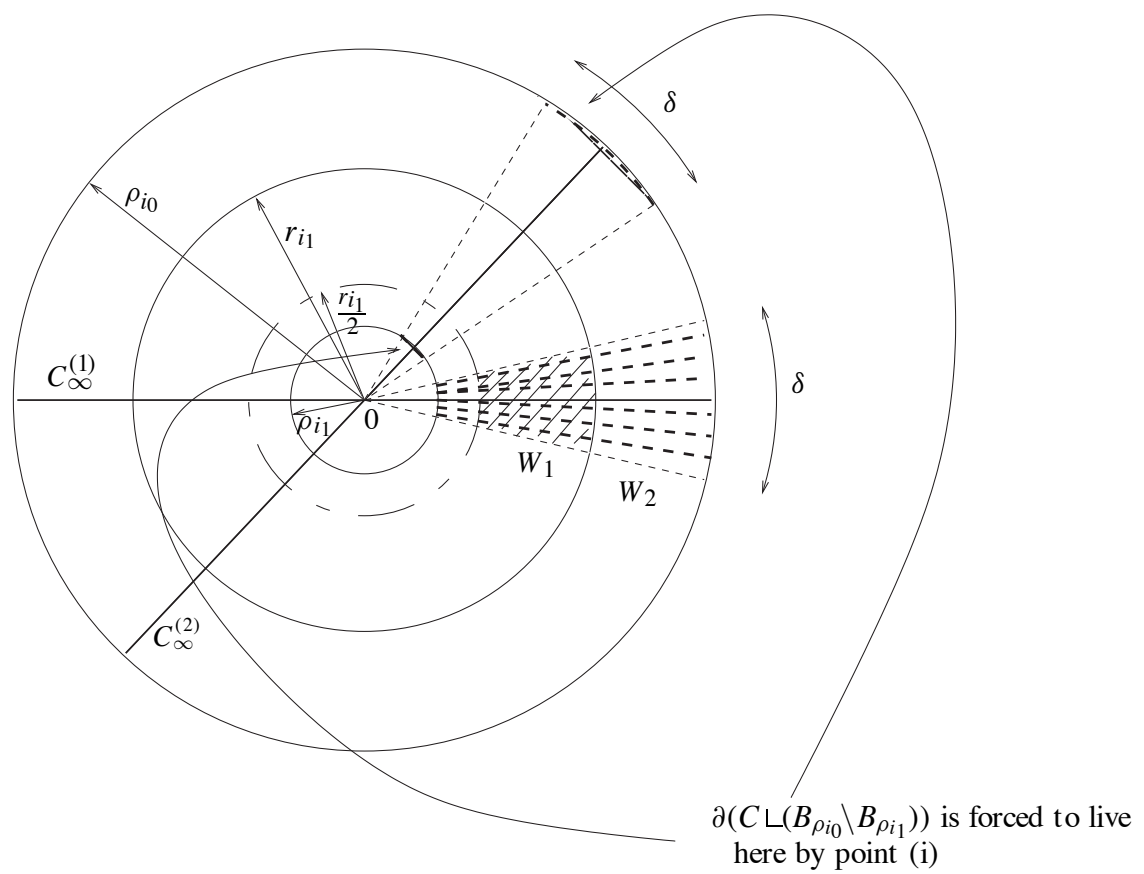

Figure 4.2. In the picture we have taken $C_{\infty}^{(1)}$ to be $Q$ times $D_{0} . C_{\infty}^{(2)}$ is a different disk counted $Q$ times. The horizontal and vertical directions should be respectively thought of as $[1,0]$ and $[0,1]$. The fifth direction should be imagined as entering the picture. The dotted region corresponds to $W_{2}$; its subset $W_{1}$ is shaded.

Now that this "almost uniqueness" of the tangent cone is established, we can improve Lemma 3.2 as follows:

Lemma 4.4. Let $\left\{D_{k}\right\}_{k=1}^{n}$ be the uniquely determined disks on which any tangent cone to $C$ at $x_{0}$ must be supported. Let us therefore write $T=\cup_{k} D_{k}$ for this welldetermined support. Denote by $E_{\varepsilon}$ the cone $\left\{x \in B_{1}\right.$, $\left.\operatorname{dist}(x, T)<\varepsilon|x|\right\}$. Then for any $\varepsilon>0$ there is $\rho_{\varepsilon}$ small enough such that for any $\rho \leq \rho_{\varepsilon}$

$$
\operatorname{spt}\left(C_{x_{0}, \rho}\left\llcorner B_{1}(0)\right) \backslash\{0\} \subset E_{\varepsilon} .\right.
$$

Proof of Lemma 4.4. The proof is similar to the one of Lemma 3.2. Assume the existence of $\varepsilon_{0}>0$ and $\rho_{n} \rightarrow 0$ contradicting the claim and argue as in the proof of Lemma 3.2. The only modification in the proof consists in using the "almost uniqueness" of the tangent cone at 0 (Lemma 4.2) instead of the condition $R \leq$ $\frac{\rho_{n}\left|x_{n}\right|}{\rho_{n}} \leq 1$. If $C_{x_{0}, \rho_{n}}$ converges to the cone $C_{\infty}=\oplus_{k=1}^{n} N_{k} D_{k}$, then $C_{x_{0}, \rho_{n}\left|x_{n}\right|}$ must tend to a limiting cone $\tilde{C}_{\infty}=\oplus_{k=1}^{n} \tilde{N}_{k} D_{k}$. So the computation in (3.1) can be performed with $\tilde{C}_{\infty}$ instead of $C_{\infty}$, still leading to a contradiction since the supports of $\tilde{C}_{\infty}$ and $C_{\infty}$ are the same. 
Now we can complete the proof of the uniqueness of the tangent cone:

Theorem 4.5. The tangent cone at any point $x_{0}$ of a Special Legendrian cycle $C$ is unique.

Proof of Theorem 4.5. With the result and the notations of Lemma 4.2 in mind, we only have to exclude that the multiplicities $N_{k}$ may depend on the chosen sequence that we blow-up.

Choose $\varepsilon$ small enough to ensure that different $\varepsilon$-neighbourhoods

$$
E_{\varepsilon}^{i}=\left\{x \in B_{1}, \operatorname{dist}\left(x, D_{i}\right)<\varepsilon|x|\right\}, \quad E_{\varepsilon}^{j}=\left\{x \in B_{1}, \operatorname{dist}\left(x, D_{j}\right)<\varepsilon|x|\right\}
$$

of different disks $D_{i}$ and $D_{j}$ do not overlap, i.e. $E_{\varepsilon}^{i} \cap E_{\varepsilon}^{j}=\emptyset$.

Rotate $B_{1}$ in order to have that the family $\Sigma_{p}:=\Sigma_{p}^{[0,1]}$ is transversal to all the disks $D_{k}$. Then, for $p$ in a neighbourhood $B_{\delta}$ of 0 and for all small enough $r$, the index $k\left(C_{r}, \Sigma_{p}\right)$ is well-defined since Lemma 4.4 ensures the condition (3.2) of non-crossing-boundaries.

The key observation is that the rescaled $C_{r}$ form a continuous (with respect to $r$ ) family of currents (with respect to the flat-topology) and they are always constrained in the $E_{\varepsilon}$-neighbourhood given by Lemma 4.4. Fix $i$ : the fact that the $E_{\varepsilon}^{k}$ are well separated implies that, for any $p \in B_{\delta}$,

$$
\partial\left(C_{r}\left\llcorner E_{\varepsilon}^{i}\right) \cap \Sigma_{p}=\emptyset, \quad\left(C_{r}\left\llcorner E_{\varepsilon}^{i}\right) \cap \partial \Sigma_{p}=\emptyset .\right.\right.
$$

Moreover, due to the mentioned continuity, as $r \rightarrow 0$ the currents $C_{r}\left\llcorner E_{\varepsilon}^{i}\right.$ are all homotopic to each other, and these homotopies keep the condition (3.2) between $C_{r}\left\llcorner E_{\varepsilon}^{i}\right.$ and $\Sigma_{p}\left\llcorner E_{\varepsilon}^{i}\right.$.

Therefore $k\left(C_{r}\left\llcorner E_{\varepsilon}^{i}, \Sigma_{p}\right)\right.$ must stay constant as $r \rightarrow 0$, so there is a welldetermined $N_{i} \in \mathbb{N}$ such that $k\left(C_{r}\left\llcorner E_{\varepsilon}^{i}, \Sigma_{p}\right)=N_{i}\right.$. Then any limiting cone $C_{\infty}$ must satisfy $k\left(C_{\infty}\left\llcorner E_{\varepsilon}^{i}, \Sigma_{p}\right)=N_{i}\right.$, with the same proof as in Lemma 3.3. This means that $C_{\infty}\left\llcorner E_{\varepsilon}^{i}=N_{i} D_{i}\right.$, so all the multiplicities $N_{k}$ are uniquely determined.

Easy case of non-accumulation. The following result solves the "easy case" of non-accumulation of singularities of multiplicity $Q$ to a singularity $p$ of the same multiplicity: this "easy case" arises when the tangent cone at $p$ is not made of $Q$ times the same disk. We will see how to handle the "difficult case" (tangent cone made of $Q$ times the same plane) in Sections 5 and 6 .

Define the set $\operatorname{Sing}^{Q}$ of singularities of multiplicity (or order) $Q$ of the Special Legendrian cycle $C$ :

$$
\operatorname{Sing}^{Q}:=\{p \in C: p \text { is a singular point, } \theta(p)=Q\} .
$$

In the same fashion we will use the notation

$$
\text { Sing } \leq Q:=\{p \in C: p \text { is a singular point }, \theta(p) \leq Q\} .
$$


Theorem 4.6. For a Special Legendrian cycle $C$, assume $x_{0} \in \operatorname{Sing} Q, T_{x_{0}} C \neq$ $Q \llbracket D \rrbracket$, i.e. $T_{x_{0}} C=\oplus_{k=1}^{m} N_{k} D_{k}$, where $D_{k}$ are distinct Special Legendrian disks and $m \geq 2$. Then $\exists r>0$ such that

$$
\text { Sing } Q \cap B_{r}\left(x_{0}\right)=\left\{x_{0}\right\} .
$$

Proof of Theorem 4.6. The proof uses techniques similar to those from Theorem 4.5. Take a normal chart with $x_{0}=0$ : by contradiction, assume $\exists x_{n} \rightarrow 0$, with $x_{n} \in \operatorname{Sing} Q$. Rename the $D_{i}$ 's so that $D_{1}$ and $D_{2}$ realize the minimum $\gamma$ of the angular distances $\widehat{D_{i}, D_{j}} \cdot \gamma>0$ since $T_{0} C \neq Q \llbracket D \rrbracket$ and $\gamma \leq \frac{\pi}{2}$ since this is the maximum for the Fubini-Study metric.

Define $\rho_{n}=2\left|x_{n}\right|$ and blow up about 0 using $\rho_{n}$ as rescaling factors. Up to a possible exchange of the roles of $D_{1}$ and $D_{2}$ and up to a subsequence, we can assume $\frac{x_{n}}{2\left|x_{n}\right|} \rightarrow p \in D_{2} \cap \partial B_{1 / 2}$. Rotate $B_{1}$ to ensure that $D_{1}$ and $D_{2}$ are contained in the $\frac{3 \pi}{4}$-cone around $D_{0} \cong[1,0]$ and that $\Sigma_{p}^{[0,1]}$ is transversal to the disks $\left\{D_{j}\right\}_{j=1}^{m}$. The situation is schematically described in Figure 4.3 (read the caption for some heuristics of the proof).

Take $\alpha \ll \gamma$; for all $n$ large enough, thanks to Lemma 4.4 we can ensure that

$$
\operatorname{spt}\left(C_{x_{0}, \rho_{n}}\left\llcorner B_{1}\right) \subset \cup_{i=1}^{m} E_{i}^{\alpha} \cup\{0\},\right.
$$

where $E_{i}^{\alpha}$ denotes the cone of width $\alpha$ around $D_{i}$. Thanks to the position of $D_{1}$ and $D_{2}$, we can find a small enough ball $U \subset \mathbb{C} \mathbb{P}^{1}$ centered at $[0,1]$ such that for any $X \in U$ we have that $\Sigma_{p}^{X}$ is transversal to the disks $\left\{D_{j}\right\}_{j=1}^{m}$ and that $\Sigma_{p}^{X} \cap$ $\operatorname{spt}\left(\partial\left(C_{x_{0}, \rho_{n}}\left\llcorner B_{1}\right)\right)=\emptyset\right.$.

In this situation, thanks to Lemma 3.3, we know that for $X \in U$ and for all large enough $n$

$$
k\left(C_{x_{0}, \rho_{n}}\left\llcorner B_{1}, \Sigma_{p}^{X}\right)=k\left(T_{x_{0}} C, \Sigma_{p}^{X}\right)=\sum_{j=1}^{n} N_{j} k\left(D_{j}, \Sigma_{p}^{X}\right) \leq Q\right.
$$

Let $V$ be a ball strictly smaller than $U$ with the same center and define

$$
W:=E_{1}^{\alpha} \cap\left(\cup_{X \in V} \Sigma_{p}^{X}\right) .
$$

At each $y_{n}$ choose an open ball $V_{n} \subset \mathbb{C P}^{1} \cong \mathbb{P} H_{y_{n}}^{4}$ so that, for $n \geq n_{0}$ large enough,

(i) $\forall X \in V_{n}$ we have that $\Sigma_{y_{n}}^{X}$ is transversal to the disks $\left\{D_{j}\right\}_{j=1}^{m}$ and that $\Sigma_{y_{n}}^{X} \cap$ $\operatorname{spt}\left(\partial\left(C_{x_{0}, \rho_{n}}\left\llcorner B_{1}\right)\right)=\emptyset\right.$;

(ii) setting $W_{n}:=E_{1}^{\alpha} \cap\left(\cup_{X \in V_{n}} \Sigma_{y_{n}}^{X}\right)$, it holds $W \subset \cap_{n \geq n_{0}} W_{n}$.

Properties (i) and (ii) can of course be achieved for $y_{n}$ close enough to $p$ and $V_{n}$ perturbations of $V$. 


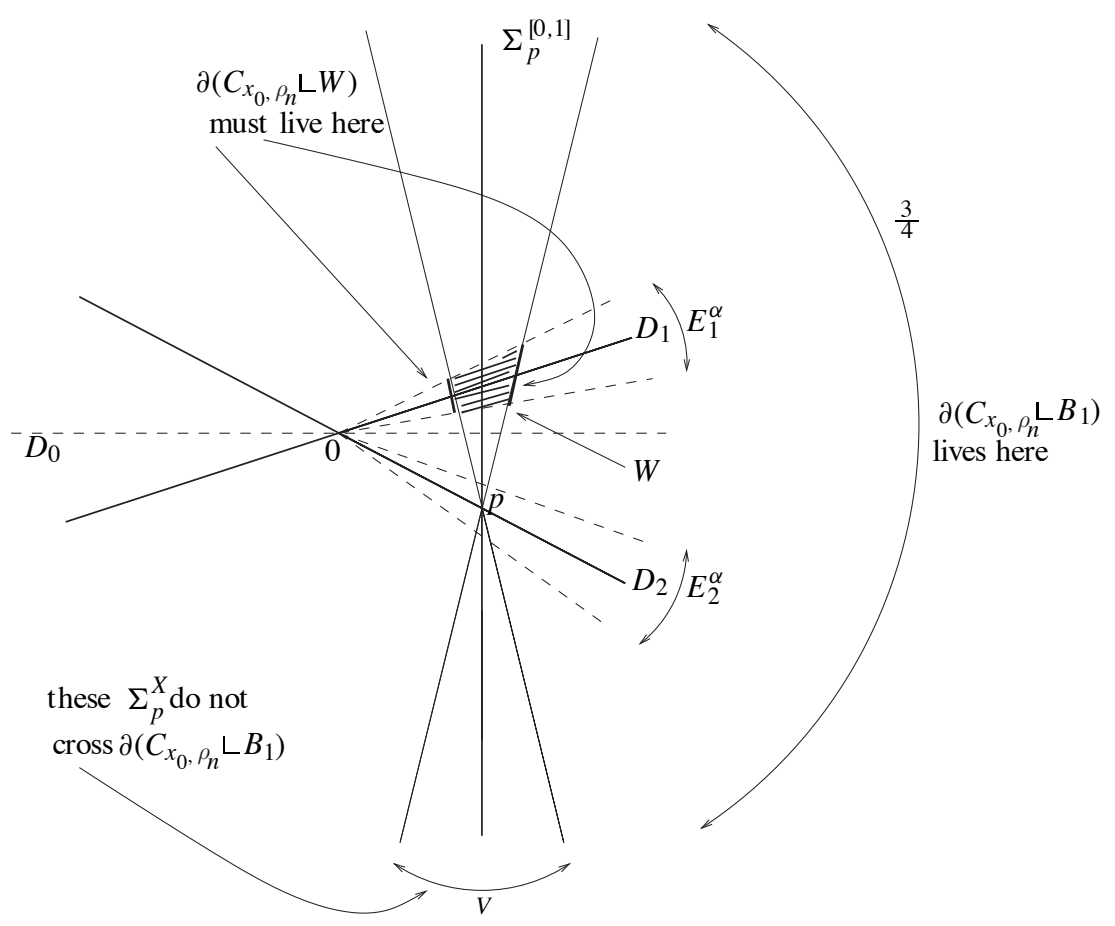

Figure 4.3. Situation inside the ball $B_{1}$. To avoid confusion in the picture, we imagine that $y_{n}$ coincides with $p$. The dotted region corresponds to $W$.

Heuristic idea of the proof: any $\Sigma_{p}^{X}, X \in V$, should intersect $C_{x_{0}, \rho_{n}}$ with multiplicity $Q$ near $p$, since there we have a point of multiplicity $Q$. But there is mass of $C_{x_{0}, \rho_{n}}$ in $W$ and this portion must also give a strictly positive contribution to the intersection index of $C_{x_{0}, \rho_{n}}$ and $\Sigma_{p}^{X}$. But now there is too much intersection.

Thanks to the convergence $C_{x_{0}, \rho_{n}} \rightarrow T_{x_{0}} C \ni D_{1}$, we can ensure that for all $n$ large enough

$$
C_{x_{0}, \rho_{n}}\llcorner W \neq 0,
$$

which trivially implies $C_{x_{0}, \rho_{n}}\left\llcorner W_{n} \neq 0\right.$.

$W_{n}$ is foliated by $\cup_{X \in V_{n}} \Sigma_{y_{n}}^{X}$ and $\partial\left(C_{x_{0}, \rho_{n}}\left\llcorner W_{n}\right) \subset \cup_{X \in \partial V_{n}} \Sigma_{y_{n}}^{X}\right.$ by (4.1). Then by Lemma 4.1 we have that, for all $Y \in V_{n}$,

$$
k\left(C_{x_{0}, \rho_{n}}\left\llcorner W_{n}, \Sigma_{y_{n}}^{Y}\right) \geq 1 .\right.
$$

On the other hand, recalling Remark 3.4, for $\varepsilon$ small enough it must hold $k\left(C_{x_{0}, \rho_{n}}\left\llcorner B_{\varepsilon}\left(y_{n}\right), \Sigma_{y_{n}}^{Y}\right)=Q\right.$ for all but finitely many $Y$ 's (we only have to exclude the $Y$ 's that build up $\left.T_{y_{n}} C\right)$. Since $W_{n} \cap B_{\varepsilon}\left(y_{0}\right)=\emptyset$, we get

$$
k\left(C_{x_{0}, \rho_{n}}\left\llcorner B_{1}, \Sigma_{y_{n}}^{Y}\right) \geq Q+1\right.
$$


But then

$$
k\left(C_{x_{0}, \rho_{n}}\left\llcorner B_{1}, \Sigma_{p}^{Y}\right) \geq Q+1\right.
$$

by homotopy (by (i) and (ii) we do not cross the boundary of $C_{x_{0}, \rho_{n}}\left\llcorner B_{1}\right.$ during the homotopy). This contradicts (4.2).

Relative Lipschitz-type estimate. The following theorem still uses the same ideas and will be of central importance for treating the more delicate case of a singular point $p$ having a tangent cone that is $Q$ times the same plane. We can without loss of generality assume that the plane involved is $D_{0} \cong[1,0]$. The result shows the "continuous behaviour" of tangent cones at points of multiplicity $Q$ as they approach $p$ (see Figure 5.1 in the next section).

Theorem 4.7. Let $x_{0}$ be a singular point of order $Q$ of a Special Legendrian cycle, $x_{0} \in \operatorname{Sing} Q$, with $T_{x_{0}} C=Q \llbracket D_{0} \rrbracket$. Then $\forall\left\{y_{n}\right\} \rightarrow x_{0}$ sequence of points having multiplicity $Q$, the following holds:

$$
T_{y_{n}} C \rightarrow Q \llbracket D_{0} \rrbracket
$$

Remark 4.8. The convergence in the statement can of course be understood in the Flat-sense for currents in the tangent bundle and what we are proving is:

$$
\forall \varepsilon \exists \delta \text { s.t. }\left|x-x_{0}\right|<\delta \text { and } \theta(x)=Q \Rightarrow \mathcal{F}\left(\left(T_{x} C-Q \llbracket D_{0} \rrbracket\right)\left\llcorner B_{1}\left(x_{0}\right)\right)<\varepsilon\right. \text {. }
$$

We give however a more concrete definition in terms of "angles" between the disks.

We are going to speak of "the angle between $D_{0}$ and $D_{p}$ " although these disks may lie in the horizontal hyperplanes at different points. More precisely: let $D_{0} \subset H_{x_{0}}^{4}$ and $D_{p} \subset H_{p}^{4}$ be holomorphic disks for the respective J-structures. Then we can define $\widehat{D_{p}, D_{0}}$ after identifying the two hyperplanes according to the coordinates induced by the first parallel foliation, see (2.2) in Section 2 (we can assume, without loss of generality $\left.x_{0}=(1,0,0)\right)$, and taking the distance in the Fubini-Study metric. The convergence in the theorem above amounts of course to the fact that the angles between $D_{0}$ and the disks of $T_{x_{n}} C$ go to 0 .

In the same fashion we will speak of $\widehat{\Sigma_{p}^{X}, D_{0}}$ for some 3-surface born at $p$, meaning the angle between $X$ and $D_{0}$ as just explained.

Proof of Theorem 4.7. Work in a normal chart centered at $x_{0}$. Assume, by contradiction, that there exists $\left\{y_{n}\right\} \rightarrow 0$ such that $T_{y_{n}} C \not \rightarrow Q \llbracket D_{0} \rrbracket$. Take as rescaling factors $\rho_{n}=2\left|y_{n}\right|$ and blow up about 0 . Denote $x_{n}=\frac{y_{n}}{2\left|y_{n}\right|}$ and keep denoting $\oplus_{i=1}^{Q} D_{n}^{i}$ the tangent disks at $x_{n}$. Now, up to a subsequence, for some $\alpha>0$, $\widehat{D_{n}^{i}, D_{0}} \geq \alpha>0$ and $x_{n} \in \partial B_{1 / 2}$ hold for all $n$. Choose $\varepsilon \ll \alpha$ such that $E_{0}^{\varepsilon} \cap \partial B_{1}$ is disjoint from any $\Sigma_{p}$ of the set $\left\{\Sigma_{p} \mid p \in E_{0}^{\varepsilon} \cap \partial B_{1 / 2}, \widehat{\Sigma_{p}, D_{0}} \geq \frac{\alpha}{2}\right\}$, see Figure 4.4. For a large enough $n$

(i) $C_{x_{0}, \rho_{n}}\left\llcorner B_{1} \subset E_{0}^{\varepsilon} \cup\{0\}\right.$ by Lemma 4.4, 


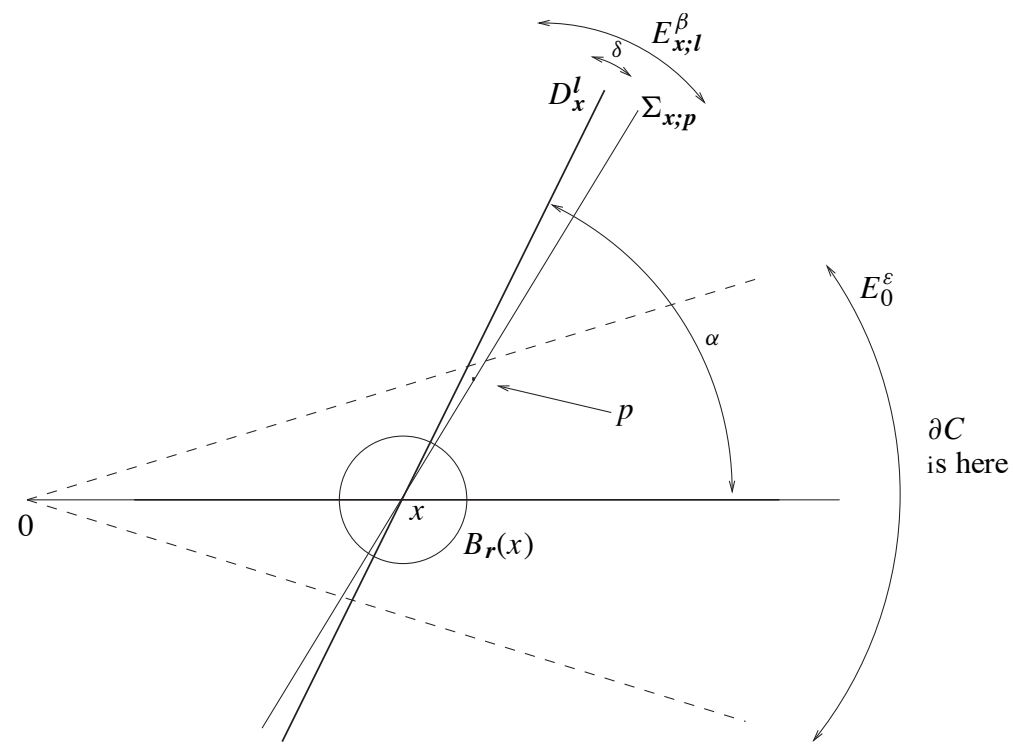

Figure 4.4. The objects involved: with $C$, as in the proof, we mean $C_{x_{0}, \rho_{n}}\left\llcorner B_{1}\right.$.

(ii) $k\left(C_{x_{0}, \rho_{n}}\left\llcorner B_{1}, \Sigma_{q}\right)=Q \quad \forall \Sigma_{q}\right.$ with $\widehat{\Sigma_{q}, D_{0}} \geq \frac{\alpha}{2}$ and $q \in D_{0} \cap B_{3 / 4}(0)$ by Lemma 3.3 and Remark 3.4.

Notational remark: the $n$ fulfilling (i) and (ii) is chosen once for all. Therefore we are going to drop, in the rest of this proof, the index $n$ from all the objects related to $x_{n}$, in particular we will denote by $x$ the point $x_{n}$ itself, by $T_{x} C=T_{x} C_{x_{0}, \rho_{n}}=$ $\oplus_{i=1}^{m} N_{i} D_{x}^{i}$ (the total multiplicity is $Q$ ) the tangent cone at $x_{n}$ and by $\Sigma_{x}^{i}$ the 3surface born at $x_{n}$ and containing $D_{x}^{i}$. Moreover, since the theorem is local, it is enough to look just at the dilated current $C_{x_{0}, \rho_{n}}\left\llcorner B_{1}(0)\right.$ : by an abuse of notation we will write, during this proof, $C$ instead of $C_{x_{0}, \rho_{n}}\left\llcorner B_{1}(0)\right.$.

From the contradiction assumption, at least for one index $l, \widehat{D_{x}^{l}, D_{0}}$ is greater than a positive number very close to $\alpha$.

Observe now the following: Take $\beta \ll \min _{i \neq j}\left\{\alpha, \widehat{D_{x}^{i}, D_{x}^{j}}\right\}$. Consider the cone $E_{x, l}^{\beta}$ around $\Sigma_{x}^{l}$. It is not possible that $\operatorname{spt}\left(C\left\llcorner E_{x, l}^{\beta}\right) \subset \Sigma_{x}^{l}\right.$ : indeed, this would imply that the current $C\left\llcorner E_{x, l}^{\beta}\right.$ must escape the barrier $E_{0}^{\varepsilon}$ (by Remark 2.6, having no boundary in the interior of $E_{x, l}^{\beta}, C$ would have to coincide with the Special Legendrian 2-sphere tangent to $D_{x}^{l}$ ), which contradicts Lemma 4.4.

So take $p \in \operatorname{spt} C \cap E_{x, l}^{\beta}, p \notin \Sigma_{x}^{l}$. Let $\Sigma_{x}^{P}=\Sigma_{x, p}$ be the 3-surface born at $x$ going through $p$; surely $\widehat{P, D_{0}} \geq \frac{3 \alpha}{4}$. We are going to show now that, up to tilting $\Sigma_{x}^{P}$ a bit, we can assume that it is transversal to $C$ and the intersection is well-defined and non-zero. 
Take $\delta \ll \widehat{D_{x}^{l}, \Sigma_{x, p}}$ and $r \ll \operatorname{dist}(x, p)$ in such a way that (see Figures 4.4 and 4.5)

(iii) $k\left(C\left\llcorner B_{r}(x), \Sigma_{x, p}\right)=Q \quad\right.$ (possible by Remark 3.4, since $\Sigma_{x, p}$ is transversal to $\left.T_{x} C\right)$,

(iv) $C\left\llcorner B_{r}(x) \subset E_{x}^{\delta} \cup\{x\}\right.$ (by Lemma 4.4, with $E_{x}^{\delta}$ denoting the $\delta$-conic neighbourhood of $\left.T_{x} C\right)$.

By homotopy (see the remark following Lemma 3.3)

$$
k\left(C\left\llcorner B_{r}(x), \Sigma_{x}^{Y}\right)=Q\right.
$$

for all but finitely many $Y$ 's in a small ball around $P$ in $\mathbb{C P}^{1}$ (the finitely many $Y$ 's we have to exclude are those that are tangent to the disks $D_{x}^{i}$, so to ensure that $\Sigma_{x}^{Y}$ is transversal to $T_{x} C$ ). The ball should be chosen small enough so that $\widehat{Y, D_{0}} \geq \frac{\alpha}{2}$ and $\Sigma_{x}^{Y}$ stays away from $E_{x}^{\delta}$, so that these $\Sigma_{x}^{Y}$ do not cross $\partial\left(C\left\llcorner B_{r}(x)\right)\right.$, see Figure 4.5 .

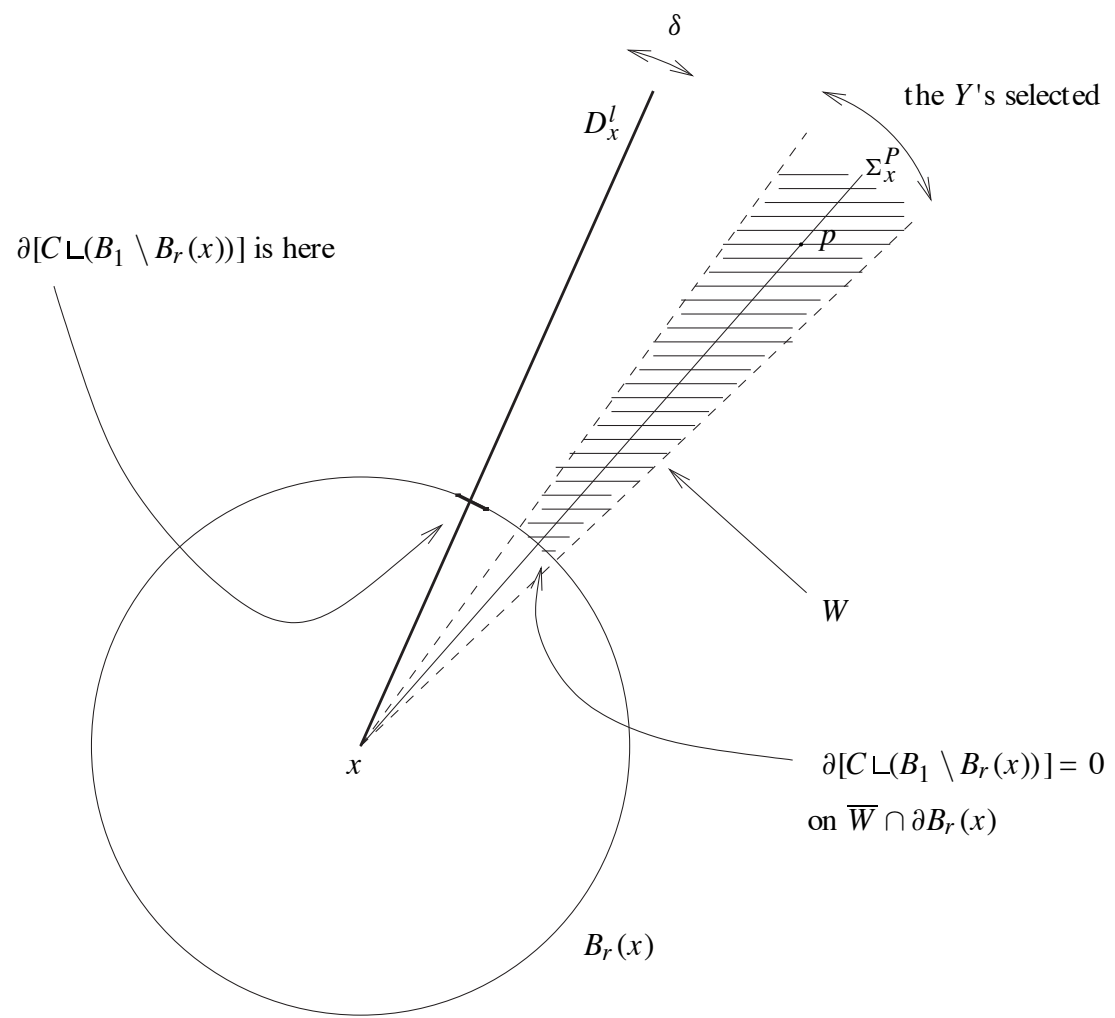

Figure 4.5. Magnify around $x$ : selection of $W$ (shaded region). 
We are going to apply Lemma 4.1:

$$
W=\left(\cup_{Y} \Sigma_{x}^{Y}\right) \cap\left(B_{1}(x) \backslash \overline{B_{r}(x)}\right)
$$

is a foliated neighbourhood of $p$ and we have boundary of $C$ neither on $\bar{W} \cap \partial B_{1}$ (by (i)) nor on $\bar{W} \cap \partial B_{r}(x)$ (by (iv) and by the choice of the $Y$ 's).

So, for the $Y$ 's that we have chosen, if $r$ is small enough, then

$$
k\left(C, \Sigma_{x}^{Y}\right)=k\left(C\left\llcorner B_{r}(x), \Sigma_{x}^{Y}\right)+k\left(C\left\llcorner\left(B_{1} \backslash B_{r}(x)\right), \Sigma_{x}^{Y}\right) \geq Q+1 .\right.\right.
$$

But, by homotopy, going back to the standard notations, we find that $k\left(C_{x_{0}, \rho_{n}}, \Sigma_{x_{n}}^{Y}\right)=$ $k\left(C_{x_{0}, \rho_{n}}, \Sigma_{w}^{Y}\right)$ for some $w \in D_{0} \cap B_{3 / 4}(0)$ (identifying $\mathbb{P} H_{x_{n}}^{4}$ and $\left.\mathbb{P} H_{w}^{4}\right)$. So we have contradicted (ii).

The result just proved will be restated as a relative Lipschitz-type estimate (for the multi-valued graph describing the current) in Corollary 5.8.

\section{Logical structure of the proof of Theorem 1.1 and first part of the inductive step: coordinates, PDEs and average}

Having established the previous results, in this section we start the proof of the regularity Theorem 1.1, which will go on in the next sections.

Structure of the proof. The proof proceeds by induction. By the monotonicity formula, the multiplicity function is upper semi-continuous on the Special Legendrian $C$, therefore the set of points with multiplicity $\geq N$, for $N \in \mathbb{N}$ is closed in $C$. Then, to achieve our result, a singular point $q$ with multiplicity $Q$ being given, we only need to show that singular points of multiplicity $\leq Q$ cannot accumulate onto $q$. The idea is hence to prove this result by induction on the multiplicity $Q$ : at each inductive step, we will assume that we are working in a neighbourhood where $Q$ is the maximal multiplicity.

Basis of induction : $\mathbf{Q}=\mathbf{1}$. We are in an open set where all points of the Special Legendrian $C$ have multiplicity 1 . Since $C$ is minimal $(H=0)$ and boundaryless, we can deduce the smoothness in this set straight from Allard's theorem, see [19]. We can however provide a self-contained argument here: from Theorem 4.7 we know that the tangent planes are continuous, therefore $C$ is a $C^{1}$ current. A classical bootstrapping argument then leads to $C^{\infty}$ regularity.

Assumptions for the inductive step : Q-1 $\Rightarrow \mathbf{Q}$. We are in an open ball $B$, where $\operatorname{Sing} Q$ is a closed set (that could a priori have positive $\mathcal{H}^{2}$ - measure) and $C \backslash \operatorname{Sing} Q$ is smooth except at the points $\operatorname{Sing} \leq Q-1$, which are isolated in the open set $C \backslash \operatorname{Sing} Q$. We are going to divide the proof of the inductive step into two parts:

$\sharp_{1}$ : $\operatorname{Sing}^{Q}$ is made of isolated points in $\mathrm{B}$, i.e. there is no possibility of accumulation of singularities of multiplicity $Q$ to another singularity $p$ of the same multiplicity; 
$\sharp_{2}$ : singularities of multiplicity $\leq Q-1$ cannot accumulate onto a singularity of multiplicity $Q$.

The proof of $\sharp_{1}$ will be achieved in this section and the next: we aim to prove

Theorem 5.1. Let $B^{5}$ be a ball in which the highest multiplicity for the Special Legendrian cycle $C$ is $Q$. Assume that Sing ${ }^{\leq-1}$ is made of isolated points in $\left(C\left\llcorner B^{5}\right) \backslash \operatorname{Sing}^{Q}\right.$. Then the set $\operatorname{Sing}^{Q}$ is made of isolated points in $B^{5}$.

Recall that there is an easy case of $\sharp_{1}$ that we already proved: indeed, for $p \in \operatorname{Sing}^{Q}$ having a tangent cone that is not $Q$ times the same disk, the result is just Theorem 4.6.

Therefore we only need to prove $\sharp_{1}$ if the tangent cone at $p$ is $Q \llbracket D \rrbracket$. As explained in the introduction, Theorem 5.1 will be achieved after having introduced a multi-valued graph that locally describes the Special Legendrian current. In suitable coordinates the branches of the multi-valued graph satisfy the elliptic system of PDEs (1.1), which is a perturbation of the classical Cauchy-Riemann. Thanks to a $W^{1,2}$-regularity result for the average of the multi-valued graph, we will translate the issue of accumulation of singularities of multiplicity $Q$ into a problem of accumulation of zeros for a new multi-valued graph whose branches solve a PDE that is still a perturbation of the classical Cauchy-Riemann. At this stage we will prove 5.1 by a unique continuation argument.

In the present section we provide the fundational steps for the unique continuation argument:

- we find suitable coordinates in which the multi-valued graph satisfies the PDEs (1.1);

- we study the regularity of the average of the multi-valued graph, showing that it is $W^{1,2}$ on $D^{2}$.

The proof of $\sharp_{1}$ will then come to an end in the next section.

Coordinates. We are now going to choose appropriate coordinates to guarantee later a $W^{1,2}$-type estimate. In order to do that, we will need the result contained in the next lemma. First observe the following:

Remark 5.2. Due to the construction of $\Sigma$, given any 3-surface $\Sigma_{q}^{X}$ and for any point $p \in \Sigma_{q}^{X}$, then $T_{p}\left(\Sigma_{q}^{X}\right) \cap H_{p}^{4}$ is a complex line in $H_{p}^{4}$. This can be seen as follows: $T_{p}\left(\Sigma_{q}^{X}\right) \cap H_{p}^{4}$ is a two-dimensional subspace since $\Sigma_{q}^{X}$ is transversal to $H_{p}^{4}$; moreover one of the Special Legendrian spheres foliating (and building up) $\Sigma_{q}^{X}$ must go through $p$ and it is tangent to $H_{p}^{4}$.

Remark 5.3. In the construction of the 3-surfaces $\Sigma_{q}^{X}$ performed in Section 2, $q$ was taken in a neighbourhood of the Special Legendrian 2-sphere $L_{0}$. We can parametrize this neighbourhood of $L_{0}$ with a complex coordinate $w$ such that the point $(1,0,0) \in L_{0}$ has coordinate 0 . By abuse of notation we will also write $\Sigma_{w}^{X}$ instead of $\Sigma_{q}^{X}$ when the point $q \in L_{0}$ has coordinate $w$. 
Lemma 5.4. There exist open neighbourhoods $V, U$ of $[0,1]$ in $\mathbb{C P}^{1}$ so that we can define ${ }^{14}$ the function:

$d: B_{1}^{5} \times V \rightarrow B_{2}^{2} \times U$, given by $d(p, Y)=(w, X)$ s.t. $\Sigma_{w}^{X}$ contains $p$ and $Y \subset T_{p} \Sigma_{w}^{X}$. Moreover, $d$ is of class $C^{1}$.

In other words, for any point $p \in B_{1}^{5}$ and any almost vertical direction $Y$ there exist a unique point $w \in L_{0}$ and direction $X$ such that $\Sigma_{w}^{X}$ goes through $p$ with direction $Y$. Moreover this correspondence is $C^{1}$.

Proof of Lemma 5.4. Take the following neighbourhood $U$ of $[0,1]$ in $\mathbb{C P}^{1}, U=$ $\left\{[Z ; W] \in \mathbb{C P}^{1}:|W|>2|Z|\right\}$. Define the function

$$
\tilde{w}: B_{1}^{5} \times U \rightarrow B_{2}^{2}
$$

where $\tilde{w}=\tilde{w}(p, X)$ is the point in $B_{2}^{2} \cong L_{0} \cap B_{2}^{5}$ such that $p \in \Sigma_{\tilde{w}}^{X}$ ( $\tilde{w}$ is uniquely defined since $\left\{\Sigma_{w}^{X}\right\}$ foliates $B_{1}^{5}$ as the base point runs over $\left.L_{0}\right)$. $\tilde{w}$ is a smooth function.

Recall Remark 5.2. Denote by $\tilde{X}=\tilde{X}(p, X) \in \mathbb{C P}^{1}$ the complex line ${ }^{15}$ in $H_{p}^{4}$ such that $\tilde{X}$, as a 2-dimensional plane, is contained in the tangent to $\Sigma_{\tilde{w}}^{X}$ at $p$. $\tilde{X}(p, X)$ is a smooth perturbation of $X$, since the contact structure in $B_{1}^{5}$ is a smooth perturbation of the integrable structure $\mathbb{C}^{2} \times \mathbb{R}$. Consider

$$
\begin{gathered}
D: B_{1}^{5} \times U \times B_{2}^{2} \times U \rightarrow \mathbb{C} \times \mathbb{C P}^{1} \\
D:(p, Y, w, X) \rightarrow(w-\tilde{w}(p, X), \tilde{X}(p, X)-Y) .
\end{gathered}
$$

The function $D$ is $C^{1}$ and we can compute its $(w, X)$-differential

$$
\frac{\partial D}{\partial(w, X)}=\left(\begin{array}{cc}
1 & \frac{\partial \tilde{w}}{\partial X} \\
0 & \frac{\partial \tilde{X}}{\partial X} \approx 1
\end{array}\right)
$$

and its determinant is non-zero, therefore, by the implicit function theorem, the set $\{D=0\}$ can be described as a graph over $B_{1}^{5} \times V$

$$
(p, Y, d(p, Y))
$$

for some $d \in C^{1}$ and $V \subset U$. The condition $D(p, Y, w, X)=0$ expresses the fact that $\Sigma_{w}^{X}$ goes through $p$ with direction $Y$, thus $d$ satisfies the statement of Lemma 5.4.

14 By $B_{1}^{5}$ we mean the 5-dimensional ball of radius 1 . Analogously for $B_{2}^{2}$, which we implicitly identify with the disk in $\mathbb{C}$ of radius 2 .

${ }^{15}$ Recall that $\mathbb{C P}^{1} \cong \mathbb{P} H_{p}^{4}$. 
Before starting the proof of non-accumulation of singularities of order $Q$ to a singular point $x_{0}$ having tangent cone of the form $Q \llbracket D \rrbracket$, we are going to set coordinates so that the current and the leaves of the chosen foliation $\Sigma^{X}$ have only isolated and at most countably many points of non-transversality.

Recall that a parallel foliation $\left\{\Sigma_{p}^{X}\right\}$ for $X$ fixed, of the type constructed in Section 2 , locally induces a system of 5 real coordinates around $x_{0}=(1,0,0)$, the first two, $(s, t)$, lying in the space of parameters $L_{0}$ (the chosen Special Legendrian 2 -sphere) and the remaining three in $\Sigma$, see Lemma 2.3 and the discussion about families of parallel foliations. We can also think of having a complex coordinate on $L_{0} \cap B_{2}^{5} \cong B_{2}^{2}$ rather than two real ones. This means, for instance, that in this coordinates, if $q \in L_{0}$ has coordinate $z_{0} \in \mathbb{C}$, the leaf $\Sigma_{q}^{X}$ is described by $\left\{\left(z_{0}, b, c, a\right)\right\}$, as $(b, c, a)$ describes to $B_{2}^{3} \subset \mathbb{R}^{3}$. In the same vein, $L_{0}$ is described by $\{(z, 0,0,0)\}$ or by $\{(s, t, 0,0,0)\}$, where we used respectively a complex and two real coordinates for $L_{0} \cap B_{2}^{5} \cong B_{2}^{2}$.

In the coordinates so induced by $\left\{\Sigma_{p}^{X}\right\}$, introduce the projection map $\pi: B_{2}^{2} \times$ $B_{2}^{3} \rightarrow B_{2}^{2}$ sending $(z, b, c, a)$ to $z$.

Now we want to choose a privileged direction $X$ to ensure the transversality announced above. Recall that we are working in a neighbourhood of $x_{0}$ where the multiplicity is everywhere $\leq Q$. Start with coordinates set in such a way that $x_{0}=$ $0, D=D_{0} \cong[1,0]$ and the foliation we are using is given by $\left\{\Sigma_{p}^{[0,1]}\right\}$, and assume that we have blown up enough in order to ensure that spt $\left(C_{0, r}\left\llcorner B_{1}\right) \subset E^{\delta} \cup\{0\}\right.$ for some small $\delta$ (Lemma 4.4) and that $T_{y} C_{0, r}$ makes an angle smaller than $\delta$ for any $y \in \operatorname{Sing}^{Q}$ (Theorem 4.7).

Recall Lemma 5.4 and let $S$ be the smooth part of the current $C_{0, r}$ where the tangent planes are in $V$. Denote by $\pi_{2}$ the projection $\pi_{2}: B_{2}^{2} \times U \rightarrow U$. Define the following function $\psi: S \rightarrow \mathbb{C P}^{1}$

$$
\psi(p):=\pi_{2}\left(d\left(p, T_{p} S\right)\right)
$$

The tangent on $S$ is a smooth function, thus, by composition, $\psi$ is also smooth. Therefore we can find a regular value $X$ for $\psi$ as close as we want to $[0,1]$. We choose then the coordinates induced by this $\Sigma^{X}$, which we will denote by $\{(z, b, c, \bar{a})\}$ or by $\{(s, t, b, c, a)\}$, where $z=s+i t$. They have the property that the leaves $\Sigma_{z}^{X}$ are tangent to the smooth part of the current only at isolated points $\left\{t_{i}\right\}_{i=1}^{\infty}$ (they can possibly accumulate on the singular set). As for the singular set, the points of multiplicity up to $Q-1$ are also isolated singularities by inductive assumption, so we can assume that there is transversality there up to picking a new $X$, again among the regular values (only a countable set of $X$ must be avoided). On the set Sing $Q$ the tangent cone makes a small angle with the horizontal, thanks to the Lipschitz estimate from Theorem 4.7.

Multi-valued graph. With the coordinates just taken, denote by $\pi$ the projection onto $D_{0} \cong\{(z, 0,0)\}$. Recall that we are also assuming to have dilated the current 
about 0 of a factor $r$ small enough to ensure that $C_{r}:=C_{0, r}\left\llcorner B_{1}\right.$ has support $\delta$-close to $T_{0} C$ and that $T_{y} C_{r}$ makes an angle smaller than $\delta$ with $T_{0} C$ for any $y \in \operatorname{Sing} Q$.

We can now say that, by intersection theory, except on the countable set $\left\{\pi\left(t_{i}\right)\right\}$, the leaves intersect $C$ transversally and positively; as explained in Remark 3.4, for some $R<1, \Sigma_{z}^{X}$ intersect the current at exactly $Q$ points (counted with multiplicities) for a.e. $|z|<R$. We have thus defined a $Q$-valued function

$$
\begin{gathered}
\left\{b_{i}, c_{i}, \alpha_{i}\right\}_{i=1}^{Q}(z): D_{R} \rightarrow \mathbb{R}^{3}, \text { or } \\
\left\{\varphi_{i}, \alpha_{i}\right\}_{i=1}^{Q}(z): D_{R} \rightarrow \mathbb{C} \times \mathbb{R},
\end{gathered}
$$

with $D_{R}=\{(z, 0,0),|z|<R\}, \varphi_{j}=b_{j}+i c_{j}$. Equivalently, we have a function from $D_{R}$ into the Q-th symmetric product

$$
\mathcal{S}^{Q}(\mathbb{C} \times \mathbb{R})=\frac{(\mathbb{C} \times \mathbb{R})^{Q}}{\sim}
$$

where two $Q$-tuples are equivalent if one is a permutation of the other. When using the notation $\left\{\varphi_{i}, \alpha_{i}\right\}_{i=1}^{Q}$ it should be kept in mind that the $Q$-tuples are unordered, so the indexation is not global on $D_{R}$.

The $Q$-valued function just constructed is $L^{\infty}$ since the current is contained in a cone $E^{2 \delta}$ around $D_{R}$.

Remark 5.5. Introduce the following notation:

$$
\mathcal{A}=D_{R} \backslash \pi\left(\operatorname{Sing}^{Q}\right), \mathcal{B}=\mathcal{A} \backslash \pi\left(\operatorname{Sing}^{\leq Q-1}\right), \mathcal{G}=\mathcal{B} \backslash \cup_{i=1}^{\infty}\left\{\pi\left(t_{i}\right)\right\} .
$$

$\pi(\operatorname{Sing} Q)$ is a closed set since we are working in a neighbourhood where $Q$ is the highest multiplicity and thanks to the inductive hypotesis, therefore $\mathcal{A}$ is open. $\mathcal{B}=D_{R} \backslash \pi(\operatorname{Sing} \leq Q)$ is also open since $\operatorname{Sing} \leq Q$ is a closed set. $\mathcal{G}$ is open since we are taking away from the open set $\mathcal{B}$ a countable set of isolated points that can only accumulate on the complement of $\mathcal{B}$.

Observe that, locally on $\mathcal{B}$, it is possible to give a coherent global indexation of $\left\{\varphi_{i}, \alpha_{i}\right\}_{i=1}^{Q}$; i.e., for any point in $\mathcal{B}$ there is a small ball centered at this point on which the multifunction is made of $Q$ distinct smooth functions.

Average. Define the average of the branches $\left\{\varphi_{i}, \alpha_{i}\right\}_{i=1}^{Q}$ by

$$
\tilde{\Psi}=(\tilde{\varphi}, \tilde{\alpha}):=\left(\frac{\sum_{i=1}^{Q} \varphi_{i}}{Q}, \frac{\sum_{i=1}^{Q} \alpha_{i}}{Q}\right),
$$

which is a single-valued $L^{\infty}$ function on $D_{R}$. The next steps aim to prove that this average is actually a $W^{1,2}$ function. This will be achieved with Theorem 5.9. The strategy is as follows:

- after writing the PDEs satisfied by the branches of the $Q$-valued function at smooth points, we will estimate that the $W^{1,2}$-norm on $\mathcal{G}$ is finite and bounded by the mass of the current; 
- we will successively extend the estimate to $\mathcal{B}$ and $\mathcal{A}$ by using the fact that, in dimension two, the $W^{1,2}$-capacity of an isolated point is zero;

- eventually, thanks to Theorem 4.7, we will conclude that $(\tilde{\varphi}, \tilde{\alpha})$ is $W^{1,2}$ on the whole of $D_{R}$.

PDEs. As noted above, on the open set $\mathcal{G}$ the branches $\left\{\varphi_{i}, \alpha_{i}\right\}_{i=1}^{Q}$ are locally smooth functions. We restrict ourselves to a small ball $\Delta \subset \mathcal{G}$ on which they can be globally indexed and we are going to write the PDEs satisfied by these $Q$ functions coming from the fact that these (smooth) pieces are calibrated by $\omega$. Notice that also the derivatives of the $Q$ branches are well-defined functions. We are using coordinates $(z, \zeta, a)=(s, t, b, c, a)$, where $z=s+i t, \zeta=b+i c$ are complex and the others real. Recall that $\Sigma$ were built so that the coordinate vectors $\frac{\partial}{\partial b}$ and $\frac{\partial}{\partial c}$ are always tangent to the 4-planes $H^{4}$ of the horizontal distribution. Denote by $J$ the J-structure defined on these hyperplanes,

$$
J_{p}: H_{p}^{4} \rightarrow H_{p}^{4}
$$

We can assume that each leaf $\Sigma_{z}$ is parametrized in such a way that

$$
J\left(\frac{\partial}{\partial b}\right)=\frac{\partial}{\partial c}, \quad J\left(\frac{\partial}{\partial c}\right)=-\frac{\partial}{\partial b} .
$$

Recall that we are assuming, without loss of generality, that the origin of $D_{R} \times \mathbb{R}^{3}$ corresponds to the point $(1,0,0) \in \mathbb{C}^{3}$ (this can be done by rotating $S^{5}$ via a rotation in $S U(3))$. We also assume that $(s, t)$ are such that $\frac{\partial}{\partial s}$ and $\frac{\partial}{\partial t}$ coincide respectively with $\frac{\partial}{\partial x^{2}}$ and $\frac{\partial}{\partial x^{3}}$ in $\mathbb{C}^{3}$ at the point 0 , so $\omega(0)=d x^{2} \wedge d x^{3}-d y^{2} \wedge d y^{3}$ as a form in $\mathbb{C}^{3}$ is $d s \wedge d t+d b \wedge d c$ in the new coordinates. Moreover,

$$
\frac{\partial}{\partial b}, \frac{\partial}{\partial c}, \frac{\partial}{\partial a} \text { are always orthogonal to each other, }
$$

$$
\frac{\partial}{\partial b}, \frac{\partial}{\partial c} \text { are also orthogonal to the unit fiber vector } v\left(v=i \frac{\partial}{\partial r} \text { in } \mathbb{C}^{3}\right) .
$$

All the other scalar products of the $\mathrm{e}^{16}$ coordinate vectors $\frac{\partial}{\partial s}, \frac{\partial}{\partial t}, \frac{\partial}{\partial b}, \frac{\partial}{\partial c}, \frac{\partial}{\partial a}$ at a point $p$ are bounded by $K \varepsilon$, for an arbitrarily small $\varepsilon$, as long as we blow-up of a factor $r$ small enough, since they are orthogonal at the point 0 and the structure is smooth.

Analogously, since the fiber vector at 0 is also equal to $\frac{\partial}{\partial a}$ and orthogonal to $\frac{\partial}{\partial s}, \frac{\partial}{\partial t}$, we have

$$
-K \varepsilon \leq\left\langle\frac{\partial}{\partial s}, v\right\rangle,\left\langle\frac{\partial}{\partial t}, v\right\rangle,\left\langle\frac{\partial}{\partial s}, \frac{\partial}{\partial a}\right\rangle,\left\langle\frac{\partial}{\partial t}, \frac{\partial}{\partial a}\right\rangle \leq K \varepsilon .
$$

16 Throughout the section, $K$ will always represent a constant independent of the chosen $\Delta \subset$ $D_{R}$. 
Further, with $\omega_{r}:=\frac{1}{r^{2}}\left((r x)^{*}(\omega)\right)$, for any $l$ we have that $\left\|\omega_{r}-\omega(0)\right\|_{C^{l}\left(B_{1}\right)} \rightarrow 0$ as $r \rightarrow 0$. Remark that $\omega_{r}$ calibrates the blown-up current $C_{r}$.

As we said before, each branch $\Psi_{j}=\left(\varphi_{j}, \alpha_{j}\right)$ is a well-defined graph on $\Delta$. We can focus on one precise branch, for a certain $j \in\{1, \ldots, Q\}$ : the parametrization of this smooth piece is

$$
\Lambda_{j}(s, t):=\left(s, t, b_{j}(s, t), c_{j}(s, t), \alpha_{j}(s, t)\right),
$$

with tangent vectors

$$
\frac{\partial \Lambda_{j}}{\partial s}=\left(1,0, \frac{\partial b_{j}}{\partial s}, \frac{\partial c_{j}}{\partial s}, \frac{\partial \alpha_{j}}{\partial s}\right), \quad \frac{\partial \Lambda_{j}}{\partial t}=\left(0,1, \frac{\partial b_{j}}{\partial t}, \frac{\partial c_{j}}{\partial t}, \frac{\partial \alpha_{j}}{\partial t}\right) .
$$

On each tangent space $T_{p} S^{5}$ extend $J$ to a linear map defined on the whole of $T_{p} S^{5}$

$$
J: T_{p} S^{5} \rightarrow T_{p} S^{5}
$$

by setting $J\left(\frac{\partial}{\partial a}\right)=\frac{\partial}{\partial a}$ (this is quite arbitrary). Introduce the following notation for the coefficient of this map in the given basis:

$$
J\left(\frac{\partial}{\partial s}\right)=\varsigma \frac{\partial}{\partial s}+\lambda \frac{\partial}{\partial t}+\eta \frac{\partial}{\partial a}+\beta \frac{\partial}{\partial b}+\gamma \frac{\partial}{\partial c},
$$

where $\varsigma, \eta, \beta, \gamma$ are small in modulus, say less than some $K \cdot r$ since they are equal to 0 at the point 0 , while $|\lambda|$ is close to 1 . These five functions depend on the variables $(s, t, b, c, a)$, but we will not explicitly write this dependence. For the other coefficients of $J$, recall (5.1) and the extension of $J$ done above. The condition of being a Special Legendrian expressed by proposition 2.1 is then given by the two relations valid at any point:

$$
\begin{gathered}
\frac{\partial \Lambda_{j}}{\partial s} \wedge \frac{\partial \Lambda_{j}}{\partial t} \subset H^{4}, \\
J\left(\frac{\partial \Lambda_{j}}{\partial s}\right)=\lambda \frac{\partial \Lambda_{j}}{\partial t}+\varsigma \frac{\partial \Lambda_{j}}{\partial s} .
\end{gathered}
$$

The fact that the last two coefficients must be exactly $\lambda$ and $\varsigma$ will be clear in a moment. We explicit now (5.5), using (5.3):

$$
\begin{aligned}
J\left(\frac{\partial \Lambda_{j}}{\partial s}\right)= & \varsigma \frac{\partial}{\partial s}+\lambda \frac{\partial}{\partial t}+\eta \frac{\partial}{\partial a}+\beta \frac{\partial}{\partial b}+\gamma \frac{\partial}{\partial c} \\
& +\frac{\partial b_{j}}{\partial s} \frac{\partial}{\partial c}-\frac{\partial c_{j}}{\partial s} \frac{\partial}{\partial b}+\frac{\partial \alpha_{j}}{\partial s} \frac{\partial}{\partial a} \\
= & \lambda \frac{\partial \Lambda_{j}}{\partial t}+\varsigma \frac{\partial \Lambda_{j}}{\partial s} \\
= & \lambda\left(\frac{\partial}{\partial t}+\frac{\partial b_{j}}{\partial t} \frac{\partial}{\partial b}+\frac{\partial c_{j}}{\partial t} \frac{\partial}{\partial c}+\frac{\partial \alpha_{j}}{\partial t} \frac{\partial}{\partial a}\right) \\
& +\varsigma\left(\frac{\partial}{\partial s}+\frac{\partial b_{j}}{\partial s} \frac{\partial}{\partial b}+\frac{\partial c_{j}}{\partial s} \frac{\partial}{\partial c}+\frac{\partial \alpha_{j}}{\partial s} \frac{\partial}{\partial a}\right)
\end{aligned}
$$


(from comparing the coefficients of $\frac{\partial}{\partial s}$ and $\frac{\partial}{\partial t}$ we can see why we needed $\lambda$ and $\zeta$ in (5.5)). Identifying the coefficients of the coordinate vectors $\frac{\partial}{\partial b}$ and $\frac{\partial}{\partial c}$ in the first and third line of (5.6) leads to

$$
\left\{\begin{array}{c}
-\frac{\partial c_{j}}{\partial s}+\beta=\lambda \frac{\partial b_{j}}{\partial t}+\varsigma \frac{\partial b_{j}}{\partial s} \\
\frac{\partial b_{j}}{\partial s}+\gamma=\lambda \frac{\partial c_{j}}{\partial t}+\varsigma \frac{\partial c_{j}}{\partial s} .
\end{array}\right.
$$

Substituting the expression for $\frac{\partial c_{j}}{\partial s}$ given by the first line of (5.7) into the second we get

$$
\frac{\partial b_{j}}{\partial s}=\lambda \frac{\partial c_{j}}{\partial t}+\varsigma\left(\beta-\lambda \frac{\partial b_{j}}{\partial t}-\varsigma \frac{\partial b_{j}}{\partial s}\right)-\gamma
$$

which implies

$$
\frac{\partial b_{j}}{\partial s}=\frac{\lambda}{1+\varsigma^{2}}\left(\frac{\partial c_{j}}{\partial t}-\varsigma \frac{\partial b_{j}}{\partial t}+\frac{\varsigma \beta-\gamma}{\lambda}\right) .
$$

Plugging this back into the first identity of (5.7) we get

$$
\frac{\partial c_{j}}{\partial s}=-\frac{\lambda}{1+\varsigma^{2}}\left(\frac{\partial b_{j}}{\partial t}+\varsigma \frac{\partial c_{j}}{\partial t}-\frac{\beta+\varsigma \gamma}{\lambda}\right) .
$$

Let us now draw some conclusions from (5.4). We have to impose that $\frac{\partial \Lambda_{j}}{\partial s}$ and $\frac{\partial \Lambda_{j}}{\partial t}$ are always orthogonal to the vertical fiber vector $v$. Since the first two components of $\frac{\partial \Lambda_{j}}{\partial s}$ are fixed and equal $(1,0)$ and $\frac{\partial}{\partial b}, \frac{\partial}{\partial c}$ are orthogonal to $v,(5.4)$ means

$$
\left\langle\frac{\partial}{\partial s}, v\right\rangle=-\frac{\partial \alpha_{j}}{\partial s}\left\langle\frac{\partial}{\partial a}, v\right\rangle
$$

Doing the same with $\frac{\partial \Lambda_{j}}{\partial t}$ we obtain

$$
\left\langle\frac{\partial}{\partial t}, v\right\rangle=-\frac{\partial \alpha_{j}}{\partial t}\left\langle\frac{\partial}{\partial a}, v\right\rangle
$$

Since $\left\langle\frac{\partial}{\partial a}, v\right\rangle$ is close to 1 (see (5.2)), we get

$$
\left|\frac{\partial \alpha_{j}}{\partial s}\right|,\left|\frac{\partial \alpha_{j}}{\partial t}\right| \leq K \varepsilon
$$


We can rewrite ${ }^{17}$ equations (5.8), (5.9), (5.10) and (5.11) as

$$
\left\{\begin{array}{l}
\frac{\partial b_{j}}{\partial s}=A \frac{\partial c_{j}}{\partial t}+B \frac{\partial b_{j}}{\partial t}+C \\
\frac{\partial c_{j}}{\partial s}=-A \frac{\partial b_{j}}{\partial t}+B \frac{\partial c_{j}}{\partial t}+F \\
\nabla \alpha_{j}=h\left(s, t, \Psi_{j}\right)
\end{array}\right.
$$

Here $A, B, C, F$ are smooth real functions of $\left(s, t, b_{j}(s, t), c_{j}(s, t), \alpha_{j}(s, t)\right)$ with $A(0,0,0,0,0)=1, B(0,0,0,0,0)=C(0,0,0,0,0)=F(0,0,0,0,0)=0$, so $A$ is close to 1 and $B, C, F$ are less than $\varepsilon$ in modulus ${ }^{18}$. The $\mathbb{R}^{2}$-valued function $h$ is Lipschitz thanks to (5.12).

Complex PDE. We are going to rewrite the first two equations in (5.13) in complex form, so we use the complex coordinate $z=s+i$, and observe the function $\varphi_{j}(z)=b_{j}(s, t)+i c_{j}(s, t)$. The complex derivatives $\frac{\partial}{\partial z}=\frac{1}{\sqrt{2}}\left(\frac{\partial}{\partial s}-i \frac{\partial}{\partial t}\right)$ and $\frac{\partial}{\partial \bar{z}}=\frac{1}{\sqrt{2}}\left(\frac{\partial}{\partial s}+i \frac{\partial}{\partial t}\right)$ will be denoted respectively by $\partial$ and $\bar{\partial}$. Compute the first equation in (5.13) plus $i$ times the second:

$$
\frac{\partial \varphi_{j}}{\partial s}=(-i A+B) \frac{\partial \varphi_{j}}{\partial t}+C+i F .
$$

Then

$$
\left\{\begin{array}{c}
\sqrt{2} \bar{\partial} \varphi_{j}=((1-A) i+B) \frac{\partial \varphi_{j}}{\partial t}+C+i F, \\
\sqrt{2} \partial \varphi_{j}=(-(1+A) i+B) \frac{\partial \varphi_{j}}{\partial t}+C+i F .
\end{array}\right.
$$

We seek a function $v=v_{1}+i v_{2}$ so that

$$
(1-A) i+B=-\left(v_{1}+i v_{2}\right)(-(1+A) i+B),
$$

which rewrites, separating imaginary and real parts:

$$
\left(\begin{array}{cc}
1+A & -B \\
B & 1+A
\end{array}\right)\left(\begin{array}{l}
v_{1} \\
\nu_{2}
\end{array}\right)=\left(\begin{array}{c}
1-A \\
-B
\end{array}\right) .
$$

The matrix on the 1.h.s. is a perturbation of $2 I d$, and the vector on the r.h.s. has norm bounded by $\varepsilon$, therefore we can invert the system and find that there is

17 Recall again that we are focusing on a chosen branch $\Psi_{j}=\left(b_{j}, c_{j}, \alpha_{j}\right)$, which describes a smooth piece of the multi-valued graph above $\Delta$.

$18 \varepsilon$ is a positive number which can be assumed as small as we wish: it is of order $r$, the rescaling factor that we used for the blow-up. 
a unique solution for $v=v_{1}+i v_{2}$ whose norm is bounded by $\varepsilon$. Then, setting $\mu=\frac{1}{\sqrt{2}}(1+v)(C+i F)$ we can write, from (5.14),

$$
\bar{\partial} \varphi_{j}+v\left(z, \varphi_{j}, \alpha_{j}\right) \partial \varphi_{j}+\mu\left(z, \varphi_{j}, \alpha_{j}\right)=0,
$$

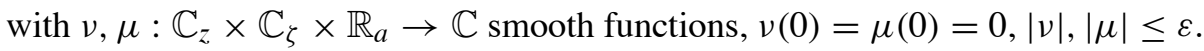

The first two equations in (5.13), or equivalently equation (5.15), are perturbations of the classical Cauchy-Riemann equations. Notice however that the coefficients depend on $s, t, b_{j}(s, t), c_{j}(s, t)$ and $\alpha_{j}(s, t)$, and we need the third equation in (5.13), to clarify the " $\alpha$-dependence".

At this stage, we can estimate the $L^{2}$-norm of the jacobian of $\Psi_{j}$ using (5.8), (5.9) and (5.12). Recall that the functions $\varsigma, \eta, \beta, \gamma$ are in modulus smaller than $K \varepsilon$ and $\lambda$ is close to 1 . The metrics in the base space $\Delta_{s, t}$ and in the target $\mathbb{R}_{b, c, a}^{3}$ are perturbation of the standard Euclidean metrics (at 0 they coincide with them), so

$$
\begin{aligned}
\left|D \Psi_{j}\right|^{2} & \leq K\left(\left|\frac{\partial b_{j}}{\partial s}\right|^{2}+\left|\frac{\partial b_{j}}{\partial t}\right|^{2}+\left|\frac{\partial c_{j}}{\partial s}\right|^{2}+\left|\frac{\partial c_{j}}{\partial t}\right|^{2}+\left|\frac{\partial \alpha_{j}}{\partial s}\right|^{2}+\left|\frac{\partial \alpha_{j}}{\partial t}\right|^{2}\right) \\
& \leq K\left(\left|\frac{\partial b_{j}}{\partial t}\right|^{2}+\left|\frac{\partial c_{j}}{\partial t}\right|^{2}+C \varepsilon^{2}\right)+K \varepsilon^{2} \\
& \leq K\left(1+\left|\frac{\partial b_{j}}{\partial t}\right|^{2}+\left|\frac{\partial c_{j}}{\partial t}\right|^{2}\right) .
\end{aligned}
$$

The constant $K$ obtained at the end only depends on the factor $r$ that we used for the dilation and is valid for any smaller $r$, moreover it is independent of the chosen $\Delta$. We can assume that $K=2$, since this constant gets closer to 1 as $r \rightarrow 0$.

$W^{\mathbf{1}, 2}$ estimate for the average. For $\Psi_{j}=\left(b_{j}, c_{j}, \alpha_{j}\right)$ (we are still focusing, locally on $\Delta$, on a single smooth branch), consider $\left(\Psi_{j}\right)^{*} \omega_{0}=\left(1+\frac{\partial b_{j}}{\partial s} \frac{\partial c_{j}}{\partial t}-\frac{\partial b_{j}}{\partial t} \frac{\partial c_{j}}{\partial s}\right) d s \wedge$ $d t$ and plug in (5.8) and (5.9):

$$
\begin{aligned}
\left(\Psi_{j}\right)^{*} \omega_{0} \geq & 1+\frac{\lambda}{1+\varsigma^{2}}\left(\frac{\partial c_{j}}{\partial t}\right)^{2}+\frac{\lambda}{1+\varsigma^{2}}\left(\frac{\partial b_{j}}{\partial t}\right)^{2} \\
& -\varepsilon\left|\frac{\partial b_{j}}{\partial t} \frac{\partial c_{j}}{\partial t}\right|-\varepsilon\left|\frac{\partial b_{j}}{\partial t}\right|-\varepsilon\left|\frac{\partial c_{j}}{\partial t}\right| \\
\geq & \frac{1}{2}\left(1+\left|\frac{\partial b_{j}}{\partial t}\right|^{2}+\left|\frac{\partial c_{j}}{\partial t}\right|^{2}\right) \geq \frac{1}{4}\left(1+\left|\nabla b_{j}\right|^{2}+\left|\nabla c_{j}\right|^{2}\right),
\end{aligned}
$$

where we used $\varepsilon\left|\frac{\partial b_{j}}{\partial t} \frac{\partial c_{j}}{\partial t}\right| \leq \frac{1}{2}\left(\varepsilon\left(\frac{\partial b_{j}}{\partial t}\right)^{2}+\varepsilon\left(\frac{\partial c_{j}}{\partial t}\right)^{2}\right)$ and $\varepsilon\left|\frac{\partial c_{j}}{\partial t}\right| \leq \frac{1}{2}\left(\varepsilon+\varepsilon\left(\frac{\partial c_{j}}{\partial t}\right)^{2}\right)$, the hypothesis on $\varsigma, \eta, \beta, \gamma, \lambda$ and (5.16) with $K=2$ as said above. 
Consider now $\omega_{r}-\omega_{0}$. Write this 2-form in the canonical basis in the coordinates $s, t, b, c, a$. All the coefficients are smaller than $\varepsilon$ in modulus, if $r$ was chosen small enough. Therefore

$$
\left(\Psi_{j}\right)^{*}\left(\omega_{r}-\omega_{0}\right)
$$

is a 2-form in $d s \wedge d t$ whose coefficient comes from summing products of derivatives of $\Psi_{j}$. As above, we can bound this coefficient by $\varepsilon\left(1+\left|\frac{\partial b_{j}}{\partial t}\right|^{2}+\left|\frac{\partial c_{j}}{\partial t}\right|^{2}\right)$. Using this fact, together with (5.17) and the triangle inequality we have

$$
\int_{\Delta}\left(\Psi_{j}\right)^{*} \omega_{r} \geq\left(\frac{1}{4}-\varepsilon\right) \int_{\Delta} 1+\left|\nabla b_{j}\right|^{2}+\left|\nabla c_{j}\right|^{2} .
$$

Recalling (5.16) we can finally write the desired estimate:

$$
\int_{\Delta}\left|D \Psi_{j}\right|^{2} \leq K \int_{\Delta}\left(\Psi_{j}\right)^{*} \omega_{r}=K \int_{\Psi_{j}(\Delta)} \omega_{r}=K \cdot \mathcal{H}^{2}\left(\Psi_{j}(\Delta)\right),
$$

with a constant $K$ independent of the chosen $\Delta$. We can therefore conclude, recalling the notations taken during the inductive assumptions,

Lemma 5.6. On the $\operatorname{set} \mathcal{G}=\left(D_{R} \backslash \operatorname{Sing} \leq Q\right) \backslash \cup_{i=1}^{\infty}\left\{\pi\left(t_{i}\right)\right\}$ it holds

$$
\sum_{i=1}^{Q} \int_{\mathcal{G}}\left(\left|D \varphi_{i}\right|^{2}+\left|D \alpha_{i}\right|^{2}\right) \leq K \cdot \mathcal{H}^{2}\left(C_{r}\right)<\infty
$$

and therefore the average function $\tilde{\Psi}=(\tilde{\varphi}, \tilde{\alpha})$ is $W^{1,2}(\mathcal{G})$ with norm bounded by the mass of $C_{r}$ (we already knew that it is $L^{\infty}$ ).

The next considerations will allow us to extend this estimate for $(\tilde{\varphi}, \tilde{\alpha})$ to the set $\mathcal{B}=\mathcal{G} \cup_{i=1}^{\infty}\left\{\pi\left(t_{i}\right)\right\}$. One can do this in a straightforward way recalling that the capacity of a point in $\mathbb{R}^{2}$ is zero. Anyway we also give a direct proof. Rename for notational convenience $q_{i}=\pi\left(t_{i}\right)$ and take $B_{\rho_{i}}^{2}\left(q_{i}\right) \subset \mathcal{B}$ balls centered at the $q_{i}$ 's so that $\sum_{i} \rho_{i} \leq \delta$, for $\delta$ chosen arbitrarily small. Let $\xi$ be any test-function in $C_{c}^{\infty}(\mathcal{B})$. Then

$$
\begin{aligned}
\left|\int_{\mathcal{B}} \tilde{\varphi} \frac{\partial \xi}{\partial s}\right| & \leq\left|\int_{\cup_{i} B_{\rho_{i}}\left(q_{i}\right)} \tilde{\varphi} \frac{\partial \xi}{\partial s}\right|+\left|\int_{\mathcal{B} \backslash \cup_{i} B_{\rho_{i}}\left(q_{i}\right)} \frac{\partial \tilde{\varphi}}{\partial s} \xi\right|+\sum_{i}\left|\int_{\partial B_{\rho_{i}}\left(q_{i}\right)} \tilde{\varphi} \xi\left\langle\frac{\partial}{\partial s}, v\right\rangle\right| \\
& \leq C \delta^{2}\|\tilde{\varphi}\|_{\infty}\|\nabla \xi\|_{\infty}+\|\tilde{\varphi}\|_{W^{1,2}(\mathcal{G})}\|\xi\|_{L^{2}(\mathcal{B})}+C \delta\|\tilde{\varphi}\|_{\infty}\|\xi\|_{\infty} .
\end{aligned}
$$

Since $\delta$ was arbitrarily small,

$$
\left|\int_{\mathcal{B}} \tilde{\varphi} \frac{\partial \xi}{\partial s}\right| \leq\|\tilde{\varphi}\|_{W^{1,2}(\mathcal{G})}\|\xi\|_{L^{2}(\mathcal{B})} .
$$


We can do the same for the $t$-derivative. For $\tilde{\alpha}$ things are even easier, indeed $\tilde{\alpha}$ is Lipschitz. Therefore the average function is $W^{1,2}$ on $\mathcal{B}$ with the same norm as on $\mathcal{G}$. We can do the same passing from $\mathcal{B}$ to $\mathcal{A}=D_{R} \backslash \operatorname{Sing}^{Q}$ : again we have to add a (countable) set of points which are isolated in $\mathcal{A}$, so the same as above applies. Eventually we have proved

Lemma 5.7. On the set $\mathcal{A}=D_{R} \backslash$ Sing $^{Q}$ the average function $\tilde{\Psi}=(\tilde{\varphi}, \tilde{\alpha})$ defines $a W^{1,2}$ map from $\mathcal{A}$ into $\mathbb{C} \times \mathbb{R}$ with norm bounded by the mass of $C_{r}$.

The next step will establish the definitive result on the whole of $D_{R}$.

The following corollary is basically a restatement of Theorem 4.7 as a relative Lipschitz estimate, in terms of coordinates in which the current is seen as a multi-valued graph:

Corollary 5.8. Let $x_{0} \in \operatorname{Sing} Q$ and $T_{x_{0}} C=Q \llbracket D_{0} \rrbracket$, as before. Take coordinates $D^{2} \times \mathbb{C} \times \mathbb{R}$ so that $x_{0}$ is at the origin and $D_{0}$ is identified with $D^{2} \times\{0\}$.

Then $\forall \varepsilon>0 \exists r=r\left(\varepsilon, x_{0}\right)$ such that

$$
\begin{gathered}
\forall x=(z, \zeta, a) \in \mathcal{C}^{\mathcal{Q}}:=\{p \in \mathcal{C}: \theta(p)=Q\} \text { and } x^{\prime}=\left(z^{\prime}, \zeta^{\prime}, a^{\prime}\right) \in \operatorname{sptC} \cap B_{r}\left(x_{0}\right) \\
\text { we have the estimate }\left|(\zeta, a)-\left(\zeta^{\prime}, a^{\prime}\right)\right|_{\mathbb{C} \times \mathbb{R}} \leq \varepsilon\left|z-z^{\prime}\right|_{\mathbb{R}^{2}} .
\end{gathered}
$$

Proof of Corollary 5.8. The estimate for the third coordinate $a$ is obvious. We need to show that, identifying $\mathbb{C} \equiv \mathbb{R}^{2}$,

$$
\left|\left(\zeta-\zeta^{\prime}\right)\right|_{\mathbb{R}^{2}} \leq \varepsilon\left|z-z^{\prime}\right|_{\mathbb{R}^{2}}
$$

We are going to use Theorem 4.7, which guarantees the continuity at 0 of tangent cones at points in $\mathcal{C}^{\mathcal{Q}}$. Choose $r$ s.t. $\forall x \in B_{2 r}(0)$ having multiplicity $Q$ the angular distance $\widehat{D_{0}, T_{x}} C$ is less than $\frac{\varepsilon}{2}$; we can also guarantee that

$$
k\left(C\left\llcorner B_{2 r}(0), \Sigma_{w}^{X}\right)=Q\right.
$$

for any $w \in D_{0} \cap B_{3 r / 4}(0)$ and $Y \in \mathbb{C P}^{1}$ realizing $\widehat{D_{0}, Y} \geq \varepsilon$. Assume by contradiction that we can find $x \in \mathcal{C}^{\mathcal{Q}}$ and $y \in \operatorname{spt} C$, with $x, y \in B_{r}(0)$ for which

$$
\left|\left(\zeta-\zeta^{\prime}\right)\right|_{\mathbb{R}^{2}}>\varepsilon\left|z-z^{\prime}\right|_{\mathbb{R}^{2}}
$$

holds. Then take $\Sigma_{x, y}$ : this 3 -surface is transversal to the current at $x$ since $T_{x} \widehat{C, \Sigma_{x}, y}>\frac{\varepsilon}{2}$ and we can tilt it a bit finding a $\Sigma_{x}^{Y}$ transversal to $C$, with $\widehat{T_{x} C, Y}>$ $\frac{\varepsilon}{2}$ and with a non-zero intersection, as already done in the proof of Theorem 4.7. Then

$k\left(C\left\llcorner B_{2 r}(0), \Sigma_{x}^{Y}\right)=k\left(C\left\llcorner B_{\rho}(x), \Sigma_{x}^{Y}\right)+k\left(C\left\llcorner\left(B_{2 r}(0)-B_{\rho}(x)\right), \Sigma_{x}^{Y}\right) \geq Q+1\right.\right.\right.$,

for some small enough $\rho \ll \operatorname{dist}(x, y)$. Since $\widehat{D_{0}, Y}>\varepsilon$, we can homotope $\Sigma_{x}^{Y}$ into a $\Sigma_{w}^{Y}$ for some $w \in D_{0} \cap B_{3 r / 4}(0)$ keeping it away from $C$ on $\partial B_{2 r}$, so we are contradicting the identity in (5.19). 


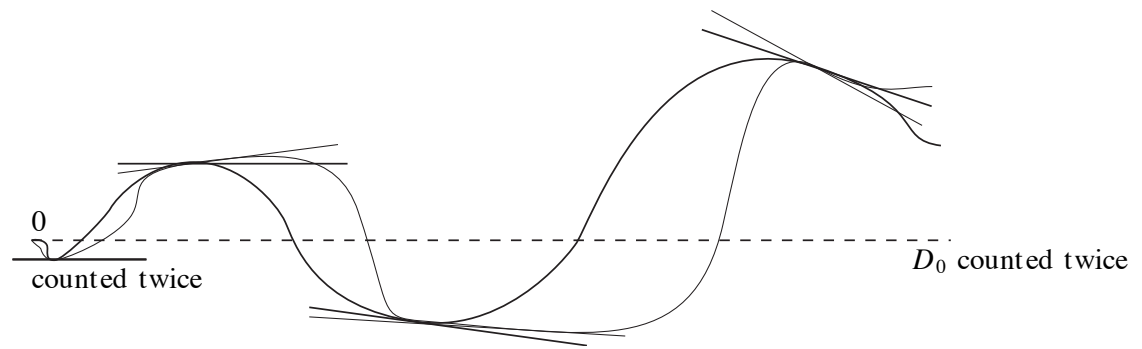

Figure 5.1. An example for $Q=2$, with the current sketched as two curves. The tangents at points of multiplicity 2 flatten as they approach 0 .

Theorem 5.9. The average function $\tilde{\Psi}: D_{R} \rightarrow \mathbb{R}^{3}$ is in $W^{1,2}\left(D_{R}\right)$.

Proof of Theorem 5.9. $\operatorname{Sing}^{Q}$ is a closed set (possibly with positive $\mathcal{H}^{2}$-measure) and on $\mathcal{F}=\pi\left(\operatorname{Sing}^{Q}\right)$ (still a closed set) $\tilde{\Psi}$ coincides with the $Q$ branches $\left\{\Psi_{i}\right\}_{i=1}^{Q}$. We know that the Lipschitz estimate of Corollary 5.8 holds for any couple of points $x, y$ such that $\tilde{\Psi}(x) \in \operatorname{Sing}{ }^{Q}$. In particular, $\left.\tilde{\Psi}\right|_{\mathcal{F}}$ is Lipschitz, it is therefore possible to extend it to a function $u$ defined on the whole of $D_{R}$ which is Lipschitz with constant $K$ equal 3 times the Lipschitz constant of $\left.\tilde{\Psi}\right|_{\mathcal{F}}$ (see [7, Section 2.10.44]). Let $\delta$ be positive and arbitrarily small. Take now a smooth compactly supported function $\sigma_{\delta}$ such that

$$
\sigma_{\delta}(x)= \begin{cases}1 & \text { if } \operatorname{dist}(x, \mathcal{F}) \leq \delta \\ 0 & \text { if } \operatorname{dist}(x, \mathcal{F}) \geq 2 \delta\end{cases}
$$

and $\left|D \sigma_{\delta}\right| \leq \frac{k}{\delta}$ for some $k>0$. Explicitly $\sigma_{\delta}$ can be defined as follows: take a smooth bump-function $\chi$ on $[0, \infty)$, which is 1 on $[0,1)$ and 0 on $[2, \infty)$. Set $\chi_{r, y}(x)=\chi\left(\frac{|x-y|}{r}\right)$ for $x, y \in \mathbb{C}$. Define

$$
\sigma_{\delta}(z)=\frac{G}{\delta^{4}} \int_{\left\{x: \operatorname{dist}(x, \mathcal{F}) \leq \frac{3 \delta}{2}\right\}} \chi_{\frac{\delta}{4}, z}(w) d w d \bar{w},
$$

the right normalization constant $G$ depending on $\int_{0}^{\infty} \chi(t) t^{3} d t$. Introduce

$$
\tilde{\Psi}_{\delta}:=\sigma_{\delta} u+\left(1-\sigma_{\delta}\right) \tilde{\Psi}
$$

and notice that, for any $\delta>0$, this function is $W^{1,2}$. Moreover, for $x \in\{\operatorname{dist}(x, \mathcal{F}) \leq$ $2 \delta\}$, denoting by $p \in \mathcal{F}$ the point realizing this distance, from Corollary 5.8 and by the definition of $u$

$$
|(u-\tilde{\Psi})(x)|=|u(x)-u(p)+\tilde{\Psi}(p)-\tilde{\Psi}(x)| \leq 2 K|p-x| \leq 4 K \delta .
$$

In the lines that follow, $D$ denotes the partial derivative with respect to either of the coordinates $s, t$; notice that, in order to control $D \tilde{\Psi}_{\delta}$, we need to take $D \tilde{\Psi}$ only on 
the set $\{\operatorname{dist}(x, \mathcal{F}) \geq \delta\} \subsetneq \mathcal{A}$, since elsewhere $1-\sigma_{\delta}=0$, so we can freely take derivatives.

$$
\begin{aligned}
D \tilde{\Psi}_{\delta} & =\left(D \sigma_{\delta}\right) u+\sigma_{\delta} D u-\left(D \sigma_{\delta}\right) \tilde{\Psi}+\left(1-\sigma_{\delta}\right) D \tilde{\Psi} \\
& =\left(D \sigma_{\delta}\right)(u-\tilde{\Psi})+\sigma_{\delta} D u+\left(1-\sigma_{\delta}\right) D \tilde{\Psi}
\end{aligned}
$$

We can now compute

$$
\begin{aligned}
\left\|D \tilde{\Psi}_{\delta}\right\|_{L^{2}\left(D_{R}\right)}^{2} \leq & \int_{\{\delta \leq \operatorname{dist}(x, \mathcal{F}) \leq 2 \delta\}}\left|D \sigma_{\delta}\right|^{2}|u-\tilde{\Psi}|^{2}+\int_{D_{R}}\left|\sigma_{\delta}\right|^{2}|D u|^{2} \\
& +\int_{\{\operatorname{dist}(x, \mathcal{F}) \geq \delta\}}\left|1-\sigma_{\delta}\right|^{2}|D \tilde{\Psi}|^{2} \\
\leq & c(K, k)+\|D \tilde{\Psi}\|_{L^{2}(A)}^{2} \leq c(K, k) .
\end{aligned}
$$

So the $W^{1,2}$-norm of the $\tilde{\Psi}_{\delta}$ are uniformly bounded as $\delta \rightarrow 0$, therefore, by compactness, we can find a sequence $\tilde{\Psi}_{\delta_{n}}, \delta_{n} \rightarrow 0$, which converges in $L^{2}$ and weakly* in $W^{1,2}$ to some $\psi \in W^{1,2}\left(D_{R}\right)$. On the other hand, from the computation above,

$$
\left|\tilde{\Psi}_{\delta}-\tilde{\Psi}\right|=\left|\sigma_{\delta}(u-\tilde{\Psi})\right|= \begin{cases}0 & \text { on } \mathcal{F} \\ \leq 4 K \delta & \text { on }\{\operatorname{dist}(x, \mathcal{F}) \leq 2 \delta\}-\mathcal{F} \\ 0 & \text { on }\{\operatorname{dist}(x, \mathcal{F}) \geq 2 \delta\}\end{cases}
$$

so $\tilde{\Psi}_{\delta_{n}}$ converge uniformly to $\tilde{\Psi}$ on $D_{R}$. Therefore $\mathcal{H}^{2}$-a.e. it holds $\psi=\tilde{\Psi}$ and Theorem 5.9 is proven.

\section{End of the proof of $\sharp_{1}$ : unique continuation}

In this section we will complete the proof of $\sharp_{1}$, the first part of the inductive step, i.e. the fact that there is no possibility of accumulation among singularities of equal multiplicity.

Hölder estimate. We are going to establish the following

Theorem 6.1 (Hölder estimate). For any small enough disk $D_{R}$, there exist constants $C, \delta>0$ such that, for any $r \leq R$,

$$
\sum_{j=1}^{Q} \int_{D_{r}}\left|\nabla \varphi_{j}\right|^{2} \leq C r^{\delta}
$$

This easily yields

$$
\int_{D_{r}}|\nabla \tilde{\Psi}|^{2} \leq C r^{\delta}
$$


Remark 6.2. This decay implies that $\tilde{\Psi}$ is $\frac{\delta}{2}$-Hölder thanks to Morrey's embedding theorem, see [15] for instance.

Remark 6.3. The integral in (6.1) should always be understood as

$$
\sum_{j=1}^{Q}\left(\int_{\left(D_{r}-\mathcal{F}\right) \backslash \pi(\operatorname{Sing} \leq Q-1)}\left|d \varphi_{j}\right|^{2} d s d t+\int_{\mathcal{F}}|\nabla \tilde{\varphi}|^{2} \nabla \mathcal{H}^{2}\right),
$$

where $\mathcal{F}=\pi(\operatorname{Sing} Q)$; recall that all branches agree with the average on $\mathcal{F}$ and that Sing $\leq Q-1$ is made of at most countably many points, isolated in $D_{r}-\mathcal{F}$.

Proof of Theorem 6.1. Remark that the $\alpha_{j}$-s are Lipschitz thanks to (5.12); therefore, once (6.1) will be established, (6.2) will follow immediately.

We are going to analyse the behaviour of the function

$$
y(r)=\sum_{j=1}^{Q} \int_{D_{r}}\left|\nabla \varphi_{j}\right|^{2}
$$

Remark that, with our choice of $\partial$ and $\bar{\partial}$, it holds $\left|\nabla \varphi_{j}\right|^{2}=\left|\partial \varphi_{j}\right|^{2}+\left|\bar{\partial} \varphi_{j}\right|^{2}$. We already showed in the previous section that, for any $r$ small enough, $y(r)$ is finite, being bounded by the mass of the current in the cylinder $Z_{r}=D_{r} \times \mathbb{R}^{3}=\{|z| \leq r\}$. Recalling that $C$ is boundaryless,

$$
\left(C\left\llcorner Z_{r}\right)(d \zeta \wedge d \bar{\zeta})=\left(\partial\left(C\left\llcorner Z_{r}\right)\right)\left(\frac{\zeta d \bar{\zeta}-\bar{\zeta} d \zeta}{2}\right)=i\langle C,|z|, r\rangle(\mathcal{I} m(\zeta d \bar{\zeta}))\right.\right.
$$

Denote by $T$ the simple 2-vector describing the oriented approximate tangent plane to the rectifiable set $\mathcal{C}$; by definition

$$
\begin{aligned}
\left(C\left\llcorner Z_{r}\right)\left(\frac{i}{2} d \zeta \wedge d \bar{\zeta}\right)=\right. & \sum_{j=1}^{Q} \int_{\left\{\varphi_{j}, \alpha_{j}\right\}\left(D_{r}\right)} \frac{i}{2}\langle d \zeta \wedge d \bar{\zeta}, T\rangle d \mathcal{H}^{2} \\
= & \sum_{j=1}^{Q} \int_{D_{r}-\mathcal{F}}\left(\left|\partial \varphi_{j}\right|^{2}-\left|\bar{\partial} \varphi_{j}\right|^{2}\right) d s d t \\
& +\sum_{j=1}^{Q} \int_{\text {Sing } Q} \frac{i}{2}\langle d \zeta \wedge d \bar{\zeta}, T\rangle d \mathcal{H}^{2} \\
= & \sum_{j=1}^{Q} \int_{D_{r}-\mathcal{F}}\left(\left|\nabla \varphi_{j}\right|^{2}-2\left|\bar{\partial} \varphi_{j}\right|^{2}\right) d s d t \\
& +\sum_{j=1}^{Q} \int_{\text {Sing } Q} \frac{i}{2}\langle d \zeta \wedge d \bar{\zeta}, T\rangle d \mathcal{H}^{2} .
\end{aligned}
$$


Recalling that the average $\tilde{\varphi}$ is $W^{1,2}$ and that the tangent plane at points in $\operatorname{Sing} Q$ is $Q$ times the tangent to the average, we can rewrite this last term as

$$
\sum_{j=1}^{Q} \int_{D_{r}-\mathcal{F}}\left(\left|\nabla \varphi_{j}\right|^{2}-2\left|\bar{\partial} \varphi_{j}\right|^{2}\right) d s d t+Q \int_{\mathcal{F}}\left(|\nabla \tilde{\varphi}|^{2}-2|\bar{\partial} \tilde{\varphi}|^{2}\right) d \mathcal{H}^{2}
$$

So we have

$$
\begin{aligned}
& \sum_{j=1}^{Q} \int_{D_{r}-\mathcal{F}}\left|\nabla \varphi_{j}\right|^{2} d s d t+Q \int_{\mathcal{F}}|\nabla \tilde{\varphi}|^{2} d \mathcal{H}^{2} \\
& =\sum_{j=1}^{Q} \int_{D_{r}-\mathcal{F}} 2\left|\bar{\partial} \varphi_{j}\right|^{2} d s d t \\
& \quad+Q \int_{\mathcal{F}} 2|\bar{\partial} \tilde{\varphi}|^{2} d \mathcal{H}^{2}+\langle C,|z|, r\rangle\left(-\frac{1}{2} \mathcal{I} m(\zeta d \bar{\zeta})\right) .
\end{aligned}
$$

Now (5.15), which is satisfied by the smooth parts of $\left\{\varphi_{j}\right\}_{j=1}^{Q}$, i.e. on $D_{R} \backslash \mathcal{F}$ minus countably many points, gives

$$
\sum_{j=1}^{Q} \int_{D_{r}-\mathcal{F}}\left|\bar{\partial} \varphi_{j}\right|^{2} d s d t \leq C_{1} \varepsilon^{2} \sum_{j=1}^{Q} \int_{D_{r}-\mathcal{F}}\left|\nabla \varphi_{j}\right|^{2} d s d t+C_{2} r^{2}
$$

Putting (6.3) and (6.4) together,

$$
\begin{aligned}
y(r) & =\sum_{j=1}^{Q}\left(\int_{D_{r}-\mathcal{F}}\left|\nabla \varphi_{j}\right|^{2} d s d t+\int_{\mathcal{F}}|\nabla \tilde{\varphi}|^{2} d \mathcal{H}^{2}\right) \\
& \leq 3 Q \int_{\mathcal{F}}|\nabla \tilde{\varphi}|^{2} d \mathcal{H}^{2}+K_{1}\langle C,|z|, r\rangle\left(-\frac{1}{2} \mathcal{I} m(\zeta d \bar{\zeta})\right)+C_{3} r^{2} .
\end{aligned}
$$

By Corollary $5.8,|\nabla \tilde{\varphi}|$ is bounded by a small constant on $\mathcal{F}$, so

$$
y(r) \leq K_{1}\langle C,|z|, r\rangle\left(-\frac{1}{2} \mathcal{I} m(\zeta d \bar{\zeta})\right)+K_{2} r^{2} .
$$

The slice of the current with $|z|=r$ exists as a rectifiable 1-current for a.e. $r$, as explained in [8, Lemma 1, page 152]. On the set $D_{R} \backslash \mathcal{F}$, the multi-valued graph is smooth except at a countable set of isolated points. For all but countably many choices of $r, \partial D_{r}$ will avoid this set. For a.e. $r$ the current $\langle C,|z|, r\rangle$ is described by the same multi-valued graph $\left\{\varphi_{j}\right\}$. This multi-valued graph, being one-dimensional, can be actually described as a superposition of honest $W^{1,2}$ functions as follows: 
(i) $\partial D_{r} \cap \mathcal{F}=\emptyset$ : for such a $r,\left\{\varphi_{j}\right\}$ is smooth on $\partial D_{r}$, then, starting from any point in the multigraph, we can follow the loop and we will eventually come back to the same point after a certain number $n$ of laps, $n_{1} \leq Q$. Then we can define the function $g_{1}$ to be equal $\varphi_{j}$ on an interval $I_{1}$ of length $2 \pi n_{1} r$, and $g_{1}$ has the same value at the endpoints of $I_{1}$. Then do the same, starting from a point that was not covered yet by $g_{1}$. This procedure leads to the construction of $K$ smooth functions $g_{k}, K \leq Q$. By [6, page 164], $g_{k}$ are $W^{1,2}$ for a.e. $r$, since it is the restriction of a $W^{1,2}$ function to a line.

(ii) $\partial D_{r} \cap \mathcal{F} \neq \varnothing$ : in this case the set $\partial D_{r}-\mathcal{F}$, being open in $\partial D_{r}$, must be an at most countable union of open intervals $\cup_{i}\left(a_{i}, b_{i}\right)$. Then $\partial D_{r} \cap \mathcal{F}=$ $\cup_{i}\left[b_{i}, a_{i+1}\right]$. On each $\left(a_{i}, b_{i}\right)$ we can give a coherent labelling to the $\left\{\varphi_{j}\right\}$, while on the $\left[b_{i}, a_{i+1}\right]$ all the branches agree. Then we can write the multivalued graph as a superposition of $Q$ functions $g_{i}$. Each $g_{i}$ is $W^{1,2}$ : in fact, on each $\left(a_{i}, b_{i}\right)$ we can use the result from [6] again, and therefore for a.e. $r$,

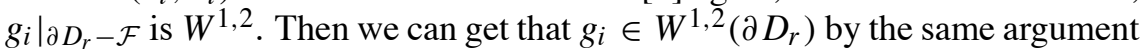
that we used to prove Theorem 5.9 by means of the Lipschitz property from Theorem 4.7, which holds on $\partial D_{r} \cap \mathcal{F}$.

Then, using Hölder's and Poincaré's inequalities, we can write (in the following computation $\lambda_{k}$ denotes the average of $g_{k}$ on $I_{k}$ and the fourth equality is justified by $\int_{I_{k}} d g_{k}=0$, which comes from the fact that $g_{k}$ takes the same value at the endpoints of $I_{k}$ ):

$$
\begin{aligned}
\left|\partial\left(C\left\llcorner Z_{r}\right)\right)(\zeta d \bar{\zeta})\right| & =|\langle C,|z|, r\rangle(\zeta \wedge d \bar{\zeta})| \\
& =\left|\sum_{j=1}^{Q}\left(\int_{\partial D_{r}-\mathcal{F}} \varphi_{j} d \overline{\varphi_{j}}+\int_{\partial D_{r} \cap \mathcal{F}} \tilde{\varphi} d \overline{\tilde{\varphi}}\right)\right| \\
& =\left|\sum_{k} \int_{I_{k}} g_{k} d \overline{g_{k}}\right|=\left|\sum_{k} \int_{I_{k}}\left(g_{k}-\lambda_{k}\right) d \overline{g_{k}}\right| \\
& \leq \sum_{k}\left(\int_{I_{k}}\left|g_{k}-\lambda_{k}\right|^{2}\right)^{\frac{1}{2}}\left(\int_{I_{k}}\left|\nabla g_{k}\right|^{2}\right)^{\frac{1}{2}} \\
& \leq \sum_{k} K n_{k} r\left(\int_{I_{k}}\left|\nabla g_{k}\right|^{2}\right) \\
& \leq K Q r \sum_{j=1}^{Q}\left(\int_{\partial D_{r}-\mathcal{F}}\left|\nabla \varphi_{j}\right|^{2}+\int_{\partial D_{r} \cap \mathcal{F}}|\nabla \tilde{\varphi}|^{2}\right) .
\end{aligned}
$$

The function $y(r)$ is weakly increasing in $r$ and absolutely continuous, being an integral; therefore it is a.e. differentiable and, thanks to (6.5) and (6.6), satisfies at a.e. $r$ (we can assume $k>1$ ) the inequality

$$
y(r) \leq k r y^{\prime}(r)+c y^{2} .
$$


By setting $v(r)=y(r)-\frac{c}{1-2 k} r^{2}$, we turn the inequality into

$$
v(r) \leq k r v^{\prime}(r)
$$

This yields

$$
v(\rho) \leq C \rho^{\frac{1}{k}}
$$

and then, adding $\frac{c}{1-2 k} r^{2}$, we get the desired estimate for $y(r)$ :

$$
y(r) \leq C r^{\delta}
$$

for some $\delta:=\frac{1}{k}>0$.

Unique continuation argument. This will conclude the proof of $\sharp_{1}$ and is inspired to the techniques used in [21], and before by Aronszajn in [2]. For this section we are going to describe our current by a multi-valued graph $D_{R} \rightarrow \mathbb{C}^{2}$, by setting the fourth (real) coordinate equal 0 . So we have a multi-valued graph $\left\{\varphi_{j}(z), \alpha_{j}(z)\right\}_{j=1}^{Q}$, with $\alpha$ purely real. The average $\tilde{\varphi}(z)$, is a $W^{1,2}$, Hölder (and bounded) function, $\tilde{\alpha}(z)$ is Lipschitz.

Lemma 6.4. There exists a constant $K$ such that, if $R$ is small enough, there exists $a W^{1,2}$ and $C^{1, \delta}$ solution $w(z): D_{R} \rightarrow \mathbb{C}$ to the equation

$$
\bar{\partial} w+v(\tilde{\varphi}, \tilde{\alpha}) \partial w=0
$$

that is a perturbation of the identity, precisely it satisfies

$$
|w(z)-z| \leq K R|z|
$$

Proof of Lemma 6.4. For a function $u$ defined on the whole of $\mathbb{C}$, we seek $w$ of the form $w=\chi_{R}(1+u(z)) z$, where $\chi_{R}$ is a radial, smooth cut-off function equal to 1 on $D_{R}$ and 0 on the complement of $D_{2 R}$. The requests on $w$ can be translated as follows

$$
\begin{gathered}
\bar{\partial} u+\chi_{R} v(\tilde{\varphi}, \tilde{\alpha}) \partial u+\chi_{R} \frac{v(\tilde{\varphi}, \tilde{\alpha})}{z}(1+u)=0, \\
|u| \leq K R .
\end{gathered}
$$

It is very important at this stage to observe that $\frac{v(\tilde{\varphi}, \tilde{\alpha})}{z}$ is an $L^{\infty}$ function thanks to the Lipschitz estimate of Corollary 5.8, although it need not be continuous; so there is some constant $K$ (independent of $R$ ) such that $\frac{v(\tilde{\varphi}, \tilde{\alpha})}{z} \leq K$ (still from Corollary 5.8 we actually know that this constant goes to 0 as $R$ goes to 0 ). The solution $u$ will be found by a fixed point method. 
Consider the ${ }^{19}$ space $H=\left\{f \in W^{1,2}(\mathbb{C})\right.$ such that $\left.\nabla f \in L^{2, \lambda}\right\}$, for some $\lambda>0$ to be chosen later. By a result due to Morrey, these functions are $\frac{\lambda}{2}$-Hölder; they also decay at infinity, therefore they are bounded. $H$ is a Banach space with the norm whose square is

$$
\begin{aligned}
\|f\|_{H}^{2} & =\|f\|_{L^{\infty}}^{2}+\|\nabla f\|_{L^{2}}^{2}+\|\nabla f\|_{L^{2, \lambda}}^{2} \\
& =\sup _{\mathbb{C}}|f|^{2}+\int_{\mathbb{C}}|\nabla f|^{2}+\sup _{x_{0} \in \mathbb{C}, \rho>0} \frac{1}{\rho^{\lambda}} \int_{B_{\rho}\left(x_{0}\right)}|\nabla f|^{2} .
\end{aligned}
$$

Define the functional $\mathcal{P}$ on $H$ that sends $f$ to $\mathcal{P}(f)$

$$
\mathcal{P}(f)(z)=\frac{1}{2 \pi i} \int_{\mathbb{C}} \frac{\chi_{R} v(\tilde{\varphi}, \tilde{\alpha}) \partial f+\chi_{R} \frac{v(\tilde{\varphi}, \tilde{\alpha})}{\xi}(1+f)}{\xi-z} d \xi d \bar{\xi}
$$

(all the functions in the integral are functions of $\xi$ ). For any fixed $z$, the integral is finite: this can be seen as follows, by breaking it up as a series of integrals over annuli $A_{n}(z)$ centered at $z$ with outer and inner radii respectively $\frac{R}{2^{n}}$ and $\frac{R}{2^{n+2}}$ (all the constants we are calling $K$ are independent of $R$ );

$$
\begin{aligned}
& \sum_{n} \frac{2^{n+2}}{R} \int_{A_{n}(z)}\left|\chi_{R} v(\tilde{\varphi}, \tilde{\alpha}) \partial f\right|+\left|\chi_{R} \frac{v(\tilde{\varphi}, \tilde{\alpha})}{\xi}\right|+\left|\chi_{R} \frac{\nu(\tilde{\varphi}, \tilde{\alpha})}{\xi} f\right| \\
& \leq K R \sum_{n} \frac{2^{n}}{R}\left(\int_{A_{n}(z)}\left|\chi_{R}\right|\right)^{\frac{1}{2}}\left(\int_{A_{n}(z)}|\partial f|^{2}\right)^{\frac{1}{2}} \\
& \quad+\sum_{n} \frac{K R}{2^{n}}+\sum_{n} \frac{K R\|f\|_{L^{\infty}}}{2^{n}},
\end{aligned}
$$

where we used $\left\|\frac{v(\tilde{\varphi}, \tilde{\alpha})}{z}\right\|_{L^{\infty}\left(D_{R}\right)} \leq K$; thanks to the finiteness of $\|\nabla f\|_{L^{2, \lambda}}^{2}$ we can bound the first term in the following way:

$$
\begin{aligned}
\sum_{n} 2^{n}\left(\int_{A_{n}(z)}\left|\chi_{R}\right|\right)^{\frac{1}{2}}\left(\int_{A_{n}(z)}|\partial f|^{2}\right)^{\frac{1}{2}} & \leq R \sum_{n}\left(\int_{A_{n}(z)}|\partial f|^{2}\right)^{\frac{1}{2}} \\
& =R \sum_{n} \frac{R^{\lambda / 2}}{2^{\frac{n \lambda}{2}}}\left(\left(\frac{2^{n}}{R}\right)^{\lambda} \int_{A_{n}(z)}|\partial f|^{2}\right)^{\frac{1}{2}} \\
& \leq K R\|\nabla f\|_{L^{2, \lambda} .}
\end{aligned}
$$

${ }^{19}$ Here we make use of the Morrey space

$$
L^{2, \lambda}:=\left\{g: \mathbb{C} \rightarrow \mathbb{R}:\|g\|_{L^{2, \lambda}}^{2}:=\sup _{x_{0} \in \mathbb{C}, \rho>0} \frac{1}{\rho^{\lambda}} \int_{B_{\rho}\left(x_{0}\right)}|g|^{2}<\infty\right\} .
$$


Note that

$$
|\mathcal{P}(0)| \leq K R
$$

and from the computations in (6.8) and (6.9) we also see that

$$
\|\mathcal{P}(f)-\mathcal{P}(0)\|_{L^{\infty}} \leq K R\|f\|_{H} .
$$

Also observe that, since we only need to integrate on $\xi \in B_{2 R}(0)$, for $|z| \geq 4 R$ we have $|\xi-z| \geq \frac{|z|}{2}$, so $|\mathcal{P}(f)|$ is bounded by $\frac{K R^{2}}{|z|}\left(\|\nabla f\|_{L^{2}}+\|f\|_{L^{\infty}}\right)$.

$\mathcal{P}(f)$ is in $W^{1,2}$ (we will shortly show that $\mathcal{P}(f) \in H$ ) and solves

$$
\bar{\partial}(\mathcal{P}(f))=-\chi_{R} v(\tilde{\varphi}, \tilde{\alpha}) \partial f-\chi_{R} \frac{v(\tilde{\varphi}, \tilde{\alpha})}{z}(1+f),
$$

since $\frac{1}{z-\xi}$ is the fundamental solution for the operator $\bar{\partial}$; in fact, $\frac{1}{z-\xi}=\frac{\partial}{\partial z}(\ln \mid z-$ $\xi \mid)$, and $\bar{\partial} \partial=i \Delta$, compare [9, page 17].

Therefore, what we are looking for is a fixed point for $\mathcal{P}$ in $H$. Observe that $\mathcal{P}$ is an affine functional, therefore, to show that it is a contraction in $H$, it will be enough to show

$$
\begin{gathered}
\mathcal{P}(0) \in H, \\
\|\mathcal{P}(f)-\mathcal{P}(0)\|_{H} \leq k\|f\|_{H},
\end{gathered}
$$

for any $f$ and for some $0<k<1$. From (6.11),

$$
\|\bar{\partial}(\mathcal{P}(f)-\mathcal{P}(0))\|_{L^{2}} \leq K R\left(\|\nabla f\|_{L^{2}}+\|f\|_{L^{\infty}}\right)
$$

and, since $\mathcal{P}(f)-\mathcal{P}(0)$ decays at infinity as $\frac{1}{|z|}$, we can integrate by parts to get

$$
\begin{aligned}
\|\nabla(\mathcal{P}(f)-\mathcal{P}(0))\|_{L^{2}} & =K\|\bar{\partial}(\mathcal{P}(f)-\mathcal{P}(0))\|_{L^{2}} \\
& \leq K R\left(\|\nabla f\|_{L^{2}}+\|f\|_{L^{\infty}}\right) .
\end{aligned}
$$

The fact that

$$
\|D(\mathcal{P}(f)-\mathcal{P}(0))\|_{L^{2, \lambda}} \leq K R\left(\|D f\|_{L^{2}}+\|f\|_{L^{2, \lambda}}\right)
$$

follows from equation (6.11) by [15, Theorem 5.4.1, page 146].

The last estimate, together with (6.10) and (6.12), implies

$$
\|\mathcal{P}(f)-\mathcal{P}(0)\|_{H} \leq K R\|f\|_{H} .
$$

Similarly we can show that $\mathcal{P}(0) \in H$, with $\|\mathcal{P}(0)\|_{H} \leq K R$. If $R$ is small enough (recall that $\frac{v}{z} \leq K$ independently of $R$ ), we have a contraction and by Caccioppoli's fixed point theorem we have the existence of a unique fixed point $u$ for $\mathcal{P}$ and

$$
\|u\|_{L^{\infty}} \leq 2 K R .
$$

So we have a Hölder function $w=z(1+u)$ solution to $(6.7)$. Since $v(\tilde{\varphi}, \tilde{\alpha})$ is Hölder-continuous of exponent $\delta$ thanks to Theorem 6.1, by means of a Shaudertype estimate $w$ is $C^{1, \delta}$. 
Remark 6.5. Observe that $|w(z)-z| \leq K R|z|$ implies that at $0, \partial w \approx 1, \bar{\partial} w \approx 0$, with perturbations of order $K R$. By taking $R$ smaller if necessary, we can assume, since $w$ is $C^{1, \delta}$, that $\partial w$ and $\bar{\partial} w$ stay as close as we like to 1 and 0 in $B_{R}$.

Core of the proof of Theorem 5.1. We are now ready to complete the proof of non-accumulation, which will go on until the end of this section. Take the function $G: \mathbb{C}^{3} \rightarrow \mathbb{C}^{3}$ given by

$$
G(z, \zeta, a)=(z, \zeta-\tilde{\varphi}(z), a-\tilde{\alpha}(z))
$$

and consider the pushforward $\Gamma:=G_{*} C$. The map $G$ is proper (if $K$ is compact, $G^{-1}(K)$ is closed by continuity and bounded since the average function is $L^{\infty}$ ) and $W^{1,2}$ : this gives that the pushforward is well-defined and commutes with the boundary operator. The point here is that the $W^{1,2}$ function $G$, from a domain in $\mathbb{R}^{2}$ into $\mathbb{R}^{3}$, can be approximated by $C^{1}$ functions $G_{\varepsilon}$ as $\varepsilon \rightarrow 0$ so that the minors $D G_{\varepsilon}$ converge weakly in $L^{1}$ to the minors of $D G$ (see [8, Propositions 2 and 3, pages 232-233]). This implies that $\partial \Gamma=0$ and that the current $\Gamma$ is described by the multi-valued graph

$$
\left\{\sigma_{j}, \tau_{j}\right\}=\left\{\varphi_{j}-\tilde{\varphi}, \alpha_{j}-\tilde{\alpha}\right\}
$$

From (5.15), the smooth parts of $\left\{\sigma_{j}\right\}$ solve

$$
\bar{\partial} \sigma_{j}+v(\tilde{\varphi}, \tilde{\alpha}) \partial \sigma_{j}+\sum_{k=1}^{Q} S_{j}^{k} \sigma_{k}+\sum_{k=1}^{Q} T_{j}^{k} \tau_{k}=0
$$

with $\left|T_{j}^{k}\right|,\left|S_{j}^{k}\right| \leq K\left(1+\sum_{i=1}^{Q}\left|\nabla \varphi_{i}\right|+\sum_{i=1}^{Q}\left|\nabla \alpha_{i}\right|\right)$. Therefore, by the Hölder estimate in Theorem 6.1, $\left|T_{j}^{k}\right|,\left|S_{j}^{k}\right|$ are in $L^{2}\left(D_{R}\right)$. As for $\left\{\tau_{j}\right\}$, from (5.10) and (5.11) we have that

$$
\nabla \alpha_{j}(z)=h\left(z, \varphi_{j}(z), \alpha_{j}(z)\right),
$$

for a smooth $\mathbb{R}^{2}$-valued $h$, so

$$
\bar{\partial} \tau_{j}=\sum_{k=1}^{Q} A_{j}^{k} \sigma_{k}+\sum_{k=1}^{Q} B_{j}^{k} \tau_{k}
$$

with $A_{j}^{k}, B_{j}^{k}$ bounded; for $\partial \tau_{j}$ we have a similar equation, since the $\tau_{j}$ are real (so the equation we wrote actually contains the whole information on the two real derivatives). Putting them together (we keep writing $A, B$ although these coefficient are different)

$$
\bar{\partial} \tau_{j}+v(\tilde{\varphi}, \tilde{\alpha}) \partial \tau_{j}+\sum_{k=1}^{Q} A_{j}^{k} \sigma_{k}+\sum_{k=1}^{Q} B_{j}^{k} \tau_{k}=0
$$

with $A_{j}^{k}, B_{j}^{k}$ bounded. 


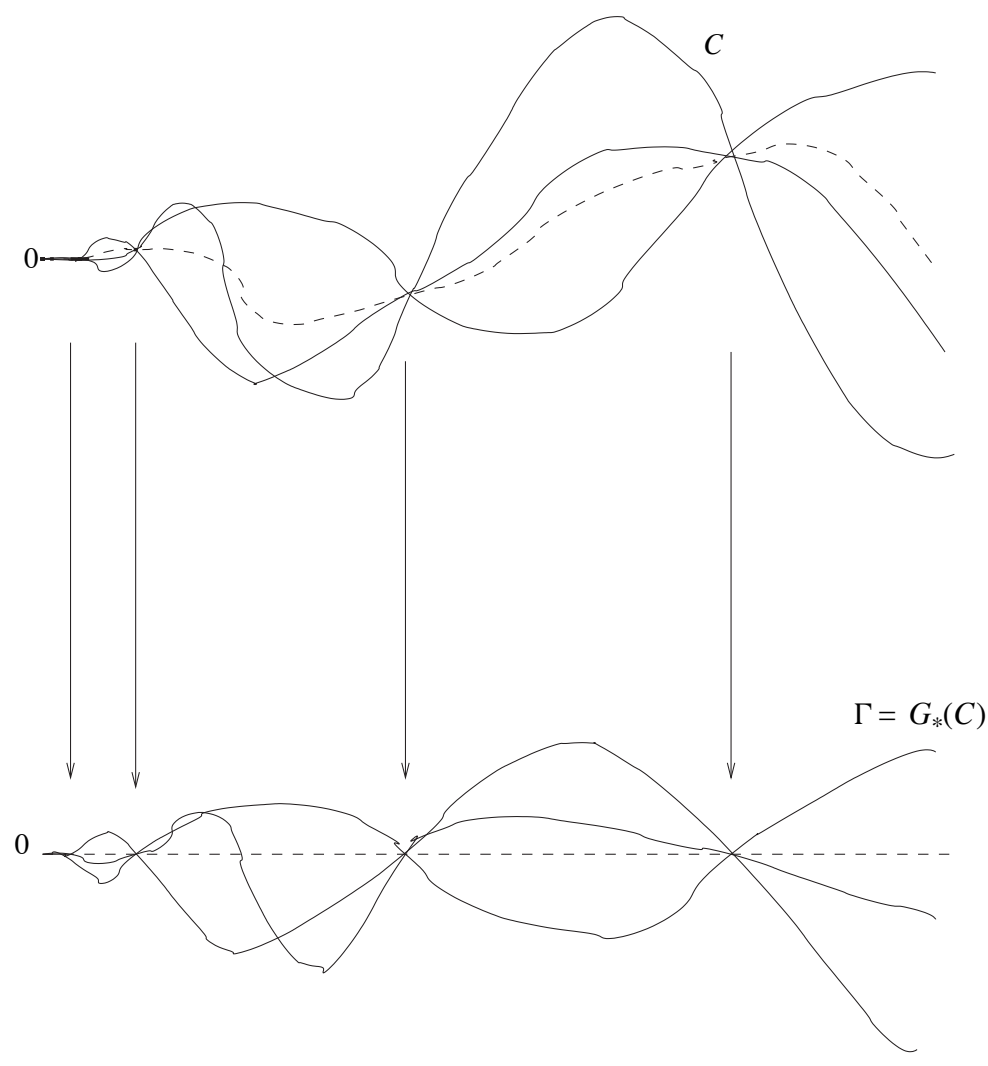

Figure 6.1. A sketch for a 3-valued graph. The average is the dotted line in the first picture. By subtracting it, we get a new 3-valued graph: the points of multiplicity 3 are turned into zeros. The new 3-valued graph still represents a boundaryless current, thanks to the $W^{1,2}$-estimate on the average.

Observe that singularities of order $Q$ in $C$ have the property that all the branches coincide at those points, therefore they are zeros of the multi-valued graph $\left\{\sigma_{j}, \tau_{j}\right\}$.

Assume by contradiction the existence of a sequence of singular points in Sing $Q$ accumulating onto 0 .

Then we can take $N$ points $q_{n} \in \mathcal{F}=\pi\left(\operatorname{Sing}{ }^{Q}\right)$ which lie in $D_{r}$, with $N$ as large as we want and $r<R$ arbitrarily small and $\left\{\sigma_{j}, \tau_{j}\right\}\left(q_{n}\right)=0$, for $n=$ $1, \ldots, N$. In the estimates to come, one should always pay attention to the fact that the constants obtained must not depend on the chosen $N$ and $r$, unless otherwise specified.

Define the function

$$
g(z):=\Pi_{i=1}^{N}\left(w(z)-w\left(q_{i}\right)\right)
$$


with the $w$ obtained in the previous lemma. Then $g$ is a $C^{1}, W^{1,2}$ function and it solves on $D_{R}$

$$
\bar{\partial} g+v(\tilde{\varphi}, \tilde{\alpha}) \partial g=0 .
$$

Take $F: \mathbb{C}^{3} \rightarrow \mathbb{C}^{3}$

$$
F(z, \zeta, a)=\left(z, \chi_{r}(z) \frac{\zeta}{g(z)}, \chi_{r}(z) \frac{a}{g(z)}\right),
$$

where $\chi_{r}$ is a radial, smooth cut-off, 1 on $B_{r}, 0$ on the complement of $B_{2 r}$, with gradient bounded by $\frac{K}{r}$; we are going to analyse the pushforward $F_{*}\left(G_{*}(C)\right)$. First observe that, on any set of the form $D_{R} \backslash \cup_{i=1}^{N} B_{\delta}\left(q_{i}\right)$ for $\delta$ as small as we want, $F$ is a $C^{1}$, Lipschitz and proper function. Set

$$
A_{\delta}:=\left(D_{R} \backslash \cup_{i=1}^{N} B_{\delta}\left(q_{i}\right)\right) \times \mathbb{C} \times \mathbb{C} ;
$$

we can restrict $\Gamma$ to $A_{\delta}$, writing $\Gamma_{\delta}:=\Gamma\left\llcorner A_{\delta}\right.$, and then the pushforward $\Delta_{\delta}:=$ $F_{*}\left(\Gamma_{\delta}\right)$ is a well defined i.m. rectifiable current with finite mass, and it can develop boundary only on $\left(\cup_{i=1}^{N} \partial B_{\delta}\left(q_{i}\right)\right) \times \mathbb{C} \times \mathbb{C}$. Now we will prove

Lemma 6.6. Sending $\delta \rightarrow 0$, we can define the pushforward $\Delta:=F_{*}(\Gamma)=$ $F_{*}\left(G_{*}(C)\right)$ on the whole of $D_{R} \times \mathbb{C} \times \mathbb{C}$, and $\Delta$ is a boundaryless current of finite mass. Then we can rewrite the following relation

$$
\Delta(d \zeta d \bar{\zeta})=\partial \Delta(\zeta d \bar{\zeta})
$$

as a standard integration by parts formula, where both integrals are finite:

$$
\int_{B_{2 r}(0)} \sum_{j}\left|\bar{\partial}\left(\frac{\chi_{r} \sigma_{j}}{g}\right)\right|^{2}=\int_{B_{2 r}(0)} \sum_{j}\left|\partial\left(\frac{\chi_{r} \sigma_{j}}{g}\right)\right|^{2} .
$$

Remark 6.7. Formula (6.17) is the only thing we will need in the sequel. The finiteness of the integrals was not clear in the analogous formula used in [21].

Remark 6.8. In this formula $\nabla\left(\frac{\chi_{r} \sigma_{j}}{g}\right)$ is understood to be 0 on the set $\mathcal{F}=\pi\left(\operatorname{Sing}^{Q}\right)$. The reason for this will be clear during the proof. On the complement $D_{R} \backslash$ $\pi\left(\operatorname{Sing}^{Q}\right)$ the gradient is well-defined since the functions are smooth except at the isolated points $\pi\left(\operatorname{Sing}^{\leq} Q^{-1}\right)$.

Proof of Lemma 6.6. From what we said before, $\Delta$ can develop boundary only on $\left(\cup_{i=1}^{N} q_{i}\right) \times \mathbb{C} \times \mathbb{C}$. Moreover, $\Delta$ is described by the multi-valued graph

$$
\left\{\frac{\chi_{r} \sigma_{j}}{g}, \frac{\chi_{r} \tau_{j}}{g}\right\}_{j=1}^{Q}
$$


From Theorem 4.7, this multi-valued graph is bounded on $D_{R}$, indeed we only have to check it at the points $q_{i}$ : on some neighbourhood of a chosen $q_{k}$, thanks to Corollary 5.8,

$$
\left|\sigma_{j}(z)\right|=\left|\sigma_{j}(z)-\sigma\left(q_{k}\right)\right| \leq K\left|z-q_{k}\right|
$$

By Lagrange's theorem, if the mentioned neighbourhood was chosen small enough (its size should be much smaller than the distances between the $q_{i}$ 's), then $g(z) \approx$ $\Pi_{i=1}^{N}\left(z-q_{i}\right)$; more precisely, $K_{1} \Pi_{i=1}^{N}\left|z-q_{i}\right| \leq|g(z)| \leq K_{2} \Pi_{i=1}^{N}\left|z-q_{i}\right|$ with $K_{1}, K_{2}$ close to 1 (the perturbation is due to the perturbations $\partial w \approx 1$ and $\bar{\partial} w \approx 0$ ). Therefore

$$
\left|\sigma_{j}(z)\right| \leq K_{\left\{q_{i}\right\}}|g(z)|,
$$

where $K_{\left\{q_{i}\right\}}$ is a constant that depends on the choices of $r$ and the set $\left\{q_{i}\right\}$ (more precisely the constant is of order $\Pi_{i \neq j}\left|q_{i}-q_{j}\right|^{-1}$ ). This will not be problematic, all that matters to us is the fact that

$$
\left\{\frac{\chi_{r} \sigma_{j}}{g}, \frac{\chi_{r} \tau_{j}}{g}\right\}_{j=1}^{Q}
$$

is bounded. We further observe that, thanks to the equation solved by $g$, the multivalued graph

$$
\left\{\frac{\sigma_{j}}{g}, \frac{\tau_{j}}{g}\right\}_{j=1}^{Q}
$$

satisfies, on $D_{R} \backslash \mathcal{F}$,

$$
\begin{aligned}
& \bar{\partial}\left(\frac{\sigma_{j}}{g}\right)+v(\tilde{\varphi}, \tilde{\alpha}) \partial\left(\frac{\sigma_{j}}{g}\right)+\sum_{k=1}^{Q} S_{j}^{k}\left(\frac{\sigma_{k}}{g}\right)+\sum_{k=1}^{Q} T_{j}^{k}\left(\frac{\tau_{k}}{g}\right)=0, \\
& \bar{\partial}\left(\frac{\tau_{j}}{g}\right)+v(\tilde{\varphi}, \tilde{\alpha}) \partial\left(\frac{\tau_{j}}{g}\right)+\sum_{k=1}^{Q} A_{j}^{k}\left(\frac{\sigma_{k}}{g}\right)+\sum_{k=1}^{Q} B_{j}^{k}\left(\frac{\tau_{k}}{g}\right)=0,
\end{aligned}
$$

with the coefficients $A, B, S, T$ as above.

Step 1: $\Delta$ has finite mass. Remark that the closed set $\mathcal{F}=\pi\left(\operatorname{Sing}{ }^{Q}\right)$ is included in $\left\{z: \forall i \sigma_{i}(z)=\tau_{i}(z)=0\right\}$. The integer multiplicity rectifiable current $\Delta_{\delta}$ possesses a.e. on $\mathcal{F} \backslash \cup_{i=1}^{N} B_{\delta}\left(q_{i}\right)$ an approximate tangent plane that must be horizontal, i.e. it must be the plane $(z, 0,0)$. Indeed, this is true at any point of density 1 of the set $\left\{z: \forall i \sigma_{i}(z)=\tau_{i}(z)=0\right\} \backslash \cup_{i=1}^{N} B_{\delta}\left(q_{i}\right)$, as can be seen from the definition of tangent plane (see [8] page 92).

Let us observe the action of $\Delta_{\delta}$ on $d \zeta \wedge d \bar{\zeta}$. By the observation we just made, this action gives 0 on $\mathcal{F}$, therefore we can extend $\nabla\left(\frac{\chi_{r} \sigma_{j}}{g}\right)$ to be 0 on $\mathcal{F} \backslash \cup_{i=1}^{N} B_{\delta}\left(q_{i}\right)$ 
(compare Remark 6.8). With this understood we can write:

$$
\begin{aligned}
\Delta_{\delta}(d \zeta \wedge d \bar{\zeta}) & =\int_{B_{2 r} \backslash \cup_{i=1}^{N} B_{\delta}\left(q_{i}\right)} \sum_{j} d\left(\frac{\chi_{r} \sigma_{j}}{g}\right) \wedge d \overline{\left(\frac{\chi_{r} \sigma_{j}}{g}\right)} \\
& =\int_{B_{2 r} \backslash \cup_{i=1}^{N} B_{\delta}\left(q_{i}\right)} \sum_{j}\left|\bar{\partial}\left(\frac{\chi_{r} \sigma_{j}}{g}\right)\right|^{2}-\left|\partial\left(\frac{\chi_{r} \sigma_{j}}{g}\right)\right|^{2} .
\end{aligned}
$$

By (6.19) and the triangle inequality $|a-b|^{2} \geq \frac{|b|^{2}}{2}-|a|^{2}$ we get, recalling that $|\nu| \leq$ $\varepsilon$, (the integrals in the following lines are performed on an arbitrary measurable set disjoint from $\left.\cup_{i=1}^{N} B_{\delta}\left(q_{i}\right)\right)$ :

$$
\begin{aligned}
\int \sum_{j}\left|\sum_{k=1}^{Q} S_{j}^{k}\left(\frac{\sigma_{k}}{g}\right)+\sum_{k=1}^{Q} T_{j}^{k}\left(\frac{\tau_{k}}{g}\right)\right|^{2} \\
=\int \sum_{j}\left|\bar{\partial}\left(\frac{\sigma_{j}}{g}\right)+v(\tilde{\varphi}, \tilde{\alpha}) \partial\left(\frac{\sigma_{j}}{g}\right)\right|^{2} \\
\geq \int \sum_{j}\left(\frac{\left|\bar{\partial}\left(\frac{\sigma_{j}}{g}\right)\right|^{2}}{2}-\varepsilon^{2}\left|\partial\left(\frac{\sigma_{j}}{g}\right)\right|^{2}\right) \\
=\int \sum_{j}\left(\frac{1}{2}+\varepsilon^{2}\right)\left|\bar{\partial}\left(\frac{\sigma_{j}}{g}\right)\right|^{2} \\
\quad+\varepsilon^{2} \sum_{j}\left(\left|\bar{\partial}\left(\frac{\chi_{r} \sigma_{j}}{g}\right)\right|^{2}-\left|\partial\left(\frac{\chi_{r} \sigma_{j}}{g}\right)\right|^{2}\right) \\
=\int \sum_{j}\left(\frac{1}{2}+\varepsilon^{2}\right)\left|\bar{\partial}\left(\frac{\sigma_{j}}{g}\right)\right|^{2}+\varepsilon^{2} \Delta_{\delta}(d \zeta \wedge d \bar{\zeta}) \\
=\int \sum_{j}\left(\frac{1}{2}+\varepsilon^{2}\right)\left|\bar{\partial}\left(\frac{\sigma_{j}}{g}\right)\right|^{2}+\varepsilon^{2} \partial \Delta_{\delta}(\zeta \wedge d \bar{\zeta}) .
\end{aligned}
$$

Notice that the first term at the beginning of the last chain of inequalities is finite, from the condition on the $T$ 's and $S$ 's, and the fact that $\frac{\sigma_{k}}{g}, \frac{\tau_{k}}{g}$ is bounded.

Let us restrict to a small ball $B_{\lambda}\left(q_{i}\right)$ : we will show that

$$
\lim _{\rho \rightarrow 0} \int_{B_{\lambda}\left(q_{i}\right) \backslash B_{\rho}\left(q_{i}\right)} \sum_{j}\left|\bar{\partial}\left(\frac{\sigma_{j}}{g}\right)\right|^{2}
$$

is finite; the global finiteness on $D_{R}$ will follow since the $q_{i}$ 's are finite and there are no poles elsewhere. In a first moment we are going to construct a sequence $\rho_{n} \downarrow 0$ for which $M\left(\partial \Delta_{\rho_{n}}\right)$ is equibounded. 
Since $\Delta_{\rho}=F_{*}\left(\Gamma_{\rho}\right)$ and $\|\nabla F\|_{L^{\infty}\left(B_{\lambda}\left(q_{i}\right) \backslash B_{\rho}\left(q_{i}\right)\right)} \leq \frac{K}{\rho}$, from [8, page 134], we get

$$
M\left(\partial \Delta_{\rho}\right) \leq \frac{K}{\rho} M\left(\partial \Gamma_{\rho}\right) .
$$

Moreover, from slicing theory, see [8, Proposition 2, page 154],

$$
\begin{aligned}
\frac{1}{(\lambda / n)^{2}} \int_{0}^{\frac{\lambda}{n}} M\left(\partial \Gamma_{\rho}\right) d \rho & =\frac{1}{(\lambda / n)^{2}} \int_{0}^{\frac{\lambda}{n}} M(\langle\Gamma,|z|, \rho\rangle) \\
& \leq \frac{1}{(\lambda / n)^{2}} M\left(\Gamma\left\llcorner\left(B_{\frac{\lambda}{n}}\left(q_{i}\right) \times \mathbb{C}^{2}\right)\right)\right.
\end{aligned}
$$

and this is bounded as $n \rightarrow \infty$ by the monotonicity formula, since the tangent is horizontal at $\left(q_{i}, 0,0\right)$ and the multiplicity of this point is $Q$. Then

$$
\frac{1}{(\lambda / n)} \int_{0}^{\frac{\lambda}{n}} M\left(\partial \Gamma_{\rho}\right) d \rho \leq K \frac{\lambda}{n}
$$

so by the mean-value theorem there is $\frac{\lambda}{4 n} \leq \rho_{n} \leq \frac{\lambda}{n}$ such that

$$
M\left(\partial \Gamma_{\rho_{n}}\right) \leq 2 \frac{1}{(\lambda / n)} \int_{0}^{\frac{\lambda}{n}} M\left(\partial \Gamma_{\rho}\right) d \rho \leq 2 K \frac{\lambda}{n} \leq 8 K \rho_{n} .
$$

Now (6.23) yields that $M\left(\partial \Delta_{\rho_{n}}\right)$ are equibounded.

As observed above, $\frac{\sigma_{j}}{g}$ is $L^{\infty}$, therefore the function $\zeta$ is bounded on $\Delta$, so there is some constant which bounds uniformly in $n$

$$
\left|\partial \Delta_{\rho_{n}}(\zeta d \bar{\zeta})\right|
$$

This yields, together with the inequality (6.22) used with $\delta=\rho_{n}$ on the set $B_{\lambda}\left(q_{i}\right) \backslash$ $B_{\rho_{n}}\left(q_{i}\right)$,

$$
\lim _{\rho_{n} \rightarrow 0} \int_{B_{\lambda}\left(q_{i}\right) \backslash B_{\rho_{n}}\left(q_{i}\right)} \sum_{j}\left|\bar{\partial}\left(\frac{\sigma_{j}}{g}\right)\right|^{2}<\infty ;
$$

consequently

$$
\lim _{\rho \rightarrow 0} \int_{B_{\lambda}\left(q_{i}\right) \backslash B_{\rho}\left(q_{i}\right)} \sum_{j}\left|\bar{\partial}\left(\frac{\sigma_{j}}{g}\right)\right|^{2}<\infty,
$$

since this integral is a monotone function of $\rho$, so the limit must exist and it is enough to check in on a sequence. Once we have the finiteness of

$$
\int_{D_{R}} \sum_{j}\left|\bar{\partial}\left(\frac{\sigma_{j}}{g}\right)\right|^{2}
$$


using $\left|\Delta_{\rho_{n}}(d \zeta \wedge d \bar{\zeta})\right|=\left|\partial \Delta_{\rho_{n}}(\zeta d \bar{\zeta})\right|<\infty$ again, by (6.21) we also get the finiteness of

$$
\int_{D_{R}} \sum_{j}\left|\partial\left(\frac{\sigma_{j}}{g}\right)\right|^{2} \text {. }
$$

This implies that the Jacobian minors of $\frac{\sigma_{j}}{g}$ are in $L^{1}$, so the finiteness of the mass can be obtained by the Area formula ${ }^{20}$, see [8, page 225].

Step 2: $\Delta$ has no boundary. As said above, we only have to exclude boundary terms localized at the points $q_{i}$. As before, we restrict ourselves to $\Delta\left\llcorner B_{\lambda}\left(q_{i}\right) \times\right.$ $\mathbb{C}^{2}$. During this step, we will keep denoting this current by $\Delta$. To simplify things, we will test $\partial \Delta$ only on the 1 -forms $\chi_{\rho}(z) \zeta d \bar{\zeta}$, which is needed for the integration by parts formula (6.17); the proof for other 1 -forms is similar ${ }^{21}$. Since the possible boundary in the interior of $D_{R}$ is localized only in $q_{i} \times \mathbb{C}^{2}$, the result will be the same for any $\rho$.

$$
\partial \Delta\left(\chi_{\rho}(z) \zeta d \bar{\zeta}\right)=\Delta\left(d \chi_{\rho} \wedge \zeta d \bar{\zeta}\right)+\Delta\left(\chi_{\rho} d \zeta \wedge d \bar{\zeta}\right)
$$

From the previous step,

$$
\left|\Delta\left(\chi_{\rho} d \zeta \wedge d \bar{\zeta}\right)\right| \leq \int_{B_{2 \rho}\left(q_{i}\right)} \sum_{j}\left|\nabla\left(\frac{\sigma_{j}}{g}\right)\right|^{2} \rightarrow 0
$$

for $\rho \rightarrow 0$. Let us now analyse the first term:

$$
\begin{aligned}
\left|\Delta\left(d \chi_{\rho} \wedge \zeta d \bar{\zeta}\right)\right| & =\left|\int_{B_{2 \rho}\left(q_{i}\right) \backslash B_{\rho}\left(q_{i}\right)} \sum_{j} \partial \chi_{\rho} \frac{\sigma_{j}}{g} \bar{\partial}\left(\frac{\sigma_{j}}{g}\right)\right| \\
& \leq \int_{B_{2 \rho}\left(q_{i}\right) \backslash B_{\rho}\left(q_{i}\right)} \frac{K}{\rho}\left\|\frac{\sigma_{j}}{g}\right\|_{L^{\infty}}\left|\nabla\left(\frac{\sigma_{j}}{g}\right)\right|
\end{aligned}
$$

and by Hölder's inequality

$$
\leq\left\|\frac{\sigma_{j}}{g}\right\|_{L^{\infty}} \frac{K}{\rho} 2 \rho\left(\int_{B_{2 \rho}\left(q_{i}\right)} \sum_{j}\left|\nabla\left(\frac{\sigma_{j}}{g}\right)\right|^{2}\right)^{\frac{1}{2}} .
$$

This integral goes to 0 as $\rho \rightarrow 0$ thanks to the previous step. So there is no boundary term at any of the $q_{i}$ when we test on the one form $\zeta d \bar{\zeta}$.

${ }^{20}$ Recall that it is enough to apply the Area formula to the smooth parts of the current $\Delta$ that are above $D_{R} \backslash \mathcal{F}$. The rest of the current lies in $\mathcal{F}$, which has finite measure.

${ }^{21}$ For the reader who is familiar with the support theorem for Flat-currents (see [8, page 525]), we remark that the absence of boundary can be obtained by showing, via an approximation argument, that $\partial \Delta$ is a Flat 1 -current. The quoted theorem then implies that $\partial \Delta=0$. 
We are now ready to finish the proof of non accumulation started before Lemma 6.6: recall that we assumed, by contradiction, the existence of $N$ points $q_{n} \in$ $\mathcal{F}=\pi\left(\operatorname{Sing}^{Q}\right)$ which lie in $D_{r}$, with $N$ as large as we want and $r<R$ arbitrarily small and $\left\{\sigma_{j}, \tau_{j}\right\}\left(q_{n}\right)=0$, for $n=1, \ldots, N$. From Leibnitz rule and (6.19)

$$
\begin{aligned}
\int_{B_{2 r}} \sum_{j}\left|\bar{\partial}\left(\frac{\chi_{r} \sigma_{j}}{g}\right)\right|^{2} \leq K r^{-2} \int_{B_{2 r} \backslash B_{r}} \sum_{j}\left|\frac{\sigma_{j}}{g}\right|^{2}+\int_{B_{2 r}} \sum_{j}\left|\chi_{r}\right|^{2}\left|\bar{\partial}\left(\frac{\sigma_{j}}{g}\right)\right|^{2} \\
=K r^{-2} \int_{B_{2 r} \backslash B_{r}} \sum_{j}\left|\frac{\sigma_{j}}{g}\right|^{2}+\int_{B_{2 r}} \sum_{j}|v(\tilde{\varphi}, \tilde{\alpha})|^{2}\left|\chi_{r}\right|^{2}\left|\partial\left(\frac{\sigma_{j}}{g}\right)\right|^{2} \\
+K \int_{B_{2 r}}\left(1+\sum_{i=1}^{Q}\left|\nabla \varphi_{i}\right|^{2}+\sum_{i=1}^{Q}\left|\nabla \alpha_{i}\right|^{2}\right) \sum_{j}\left(\left|\chi_{r} \frac{\sigma_{j}}{g}\right|^{2}+\left|\chi_{r} \frac{\tau_{j}}{g}\right|^{2}\right) .
\end{aligned}
$$

Now, using $|\nu| \leq \varepsilon$ and (6.17) (notice that the previous lemma and the fact that $\left\{\frac{\sigma}{g}, \frac{\tau}{g}\right\}$ is bounded guarantee the finiteness of all terms),

$$
\begin{aligned}
\int_{B_{2 r}} \sum_{j}|v(\tilde{\varphi}, \tilde{\alpha})|^{2}\left|\chi_{r} \partial\left(\frac{\sigma_{j}}{g}\right)\right|^{2} & =\int_{B_{2 r}} \sum_{j}|v(\tilde{\varphi}, \tilde{\alpha})|^{2}\left|-\partial \chi_{r}\left(\frac{\sigma_{j}}{g}\right)+\partial\left(\frac{\chi_{r} \sigma_{j}}{g}\right)\right|^{2} \\
& \leq K \varepsilon^{2} r^{-2} \int_{B_{2 r} \backslash B_{r}} \sum_{j}\left|\frac{\sigma_{j}}{g}\right|^{2}+\varepsilon^{2} \int_{B_{2 r}}\left|\bar{\partial}\left(\frac{\chi_{r} \sigma_{j}}{g}\right)\right|^{2} .
\end{aligned}
$$

Putting all together, with a further use of (6.17) on the l.h.s., we get

$$
\begin{aligned}
\int_{B_{2 r}} \sum_{j}\left|\nabla\left(\frac{\chi_{r} \sigma_{j}}{g}\right)\right|^{2}=2 \int_{B_{2 r}} \sum_{j}\left|\bar{\partial}\left(\frac{\chi_{r} \sigma_{j}}{g}\right)\right|^{2} \\
\leq K r^{-2} \int_{B_{2 r}-B_{r}} \sum_{j}\left|\frac{\sigma_{j}}{g}\right|^{2} \\
\quad+K \int_{B_{2 r}}\left(1+\sum_{i=1}^{Q}\left|\nabla \varphi_{i}\right|^{2}+\sum_{i=1}^{Q}\left|\nabla \alpha_{i}\right|^{2}\right) \sum_{j}\left(\left|\chi_{r} \frac{\sigma_{j}}{g}\right|^{2}+\left|\chi_{r} \frac{\tau_{j}}{g}\right|^{2}\right) .
\end{aligned}
$$

Similarly, from (6.20), and using the analogous partial integration

$$
\int_{B_{2 r}(0)} \sum_{j}\left|\bar{\partial}\left(\frac{\chi_{r} \tau_{j}}{g}\right)\right|^{2}=\int_{B_{2 r}(0)} \sum_{j}\left|\partial\left(\frac{\chi_{r} \tau_{j}}{g}\right)\right|^{2},
$$

we get

$$
\begin{aligned}
& \int_{B_{2 r}} \sum_{j}\left|\nabla\left(\frac{\chi_{r} \tau_{j}}{g}\right)\right|^{2} \leq K r^{-2} \int_{B_{2 r} \backslash B_{r}} \sum_{j}\left|\frac{\tau_{j}}{g}\right|^{2} \\
& \quad+K \int_{B_{2 r}}\left(1+\sum_{i=1}^{Q}\left|\nabla \varphi_{i}\right|^{2}+\sum_{i=1}^{Q}\left|\nabla \alpha_{i}\right|^{2}\right) \sum_{j}\left(\left|\chi_{r} \frac{\sigma_{j}}{g}\right|^{2}+\left|\chi_{r} \frac{\tau_{j}}{g}\right|^{2}\right) .
\end{aligned}
$$


Set now $v:=\max _{j}\left\{\left|\chi_{r} \frac{\sigma_{j}}{g}\right|,\left|\chi_{r} \frac{\tau_{j}}{g}\right|\right\}$. This function is $W^{1,2}$ : indeed, this is true on $D_{2 r} \backslash \pi\left(\right.$ Sing $\left.^{\leq} Q\right)$, since it is the maximum of $W^{1,2}$ functions; then by arguments already used,

- $\pi(\operatorname{Sing} \leq Q-1)$ are isolated points so we can extend the $W^{1,2}$ estimate to $D_{2 r} \backslash$ $\pi(\operatorname{Sing} Q)$;

- then we extend to $D_{2 r} \backslash\left(\cup_{i=1}^{N} B_{\delta}\left(q_{i}\right) \cap \mathcal{F}\right)$ for any arbitrarily small $\delta$, thanks to the fact that $v=0$ on on $\operatorname{Sing} Q \backslash\left\{q_{i}\right\}$;

- finally, sending $\delta \rightarrow 0$, to the whole of $D_{R}$ since the $q_{i}$ are isolated.

Also observe that, by the Cauchy-Schwarz inequality, $|\nabla(|v|)| \leq|\nabla v|$, so

$$
\int_{B_{2 r}}|\nabla v|^{2} \leq \int_{B_{2 r}} \sum_{j}\left|\nabla\left(\frac{\chi_{r} \sigma_{j}}{g}\right)\right|^{2}+\sum_{j}\left|\nabla\left(\frac{\chi_{r} \tau_{j}}{g}\right)\right|^{2},
$$

so (6.24) and (6.25) imply

$$
\begin{aligned}
\int_{B_{2 r}}|\nabla v|^{2} \leq & K r^{-2} \int_{B_{2 r} \backslash B_{r}} \sum_{j}\left(\left|\frac{\sigma_{j}}{g}\right|^{2}+\left|\frac{\tau_{j}}{g}\right|^{2}\right) \\
& +K \int_{B_{2 r}}\left(1+\sum_{i=1}^{Q}\left|\nabla \varphi_{i}\right|^{2}+\sum_{i=1}^{Q}\left|\nabla \alpha_{i}\right|^{2}\right) v^{2} .
\end{aligned}
$$

Recall that $\left(1+\sum_{i=1}^{Q}\left|\nabla \varphi_{i}\right|^{2}+\sum_{i=1}^{Q}\left|\nabla \alpha_{i}\right|^{2}\right)$ is $L^{1}$ by Theorem 6.1 (Hölder estimate); then, by [15, Lemma 5.4.1], we get the existence of $\delta>0$ such that the last term can be bounded by

$$
\int_{B_{2 r}}\left(1+\sum_{i=1}^{Q}\left|\nabla \varphi_{i}\right|^{2}+\sum_{i=1}^{Q}\left|\nabla \alpha_{i}\right|^{2}\right) v^{2} \leq K r^{\delta} \int_{B_{2 r}}|\nabla v|^{2},
$$

so we can write

$$
r^{2} \int_{B_{2 r}}|\nabla v|^{2} \leq K \int_{B_{2 r} \backslash B_{r}} \sum_{j}\left(\left|\frac{\sigma_{j}}{g}\right|^{2}+\left|\frac{\tau_{j}}{g}\right|^{2}\right) ;
$$

now, since $v \in W_{0}^{1,2}\left(B_{2 r}\right)$, by Poincaré's inequality

$$
\int_{B_{2 r}} v^{2} \leq K \int_{B_{2 r} \backslash B_{r}} \sum_{j}\left(\left|\frac{\sigma_{j}}{g}\right|^{2}+\left|\frac{\tau_{j}}{g}\right|^{2}\right) .
$$

Since $\sum_{j}\left(\left|\chi_{r} \frac{\sigma_{j}}{g}\right|^{2}+\left|\chi_{r} \frac{\tau_{j}}{g}\right|^{2}\right) \leq 2 Q v^{2}$ by definition of $v$, and $\chi_{r}=1$ on $B_{r}$, the last inequality implies the following Carleman-type estimate

$$
\int_{B_{r / 4}} \sum_{j}\left(\left|\frac{\sigma_{j}}{g}\right|^{2}+\left|\frac{\tau_{j}}{g}\right|^{2}\right) \leq K \int_{B_{2 r} \backslash B_{r}} \sum_{j}\left(\left|\frac{\sigma_{j}}{g}\right|^{2}+\left|\frac{\tau_{j}}{g}\right|^{2}\right),
$$


with $K$ independent of $r$ and the cardinality $N$ of the set $\left\{q_{i}\right\}$. Assume that the $\left\{q_{i}\right\}$ were chosen much inside $D_{r}$, say in $D_{r / 4}$. Then, from the definition of $g$, if $r$ was chosen small enough (which doesn't influence $K$ ), on the 1.h.s. of (6.26) $g \leq\left(\frac{3 r}{4}\right)^{N}$, while on the r.h.s. $g \geq\left(\frac{3|z|}{4}\right)^{N}$, so we get

$$
\int_{B_{r / 4}} \sum_{j}\left|\sigma_{j}\right|^{2}+\left|\tau_{j}\right|^{2} \leq K \int_{B_{2 r} \backslash B_{r}}\left(\frac{r}{|z|}\right)^{2 N} \sum_{j}\left|\sigma_{j}\right|^{2}+\left|\tau_{j}\right|^{2} ;
$$

letting $N$ go to infinity, we can make the r.h.s. as small as we wish, which implies

$$
\int_{B_{r / 4}} \sum_{j}\left|\sigma_{j}\right|^{2}+\left|\tau_{j}\right|^{2}=0
$$

i.e. all the branches of the multigraph describing our original current must agree with the average on a neighbourhood of 0 . But then this average must be itself a Special Legendrian counted $Q$ times, therefore it must be smooth in this neighbourhood thanks to the basic step of the induction. We have therefore completed the proof of $\sharp_{1}$.

\section{Proof of $\sharp_{2}$ : non-accumulation of lower-order singularities}

To complete the proof of the inductive step, we have to exclude the possibility of accumulation of points in Sing $\leq Q-1$ to a singularity of order $Q$.

Let $x_{0} \in \operatorname{Sing} Q$; from Theorem 5.1 (and recalling the monotonicity formula) we can assume that we work in a ball $B^{5}$ centered at $x_{0}$ such that all the points of $C$ in this ball are of multiplicity at most $Q$ and

$$
B^{5} \cap \operatorname{Sing}^{Q}=\left\{x_{0}\right\} .
$$

By the inductive assumption, the other singularities in $B^{5}$ are isolated and of multiplicity $\leq Q-1$.

Thus we can take local coordinates about $x_{0}$ in such a way that $C$ is given by a $Q$-valued graph over $D^{2}$ that we denote by

$$
\left\{\left(\varphi_{j}(z), \alpha_{j}(z)\right)\right\}_{j=1 \cdots Q},
$$

where $z=x+i y$ is the coordinate in the Disk $D^{2}, \varphi_{i} \in \mathbb{C}$ and $\alpha_{i} \in \mathbb{R}$ and where for all $j \in\{1, \cdots, Q\}$ it holds $\left(\varphi_{j}(0), \alpha_{j}(0)\right)=(0,0)$.

Assumption on the multiplicity. In order to simplify the exposition, we assume that all smooth points of $C\left\llcorner B^{5}\right.$ have multiplicity exactly 1 . The following argument shows that there is no loss of generality in doing $\mathrm{so}^{22}$.

22 This assumption is not really needed to perform the proof presented in this last section, however it makes it less technical. 
If a smooth point $p$ has multiplicity $M \geq 2$, it must have a neighbourhood all made of smooth points of equal multiplicity $M$. Take the maximal of such neighbourhoods and denote it by $\mathcal{U}$. This smooth submanifold, counted once, constitutes an i.m. current $U$ in $B^{5}$, whose smooth points have multiplicity 1, possibly having singularities located at the same points where the singular points of $C$ were.

We claim that $U$ is a boundaryless current. Let us prove it. Let $\left\{q_{i}\right\}$ be the at most countable singularities of $C$ of order $\leq Q-1$, possibly accumulating onto 0 . First of all, from the maximality of $\mathcal{U}$ we can deduce that the topological boundary $\partial \mathcal{U}$ inside the smooth 2-dimensional submanifold $(\mathcal{C} \backslash\{0\}) \backslash \cup q_{i}$ is empty. This implies that $\partial U$ must be supported at the singularities. Thanks to this, we can localize $U$ to a neighbourhood $V_{i}^{5}$ of each isolated singularity and we can exclude the presence of boundary at each $q_{i}$ as follows. By abuse of notation we keep denoting by $U$ the localized current.

We will write $B_{\lambda}$ for the ball $B_{\lambda}^{5}\left(q_{i}\right)$. For almost any choice of $\lambda>0$, the slice of $U$ with $\partial B_{\lambda}$ exists as a 1-dimensional rectifiable current of finite mass and it is the same current, with opposite sign, as the boundary of $U_{\lambda}:=U\left\llcorner\left(V_{i}^{5} \backslash B_{\lambda}\right)\right.$. Moreover from slicing theory we have

$$
\int_{0}^{\bar{\lambda}} M\left(\partial U_{\lambda}\right) d \lambda \leq M\left(U\left\llcorner B_{\bar{\lambda}}\right) \leq M\left(C\left\llcorner B_{\bar{\lambda}}\right) .\right.\right.
$$

From the monotonicity formula and by the mean value theorem, we get the existence of a sequence $\left\{\lambda_{n}\right\} \rightarrow 0$ of positive real numbers such that

$$
M\left(\partial U_{\lambda_{n}}\right) \leq K \lambda_{n},
$$

which implies that $\partial U_{\lambda_{n}} \rightarrow 0$. On the other hand, $U_{\lambda_{n}} \rightarrow U$ since $M\left(U-U_{\lambda_{n}}\right)=$ $M\left(U\left\llcorner B_{\lambda_{n}}\right) \rightarrow 0\right.$, therefore $\partial U_{\lambda_{n}} \rightarrow \partial U$ and we get $\partial U=0$.

Once we have excluded the presence of boundary located at the singularities $q_{i}$, we can perform the same argument to exclude boundary located at 0 . So $U$ is boundaryless ${ }^{23}$.

The current $C-(M-1) U$ is thus still a Special Legendrian cycle and has exactly the same singularities as $C$; it is therefore enough to prove the result about non accumulation for this Special Legendrian "subcurrent", in order to get in for $C$. Starting now from $C-(M-1) U$, we can inductively repeat the argument and get to the desired assumption of having multiplicity 1 at all smooth points.

We still denote by $\pi$ the map on $C$ which assigns the coordinate $z$. With the assumption just discussed, the singularities of order $\leq Q-1$ are located exactly at the points $\pi^{-1}\left(z_{l}\right)$ for which $z_{l} \neq 0$ and

$$
\exists j \neq k \quad \text { s.t. } \quad\left(\varphi_{j}\left(z_{0}\right), \alpha_{j}\left(z_{0}\right)\right)=\left(\varphi_{k}\left(z_{0}\right), \alpha_{k}\left(z_{0}\right)\right) .
$$

23 An alternative argument to exclude boundary located at the singular set, is to use an analogous approximation $U_{n}$ of $U$ obtained by "cutting out" smaller and smaller balls around the singular set and show that $\partial U_{n}$ is a Cauchy sequence in the Flat-norm, therefore obtaining that $\partial U$ is a Flat 1-dimensional current. The support theorem (see [8, page 525]) tells us that a non-zero Flat 1 -current cannot be supported on a set of 0-Hausdorff dimension, therefore $\partial U=0$. 
As recalled at the beginning of this section, we are working under the assumption that the points in (7.1) form a discrete set in $D^{2} \backslash 0$, therefore at most countable. Away from them, each branch $j$ of the multiple valued graph satisfies a system ${ }^{24}$ of the form

$$
\left\{\begin{array}{l}
\partial_{\bar{z}} \varphi_{j}=v\left(\left(\varphi_{j}, \alpha_{j}\right), z\right) \partial_{z} \varphi_{j}+\mu\left(\left(\varphi_{j}, \alpha_{j}\right), z\right) \\
\nabla \alpha_{j}=h\left(\left(\varphi_{j}, \alpha_{j}\right), z\right),
\end{array}\right.
$$

where $\nu$ and $\mu$ are smooth complex valued functions on $\mathbb{R}^{5}$ such that $v(0)=$ $\mu(0)=0$ and $h$ is a smooth $\mathbb{R}^{2}$-valued map on $\mathbb{R}^{5}$.

To complete the proof of the main result we need to show

Theorem 7.1. With the previous notations, let 0 be a singular point of multiplicity $Q$ of the Special Legendrian cycle. If we are working under the (inductive) assumption that all the other singularities are of order $\leq Q-1$ and are isolated in $B^{5} \backslash\{0\}$, then there is no accumulation at 0 of singularities of the form (7.1).

The proof of the Theorem 7.1 we are giving below is inspired by the homological type argument in [21, pages 85-86]. The heuristic idea has been given in the introduction: we want to find a function that is able to "detect" the presence of isolated singularities when its topological degree is observed. Global bounds on the degree imply that it is impossible ho have a sequence of isolated singularities in Sing $\leq Q-1 C$ accumulating onto 0 .

In view of this ideas, we are now going to analyse the structure of the Special Legendrian current in a neighbourhood of an isolated singular point $q$.

The structure of an isolated singularity. Recalling our assumption on multiplicities, given an isolated singular point $q$ in $C$, for a small enough radius $\rho, C\left\llcorner B_{\rho}^{5}(q)\right.$ can be represented as

$$
C\left\llcorner B_{\rho}^{5}(q)=\oplus_{k=1}^{N} L_{k},\right.
$$

where each $L_{k}$ is either a smooth Special Legendrian embedded disk, or an immersed one branched at $q ; N$ is bounded by the multiplicity of $q$ in $C$ and $L_{k} \neq L_{l}$ if $k \neq l$.

We give a brief description of the reason why this is true. Consider the slice $\langle C,|p-q|=\rho\rangle$ : this is a smooth, one-dimensional, boundaryless current $\gamma$, so it is made of several smooth simple closed curves $\gamma_{i}$, each one counted with multiplicity 1 .

Each $\gamma_{i}$ can be obtained as the image of a circle $(\rho \cos t, \rho \sin t) \subset \mathbb{R}^{2} \equiv \mathbb{C}$ through a smooth simple map. By the smoothness assumption on all points of $\gamma_{i}$, we can get a smooth parametrization from an annulus in $\mathbb{C}$ to a subset of $C$ contained in a corresponding annulus. Take the maximal extension: since there are no other singularities, this must be a smooth simple map from $B_{\rho} \backslash\{0\}$ into $\left(C\left\llcorner B_{\rho}\right) \backslash\{q\}\right.$.

24 These are the equations we derived in (5.13) and (5.15). With respect to the notations in Sections 5 and 6, we are changing here the signs of the functions $\nu$ and $\mu$. 
By a removable singularity theorem, this map can be extended smoothly in 0 . There is no real need to invoke such a theorem: the extension to 0 is obviously continuous, and it is indeed smooth by standard elliptic theory. Thus get a smooth map from $B_{\rho}$ into $C\left\llcorner B_{\rho}\right.$; repeat the same argument for all connected components $i$ 's. A mass comparison shows that this procedure must cover the whole of $C\left\llcorner B_{\rho}\right.$.

Remark 7.2. For each branched disk $L_{k}$ in (7.3), we have a smooth parametrization from $D^{2} \subset \mathbb{C}$ into $\mathbb{R}^{5}$, with a critical point at 0 . Just like in Section 5 , by using (2.1), the calibrating condition for the Special Legendrian yields that the parametrization is a pseudo-holomorphic curve ${ }^{25}$.

By elliptic theory and conformality, as explained in [13, Section 6], one can change coordinates diffeomorhically and find that, in the new coordinates, the parametrization is of the form $\left(w^{I}, f(w)\right)$ for $w \in D^{2} \subset \mathbb{C}, f: D^{2} \rightarrow \mathbb{R}^{3}$, $I \in \mathbb{N}, I \geq 1, f(w)=o\left(|w|^{I}\right)$. However, we are not going to make use of this result.

Relative difference of branches around an isolated singularity. The following discussion is needed to understand the behaviour of the difference functions $\varphi_{i}-\varphi_{j}$ and $\alpha_{i}-\alpha_{j}$ for $i \neq j$ in a small neighbourhood of an isolated singularity $q$; let $z_{l}=\pi(q)$ and be $M$ the multiplicity of $q$. Choose a neighbourhood centered at $q$, having a cylindrical form $B_{\rho}^{2} \times B_{\rho}^{3}$, with $\rho$ small enough so that $C\left\llcorner\left(B_{\rho}^{2} \times B_{\rho}^{3}\right)\right.$ is discribed as a $M$-valued graph above $B_{\rho}^{2}\left(z_{l}\right)$, namely

$$
\left\{\left(\varphi_{j}(z), \alpha_{j}(z)\right)\right\}_{j=1 \cdots M}
$$

Remark that $\left(\varphi_{j}\left(z_{l}\right), \alpha_{j}\left(z_{l}\right)\right)$ coincide for all $j=1, \ldots, M$, while for $z \neq z_{l}$ we have $\left(\varphi_{j}(z), \alpha_{j}(z)\right) \neq\left(\varphi_{i}(z), \alpha_{i}(z)\right)$ whenever $i \neq j$ (this follows from the assumption on multiplicities taken at the beginning of this section).

Above any $z \in B_{\rho}^{2}\left(z_{l}\right) \backslash\left\{z_{l}\right\}$, consider the difference vector $\left(\left(\varphi_{i}-\varphi_{j}\right)(z),\left(\alpha_{i}-\right.\right.$ $\left.\left.\alpha_{j}\right)(z)\right) \in \mathbb{R}^{3}$ for any choice of $i \neq j$. The tail and head of this vector will belong respectively to some $L_{k}$ and $L_{l}$, possibly with $k=l$. Observe that, moving this vector by continuity for $z \neq z_{l}$, this condition on head and tail will be preserved with the same $k$ and $l$; remark that if $k=l$ the difference vector is joining two points of the same branched disk, while if $k \neq l$ it is joining points belonging to different disks. In Figure 7.1, picture on the left, there is an attempt to visualize this in the case of a single branched disk ( so $k=l$ ) that gives rise to a 2-valued graph locally around the branch point.

25 The term pseudo-holomorphic curve is commonly used for a map taking values in an almostcomplex manifold, so an even-dimensional manifold with an almost complex structure $J$ on the tangent bundle (on each tangent $J^{2}=-I d$ ). So here there is an abuse of terminology, since our parametrization takes values in $\mathbb{R}^{5}$ with a $J$ that is defined on the 4-dimensional hyperplanes of the contact distribution. However we can extend $J$ to $\mathbb{R}^{5} \times \mathbb{R}$ by setting that the vertical vector of $\mathbb{R}^{5}$ (orthogonal to the hyperplanes) is sent into the extra direction added. This gives an almost complex structure on $\mathbb{R}^{6}$, we can look at the parametrization as $\mathbb{R}^{6}$-valued, so that it becomes pseudo-holomorphic. 
For any fixed choice of $(k, l) \in\{1, \ldots, N\} \times\{1, \ldots, N\}$, we are now going to analyse the functions $\varphi_{i}-\varphi_{j}$ and $\alpha_{i}-\alpha_{j}$ for $i \neq j$ s.t.

$$
\text { ( } \left.\varphi_{i}, \alpha_{i}\right) \text { belongs to a branch of } L_{k} \text { and }\left(\varphi_{j}, \alpha_{j}\right) \text { to a branch of } L_{l},
$$

with particular interest to the behaviour of the difference vector when it evolves as described above.

This means that, in the discussion that follows, leading to Lemmas 7.5 and 7.6, we need to focus only on the disks $L_{k}$ and $L_{l}$ of (7.3).

From the second equation of the Special Legendrian system (7.2), taking differences, we get locally

$$
\nabla\left(\alpha_{i}-\alpha_{j}\right)=F \cdot\left(\varphi_{i}-\varphi_{j}\right)+G \cdot\left(\alpha_{i}-\alpha_{j}\right),
$$

where $F, G$ are bounded functions of $\left(z, \varphi_{i}(z), \varphi_{j}(z), \alpha_{i}(z), \alpha_{j}(z)\right)$ depending on the derivatives of $h$; so they satisfy $|F|,|G| \leq K_{0}<\infty$. Take a positive $\bar{t}<\frac{1}{4 K_{0}}$. In the ball $\left.\left\{\left|z-z_{l}\right| \leq \bar{t}\right)\right\}$, consider the point $w$ where $\left|\alpha_{i}-\alpha_{j}\right|$ realizes its maximum, taken over all possible choices of $i \neq j$ satisfying (7.4). Along the segment $I$ joining $z_{l}$ to $w$, we can coherently label $\alpha_{i}, \alpha_{j}, \varphi_{i}$ and $\varphi_{j}$ as smooth functions with $\alpha_{i}\left(z_{l}\right)=\alpha_{j}\left(z_{l}\right)$ and $\varphi_{i}\left(z_{l}\right)=\varphi_{j}\left(z_{l}\right)$. It makes then sense to integrate the equation (7.5) above along the segment $I$ and get

$$
\left(\alpha_{i}-\alpha_{j}\right)(w)=\int_{0}^{t}\left(\left.F\right|_{I}\right)(s)\left(\varphi_{i}-\varphi_{j}\right)(s) d s+\int_{0}^{t}\left(\left.G\right|_{I}\right)(s)\left(\alpha_{i}-\alpha_{j}\right)(s) d s
$$

for $t=|w| \leq \bar{t}$.

Notational convention. Remark that we are using $k, l$ for the fixed choice of disks in (7.3); for the branches of the $M$-valued graph describing $C\left\llcorner\left(B_{\rho}^{2} \times B_{\rho}^{3}\right)\right.$ we use, instead, the letters $i, j$. In the present discussion, we are going to denote by ||$\alpha_{i}-\alpha_{j} \|_{L^{\infty}\left(B^{2}\left(z_{l}, \bar{t}\right)\right)}$ the quantity $\sup \left\{\left|\alpha_{i}-\alpha_{j}\right|(z): z \in B^{2}\left(z_{l}, \bar{t}\right)\right.$ and $i \neq$ $j$ are as in (7.4)\}. An analogous convention holds for $\left\|\varphi_{i}-\varphi_{j}\right\|_{L^{\infty}\left(B^{2}(z l, \bar{t})\right)}$.

Thus taking the $L^{\infty}$-norm over all possible choices of $i \neq j$ satisfying (7.4) with the fixed choice of $(k, l)$, we have

$$
\begin{aligned}
\left\|\alpha_{i}-\alpha_{j}\right\|_{L^{\infty}\left(B^{2}(z l, t)\right)} & =\left|\alpha_{i}-\alpha_{j}\right|(t) \\
& \leq K_{0} t|| \varphi_{i}-\varphi_{j}\left\|_{L^{\infty}\left(B^{2}\left(z_{l}, t\right)\right)}+K_{0} t|| \alpha_{i}-\alpha_{j}\right\|_{L^{\infty}\left(B^{2}(z l, t)\right)} ;
\end{aligned}
$$

this implies

$$
\left\|\alpha_{i}-\alpha_{j}\right\|_{L^{\infty}\left(B^{2}\left(z_{l}, \bar{t}\right)\right)} \leq \frac{1}{2}\left\|\varphi_{i}-\varphi_{j}\right\|_{L^{\infty}\left(B^{2}\left(z_{l}, \bar{t}\right)\right)},
$$

with $i$ and $j$ as prescribed in (7.4). Choosing $\bar{t}$ smaller at the beginning, we can get an arbitrarily small constant instead of $\frac{1}{2}$ : therefore

$$
\frac{\left\|\alpha_{i}-\alpha_{j}\right\|_{L^{\infty}\left(B^{2}\left(z_{l}, t\right)\right)}}{\left\|\varphi_{i}-\varphi_{j}\right\|_{L^{\infty}\left(B^{2}\left(z_{l}, t\right)\right)}} \rightarrow 0 \text { as } t \rightarrow 0 .
$$


For $i \neq j$ as in (7.4), we introduce the following multivalued graph on $\{|z| \leq 1\}$, with $\rho>0$ :

$$
\left(\Theta_{i j}^{\rho}(z), \Xi_{i j}^{\rho}(z)\right)=\left(\frac{\left(\varphi_{i}-\varphi_{j}\right)\left(z_{l}+\rho z\right)}{\left\|\varphi_{i}-\varphi_{j}\right\|_{L^{\infty}\left(B^{2}\left(z_{l}, \rho\right)\right)}}, \frac{\left(\alpha_{i}-\alpha_{j}\right)\left(z_{l}+\rho z\right)}{\left\|\varphi_{i}-\varphi_{j}\right\|_{L^{\infty}\left(B^{2}\left(z_{l}, \rho\right)\right)}}\right) .
$$

Remark 7.3. This multi-valued graph has either one or two connected components. The former case happens when $k=l$, the latter when $k \neq l$. In the latter case, however, the two connected components are symmetrical with respect to $(z, 0,0)$ : one of them is just minus the other. Of course, this happens when we take $\varphi_{i}-\varphi_{j}$ and then $\varphi_{j}-\varphi_{i}$. So we can basically assume to be always dealing with a unique connected component.

We are interested in the behaviour of $\left(\Theta_{i j}^{\rho}(z), \Xi_{i j}^{\rho}(z)\right)$ as $\rho \rightarrow 0$.

Thanks to (7.6), both $\Theta_{i j}^{\rho}$ and $\Xi_{i j}^{\rho}$ are smaller or equal than 1 in modulus; more precisely $\Xi_{i j}^{\rho}$ goes uniformly to 0 as $\rho \rightarrow 0$ and $\left|\Theta_{i j}^{\rho}\right|$ always realizes the value 1 by definition. From (7.2) and (7.5), the branches of this multivalued graph solve locally on $\{0<|z| \leq 1\}$ equations of the following type:

$$
\left\{\begin{array}{l}
\bar{\partial} \Theta_{i j}^{\rho}(z)+v\left(z_{l}+\rho z\right) \partial \Theta_{i j}^{\rho}(z)+\rho S(\rho z) \Theta_{i j}^{\rho}(z)+\rho T(\rho z) \Xi_{i j}^{\rho}(z)=0 \\
\nabla \Xi_{i j}^{\rho}(z)=\rho F(\rho z) \Theta_{i j}^{\rho}(z)+\rho G(\rho z) \Xi_{i j}^{\rho}(z),
\end{array}\right.
$$

with $F, G \in L^{\infty}$ and $S, T \in L^{2}$.

We prove now:

Lemma 7.4. As $\rho \rightarrow 0$ the multi-valued graph $\left(\Theta_{i j}^{\rho}(z), \Xi_{i j}^{\rho}(z)\right)$ converges uniformly to a multi-valued graph $\left(\Theta_{i j}(z), 0\right)$, where $\Theta_{i j}$ is holomorphic in the variable $w=\sqrt{\frac{1}{1+\left|v\left(z_{l}\right)\right|^{2}}} z+v\left(z_{l}\right) \sqrt{\frac{1}{1+\left|v\left(z_{l}\right)\right|^{2}}} \bar{z}$ and homogeneous, i.e. there is $\tau \in \mathbb{Q}$ such that, for any $\lambda \in(0, \infty)$ it holds $\Theta_{i j}(\lambda z)=\lambda^{\tau} \Theta_{i j}(z)$.

Proof of Lemma 7.4. All the multivalued graphs of the sequence are pinched at 0 . By an argument similar to the one used in Theorem 6.1, we can deduce a uniform Hölder estimate on $\left(\Theta_{i j}^{\rho}(z), \Xi_{i j}^{\rho}(z)\right)$ independent of $\rho$. By Ascoli-Arzelà's theorem, as $\rho \rightarrow 0$, we can extract a subsequence converging uniformly to a multi-valued graph $\left(\Theta_{i j}(z), \Xi_{i j}(z)\right)$ and, as we said above, $\Xi_{i j}(z) \equiv 0$.

To complete the proof, we need to prove that this limit is unique, homogeneous and holomorphic in $w$.

In a way reminiscent of the discussion preceeding 7.2, the unique connected component of $\left(z,\left(\varphi_{i}-\varphi_{j}\right)\left(z_{l}+\rho z\right),\left(\alpha_{i}-\alpha_{j}\right)\left(z_{l}+\rho z\right)\right)$ (always with $i \neq j$ as in (7.4)) can be smoothly parametrized by a map from the unit disk $D^{2} \subset \mathbb{C}$ into $D^{2} \times \mathbb{C} \times \mathbb{R}$.

This can be achieved as follows. When the difference vector $\left(\varphi_{i}-\varphi_{j}, \alpha_{i}-\right.$ $\left.\alpha_{j}\right)\left(z_{l}+\rho_{0} z\right)$ (observed as an object in $\mathbb{C} \times \mathbb{C} \times \mathbb{R}$ ) evolves by continuity with a fixed 
$\rho_{0}$ as in Figure 7.1, it comes back to the starting position after that the projection of its tail onto the first $\mathbb{C}$-factor has made $I$ laps, for some integer $I$ that depends on the branching order of $L_{l}$ and $L_{k}$. So we can parametrize the multi-valued graph $\left(z,\left(\varphi_{i}-\varphi_{j}\right)\left(z_{l}+\rho_{0} z\right),\left(\alpha_{i}-\alpha_{j}\right)\left(z_{l}+\rho_{0} z\right)\right)$ restricted to $\partial D^{2} \times \mathbb{C} \times \mathbb{R}$ as a smooth curve from $\partial D^{2}$ into $\mathbb{C} \times \mathbb{C} \times \mathbb{R}$ of the form $\left(z^{I}, \phi_{l k}(z), a_{l k}(z)\right)$. Now, by the smoothness of the current out of the isolated singularity, this map can be extended to a smooth map $\left(z^{I}, \phi_{l k}(z), a_{l k}(z)\right)$ from $D^{2} \backslash\{0\}$ into $\mathbb{C} \times \mathbb{C} \times \mathbb{R}$, describing $\left\{\left(z,\left(\varphi_{i}-\varphi_{j}\right)\left(z_{l}+\rho_{0} z\right),\left(\alpha_{i}-\alpha_{j}\right)\left(z_{l}+\rho_{0} z\right)\right):|z| \leq 1\right\}$ on $\left(D^{2} \backslash\{0\}\right) \times \mathbb{C} \times \mathbb{R}$.

By a standard computation we can translate (7.7) into a first order system for $\left(\phi_{l k}, a_{l k}\right)$ of the schematic form

$$
\left\{\begin{array}{l}
\bar{\partial} \phi_{l k}(z)+\tilde{v}(z) \partial \phi_{l k}(z)+\tilde{S}(z) \phi_{l k}(z)+\tilde{T}(z) a_{l k}(z)=0 \\
\nabla a_{l k}(z)=\tilde{F}(z) \phi_{l k}(z)+\tilde{G}(z) a_{l k}(z)=0
\end{array}\right.
$$

with $C^{1, \sigma}$ coefficients $(0<\sigma<1)$ and $|\tilde{v}|$ small. Elliptic regularity yields that the extension of $\left(z^{I}, \phi_{l k}(z), a_{l k}(z)\right)$ to $D^{2}$, which is obviously continous at 0 , is actually at least $C^{2}$.

Moreover, after the linear change of coordinates $z \rightarrow w$

$$
w=\sqrt{\frac{1}{1+\left|v\left(z_{l}\right)\right|^{2}}} z+v\left(z_{l}\right) \sqrt{\frac{1}{1+\left|v\left(z_{l}\right)\right|^{2}}} \bar{z},
$$

and by taking the $\partial_{w}$-derivative of the first equation, we get an inequality of the form

$$
\left|\tilde{\Delta} \phi_{l k}\right|(w) \leq K\left|D \phi_{l k}\right|(w)+K\left|\phi_{l k}\right|(w),
$$

where $K$ is a positive constant and $\tilde{\Delta}$ is an elliptic second order operator that coincides with the Laplacian for $w=0$. By elliptic theory, the function $f(\rho):=$ $\left\|\phi_{l k}\right\|_{L^{\infty}\left(B_{\rho}^{2}\right)}$ cannot have derivatives at 0 all vanishing, see [13, Theorem 1.1 and Corollary 1 on page 41] (this theorem is basically due to Hartman and Wintner).

Fix $z \in \partial D^{2} \subset \mathbb{C}$. Then, since $f(\rho)$ is just $\left\|\varphi_{i}-\varphi_{j}\right\|_{L^{\infty}\left(B^{2}\left(z_{l}, \rho\right)\right)}$, we can write

$$
\Theta_{i j}(z):=\lim _{\rho \rightarrow 0} \Theta_{i j}^{\rho}(z)=\lim _{\rho \rightarrow 0} \frac{\left(\varphi_{i}-\varphi_{j}\right)\left(z_{l}+\rho z\right)}{f(\rho)}
$$

and

$$
\Theta_{i j}(\lambda z):=\lim _{\rho \rightarrow 0} \Theta_{i j}^{\rho}(\lambda z)=\lim _{\rho \rightarrow 0} \frac{\left(\varphi_{i}-\varphi_{j}\right)\left(z_{l}+\rho \lambda z\right)}{f(\rho)} .
$$

This blow-up can be equivalently expressed in terms of $\phi_{l k}$. What we are looking for in (7.9) and (7.10) are respectively $\Phi_{l k}(z):=\lim _{\rho \rightarrow 0} \frac{\phi_{l k}(\rho z)}{f(\rho)}$ and $\Phi_{l k}(\lambda z)=$ $\lim _{\rho \rightarrow 0} \frac{\phi_{l k}(\lambda \rho z)}{f(\rho)}$. As we saw above, the function $f$ is smooth and it is not possible that all of its derivatives at $\rho=0$ vanish. 
It is then enough to restrict to the segment joining $z_{l}$ to $z$ and apply de L'Hôpital's theorem to compute the two limits: we get that there is $k \in \mathbb{N}$, namely the first integer such that $f^{(k)}(0) \neq 0$, for which $\Phi(\lambda z)=\lambda^{k} \Phi(z)$. This immediately gives $\Theta_{i j}(\lambda z)=\lambda^{\tau} \Theta_{i j}(z)$ for $\tau=\frac{k}{Q}$ and the uniqueness of the limit.

Moreover, it is not difficult to see that the convergence $\Theta_{i j}^{\rho} \rightarrow \Theta_{i j}$ is more that just uniform: indeed, the gradients are equibounded and equicontinuous, so we can pass (7.7) to the limit and get that $\left(\Theta_{i j}(z), \Xi_{i j}(z)\right)$ must solve, locally on $\{0<|z| \leq 1\}$,

$$
\left\{\begin{array}{l}
\bar{\partial} \Theta_{i j}(z)+v\left(z_{l}\right) \partial \Theta_{i j}(z)=0, \text { with }\left|v\left(z_{l}\right)\right| \ll 1, \\
\nabla \Xi_{i j}(z)=0 .
\end{array}\right.
$$

Therefore, since $\left(\Theta_{i j}(0), \Xi_{i j}(0)\right)=(0,0)$, from the second equation we recover once again $\left(\Theta_{i j}(z), \Xi_{i j}(z)\right)$ must be of the form $\left(\Theta_{i j}(z), 0\right)$. Consider now the equation for $\Theta_{i j}$ : again with the linear change of complex variable $z \rightarrow w$, we can deduce that $\Theta_{i j}$ solves

$$
\frac{\partial}{\partial \bar{w}} \Theta_{i j}(w)=0 ;
$$

thus $\Theta_{i j}$ is holomorphic w.r.t. the variable $w$. We will also say that it is almostholomorphic in $z$.

The fact that $\Theta_{i j}$ is holomorphic in $w$ and homogeneous implies that $\Theta_{i j}$ is always non-zero on $\partial D^{2}$. Indeed, if we had a zero on $y \in \partial D^{2}, \Theta_{i j}$ would be zero on the whole segment joining 0 to $y$ : recalling that there is a unique connected component and by holomorphicity we would then get that $\Theta_{i j}$ is zero on the whole of $D^{2}$, contradicting that its $L^{\infty}$-norm is 1 .

Lemma 7.5. Fix $(k, l) \in\{1, \ldots, N\} \times\{1, \ldots, N\}$; for $i \neq j$ s.t. $\left(\varphi_{i}, \alpha_{i}\right)$ belongs to a branch of $L_{k}$ and $\left(\varphi_{j}, \alpha_{j}\right)$ to a branch of $L_{l}$ (possibly with $k=l$ ), the following holds: for any $\delta>0$ there is $\rho>0$ small enough, s.t.

$$
\left|z-z_{l}\right|<\rho \Rightarrow \frac{\left|\alpha_{i}-\alpha_{j}\right|^{2}}{\left|\varphi_{i}-\varphi_{j}\right|^{2}+\left|\alpha_{i}-\alpha_{j}\right|^{2}}(z)<\delta .
$$

In particular, for $\left|z-z_{l}\right|<\rho$ and $z \neq z_{l}$, we have $\varphi_{i}-\varphi_{j} \neq 0$ for $i \neq j$.

Proof of Lemma 7.5. By contradiction, if for some $\delta>0$ and a sequence $z_{n} \rightarrow z_{l}$ we had $\frac{\left|\alpha_{i}-\alpha_{j}\right|^{2}}{\left|\varphi_{i}-\varphi_{j}\right|^{2}+\left|\alpha_{i}-\alpha_{j}\right|^{2}}\left(z_{n}\right) \geq \delta$, the sequence

$$
\left(\Theta_{i j}^{\left|z_{n}-z_{l}\right|}(z), \Xi_{i j}^{\left|z_{n}-z_{l}\right|}(z)\right)
$$

could not converge to a limit of the form $\left(\Theta_{i j}(z), 0\right)$. 
Lemma 7.6. Fix $(k, l) \in\{1, \ldots, N\} \times\{1, \ldots, N\}$; by Lemma 7.5, for $\rho$ small enough and for $i \neq j$ s.t. $\left(\varphi_{i}, \alpha_{i}\right)$ belongs to a branch of $L_{k}$ and $\left(\varphi_{j}, \alpha_{j}\right)$ to a branch of $L_{l}$ (possibly with $k=l$ ), it makes sense to compute the degree of

$$
\frac{\varphi_{i}-\varphi_{j}}{\left|\varphi_{i}-\varphi_{j}\right|}
$$

on the closed curve $\gamma=L_{l} \cap \pi^{-1}\left\{\left|z-z_{l}\right|=\rho\right\}$. This degree is strictly positive.

Proof of Lemma 7.6. See Figure 7.1 for a visual explanation. $\gamma$ is a closed, connected curve; orient it so that its projection $\pi(\gamma)$ on $\mathbb{C}$ winds positively. Fix then, with an arbitrary starting point on $\gamma$, any determination of the vector $\varphi_{i}-\varphi_{j}$ and let it evolve along $\gamma$ in the given direction, keeping its tail on the curve; meanwhile, its head will move along a closed curve in $L_{k}$, which could be either the same or a different curve. In the former case we are staying inside the same branched disk $L_{l}$, in the latter we are dealing with two different disks $L_{k}$ and $L_{l}$. In any case, the vector will eventually come back to the initial one after having run, possibly more than once (say $I$ times), over the whole of $\gamma$. We then get a smooth map $\frac{\varphi_{i}-\varphi_{j}}{\left|\varphi_{i}-\varphi_{j}\right|}$ from a multiple cover $\gamma \oplus \ldots \oplus \gamma$ of $\gamma$ to $S^{1}$. The multiple cover is homeomorphic to $S^{1}$, so it makes sense to consider the degree of the $S^{1}$-valued map $\frac{\varphi_{i}-\varphi_{j}}{\left|\varphi_{i}-\varphi_{j}\right|}$ on $\gamma \oplus \ldots \oplus \gamma$. Introduce the multi-valued graph $\varphi_{i}-\varphi_{j}$ for $i, j$ in the $L_{k}$ and $L_{l}$ involved. This multi-valued graph has a unique connected component (or two symmetrical ones). By Lemma 7.4

$$
\frac{\varphi_{i}-\varphi_{j}}{\left|\varphi_{i}-\varphi_{j}\right|}\left(z_{l}+\rho z\right)=\frac{\Theta_{i j}^{\rho}}{\left|\Theta_{i j}^{\rho}\right|}(z) \rightarrow \frac{\Theta_{i j}}{\left|\Theta_{i j}\right|}(z)
$$

uniformly, so it must contribute with a strictly positive degree on $\gamma \oplus \ldots \oplus \gamma$ if $\rho$ was small enough, since so happens $\Theta_{i j}$, which is almost-holomorphic.

Some heuristics. Roughly speaking, with Lemmas 7.5 and 7.6 we have found out that, by observing the relative differences between branches, we can somehow "count" the points in Sing $\leq Q-1$.

Indeed, locally around each $q \in \operatorname{Sing} \leq Q-1$, we have functions defined via the relative differences of branches that are able to catch the presence of $p$ by producing a strictly positive integer contribution when the degree is observed.

However, both in the definition of these functions $\frac{\varphi_{i}-\varphi_{j}}{\left|\varphi_{i}-\varphi_{j}\right|}$ and in the proof of the strict positiveness of the degree, we made a key use of the structure (7.3) of $C$ around an isolated singularity $q$. This allowed us, locally around $q$, a "separation of the branches": we were able to focus just on the disks $L_{l}$ and $L_{k}$ involved in the evolution of the difference vector. With the use of PDEs and parametrizations, we were lead to the results on $\Theta_{i j}$ and to the control on the degree.

Moreover we have produced a way to "count' singularities with functions that are only defined close enough to the singular point itself. As we get further from the 


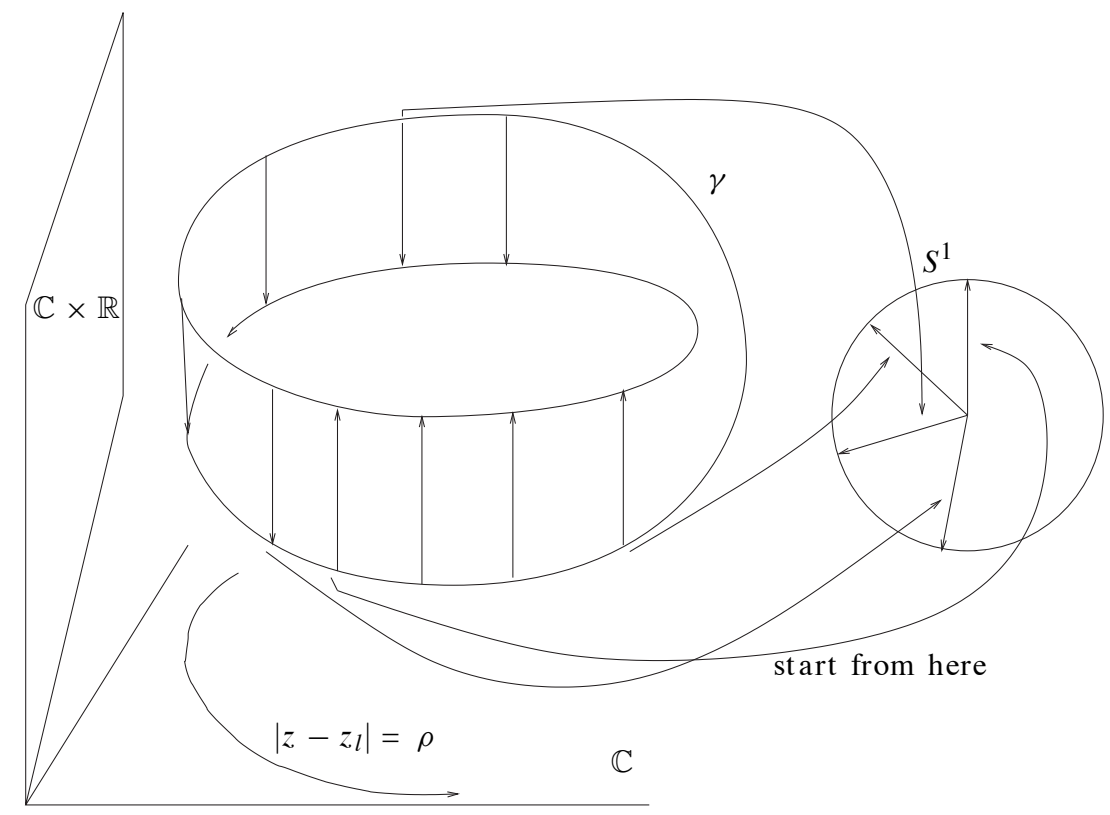

Figure 7.1. On the left, an attempt to represent $\gamma=\pi^{-1}\left\{\left|z-z_{l}\right|=\rho\right\}$ in the case of a two-valued graph in $\mathbb{C} \times \mathbb{C} \times \mathbb{R}$. We observe the evolution of the difference vector joining points above the same $z \in\left\{\left|z-z_{l}\right|=\rho\right\} \subset \mathbb{C}$ : as the tail of the difference vector runs along $\gamma$, we keep track (picture on the right) of the normalized difference vector projected on the second $\mathbb{C}$-factor an observe how it winds around $S^{1}$. If we are around an isolated singularity, then we find that the represented map from $\gamma$ to $S^{1}$ has strictly positive degree. In this particular picture, after having run once along $\gamma$ the normalized difference vector winds once positively around $S^{1}$.

singularity it might happen that the difference vector $\left(\varphi_{i}-\varphi_{j}, \alpha_{i}-\alpha_{j}\right)(z)$ has zero $\mathbb{C}$-component, so we cannot construct a global function that counts singularities by looking only at $\varphi_{i}-\varphi_{j}$.

With the notations taken at the beginning of this section, we are thus lead to the following questions:

- can we produce a similar function that is well-defined in a whole neighbourhood of $0 \in D^{2}$ and whose degree still detects the presence of points in Sing $\leq Q-1$ ?

- can we find a lower bound for the degree of this function, to allow a homotopy argument as sketched in the introduction?

Remark that we have no information about the structure of $C$ around the origin, unlike it happened in the situation (7.3). A natural candidate function on $D^{2}$ to collect the information on the degree of the difference between branches would be $\left(\Pi_{i \neq j}\left(\varphi_{i}-\varphi_{j}\right), \Pi_{i \neq j}\left(\alpha_{i}-\alpha_{j}\right)\right)$. However this function does not solve any appealing equation. 
To overcome this difficulty we are going to introduce a new 2-dimensional space $\pi^{*} C$ that is modelled on the current $C$ but allows to observe the relative difference of branches without having to separate them and, most important, with the use of this new space we will be able to write equations for the difference of branches: this will be crucial in answering the second question.

More precisely, due to dimensional reasons rooted in the problem, we will produce a function $u$ on the space $\pi^{*} C \times \mathbb{R}$ taking values in $\mathbb{C} \times \mathbb{R}$ : this function will mimic the behaviour of the difference vector when we are close enough to an isolated singularity.

Recalling the heuristic ideas from the introduction, we can see that the technical reason is that we need a function $u$ that vanishes exactly at the singular points and for which we can take the degree of $\frac{u}{|u|}$ : since the difference of branches is naturally an element of $\mathbb{C} \times \mathbb{R}$, we need to add an extra-dimension to $\pi^{*} C$ in order to have a notion of topological degree.

The core of the proof of Theorem 7.1 will be Lemma 7.8, where we bound from below the degree of $\frac{u}{|u|}$. Lemma 7.7 is a restatement of Lemma 7.6 in terms of the new space $\pi^{*} C \times \mathbb{R}$ and of the function $u$. These two results together allow the homological argument that yields Theorem 7.1.

Proof of the non-accumulation. Denote by $\pi^{*} C$ the following subset of $\mathbb{R}^{3} \times$ $\mathbb{R}^{3} \times D^{2}$ :

$$
\pi^{*} C:=\left\{\begin{array}{c}
\xi=\left(\zeta_{1}, \zeta_{2}, z\right) \in \mathbb{R}^{3} \times \mathbb{R}^{3} \times D^{2} \quad \text { s.t. } \quad \exists j, k \in\{1 \cdots Q\} \\
\text { satisfying } \zeta_{1}=\left(\varphi_{j}(z), \alpha_{j}(z)\right) \quad \text { and } \quad \zeta_{2}=\left(\varphi_{k}(z), \alpha_{k}(z)\right)
\end{array}\right\}
$$

By an abuse of notation we will also write $\zeta_{1}=\left(\varphi_{1}, \alpha_{1}\right)$ and $\zeta_{2}=\left(\varphi_{2}, \alpha_{2}\right)$, moreover $^{26}$ we denote $z=\pi(\xi)$ - i.e. $\pi$ is extended naturally to $\pi^{*} C$.

Observe that $C \subset \pi^{*} C$ as the result of the identification of $C$ with the points $\left(\zeta_{1}, \zeta_{2}, z\right)$ such that $\zeta_{1}=\zeta_{2}$. Away from these points, $\pi^{*} C \backslash C$ realizes a smooth 2-dimensional oriented submanifold of $\mathbb{R}^{3} \times \mathbb{R}^{3} \times D^{2}$ with local chart given by $z$.

On $\pi^{*} C$ we define the function

$$
d(\xi):=\left|\zeta_{1}-\zeta_{2}\right|=\sqrt{\left|\alpha_{1}-\alpha_{2}\right|^{2}+\left|\varphi_{1}-\varphi_{2}\right|^{2}},
$$

which is smooth and non-zero on $\pi^{*} C \backslash C$. On $\pi^{*} C \backslash C$ we define

$$
\Delta(\xi):=\frac{\left|\alpha_{1}-\alpha_{2}\right|^{2}}{\left|\alpha_{1}-\alpha_{2}\right|^{2}+\left|\varphi_{1}-\varphi_{2}\right|^{2}} .
$$

Let $\phi$ be a smooth non negative compactly supported function satisfying

$$
\phi(s)= \begin{cases}1 & \text { for } s<1, \\ 0 & \text { for } s>2 .\end{cases}
$$

For $1>\delta>0$ we denote $\phi_{\delta}(\cdot)=\phi(\cdot / \delta)$.

${ }^{26}$ Here $\zeta_{i}(i \in\{1,2\})$ will always be an element of $\mathbb{R}^{3}$ of the form $\left(\varphi_{j}(z), \alpha_{j}(z)\right)$; it should not be confused with the complex coordinate $\zeta$ in $\mathbb{C}_{z} \times \mathbb{C}_{\zeta} \times \mathbb{R}_{a}$ used in Sections 5 and 6 , which will anyway not appear in this section. 
Let $\delta<1$ be a regular value of the function $\Delta$ on $\pi^{*} C \backslash C$ we define a stretching-contracting map

$$
S_{\delta}: \mathbb{R}^{3} \longrightarrow \mathbb{R}^{3}
$$

in the following way : $S_{\delta}$ is axially symmetric about the $z$-axis, $\left|S_{\delta}(x, y, z)\right|=$ $|(x, y, z)|$ and the following conditions are satisfied:

$$
S_{\delta}(x, y, z)= \begin{cases}S_{\delta}(x, y, z)=\operatorname{sgn}(z)\left(0,0, \sqrt{x^{2}+y^{2}+z^{2}}\right) & \text { if } \frac{z^{2}}{x^{2}+y^{2}+z^{2}}>\delta, \\ S_{\delta}(x, y, z)=(x, y, z) & \text { if } \frac{z^{2}}{x^{2}+y^{2}+z^{2}}<\frac{\delta}{2},\end{cases}
$$

with a smooth join for $\frac{\delta}{2} \leq \frac{z^{2}}{x^{2}+y^{2}+z^{2}} \leq \delta$ chosen so to ensure $\operatorname{det}\left(D S_{\delta}\right)>0$.

Denote by $N$ the following 3-dimensional manifold:

$$
N:=\left\{(\xi, t) \in\left(\pi^{*} C \backslash C\right) \times \mathbb{R}\right\} .
$$

Set

$$
D=D_{\delta}:=\frac{1}{\sqrt{\frac{1}{\delta}-1}} .
$$

Observe that $D>0$ has been chosen in particular in such a way that

$$
D^{-1}\left|\alpha_{1}-\alpha_{2}\right| \leq\left|\varphi_{1}-\varphi_{2}\right| \Longleftrightarrow \Delta(\xi) \leq \delta \Longleftrightarrow \phi_{\delta}(\Delta(\xi))=1 .
$$

At this stage we are going to make a short digression to choose a suitable value for $\delta<1$ (besides the requirement that $\delta$ be a regular value of $\Delta$ ), which will be kept throughout the rest of the section.

Let $R$ be the radius of $D^{2}$. Denote by $B_{r}$, for $r \leq R$, the part of $\pi^{*} C \backslash C$ above the set $\{|z|<r\}, B_{r}:=\pi-1\left(B_{r}^{2}(0)\right)$. For any $\delta<1$, express the set $\{\Delta>\delta\}$ as the union of its connected components, i.e. $\{\Delta>\delta\}=\cup_{i} A_{\delta}^{i}$. We are going to prove the following claim: there exist $\delta<1$ and $\bar{r}<R$ s.t.

$$
\forall i \quad \text { and } \forall r \leq \bar{r} \quad A_{\delta}^{i} \cap \partial B_{r} \neq \emptyset \Rightarrow A_{\delta}^{i} \cap \partial B_{R}=\emptyset .
$$

To prove the claim, we argue by contradiction: assume the existence of sequences $\delta_{n} \rightarrow 1, r_{n} \rightarrow 0$ for which we can always find a connected component intersecting both $\partial B_{r_{n}}$ and $\partial B_{R}$. Then we can choose $C^{1}$ curves $\gamma_{n}$, parametrized by arc length, joining $\partial B_{r_{n}}$ to $\partial B_{R}$ and staying inside the corresponding connected component. Up to a subsequence, by Ascoli-Arzelà's theorem, we can assume the existence of a uniform limit curve $\gamma$, joining 0 to $\partial B_{R}$. The function $\Delta$ is greater than $\delta_{n}$ on the image of $\gamma_{n}$, therefore

$$
\delta_{n} \rightarrow 1 \Rightarrow \Delta \circ \gamma \equiv 1 \Rightarrow\left|\varphi_{1}-\varphi_{2}\right| \rightarrow 0 \text { as } n \rightarrow \infty .
$$


The limit curve $\gamma$ could a priori be merely continuous and not $C^{1}$. We can write, from (7.5), for any $n$ and for any $t$ in the domain of $\gamma_{n}$ :

$$
\left|\alpha_{1}-\alpha_{2}\right|\left(\gamma_{n}(t)\right) \leq\left|\varphi_{1}-\varphi_{2}\right|\left(\gamma_{n}(0)\right)+K_{0} \int_{0}^{t}\left|\alpha_{1}-\alpha_{2}\right|\left(\gamma_{n}(s)\right) d s .
$$

Sending to the limit as $n \rightarrow \infty$

$$
\left|\alpha_{1}-\alpha_{2}\right|(\gamma(t)) \leq K_{0} \int_{0}^{t}\left|\alpha_{1}-\alpha_{2}\right|(\gamma(s)) d s,
$$

thus $\alpha_{1}-\alpha_{2}$ is identically 0 on the curve $\gamma$; here $\varphi_{1}-\varphi_{2}$ also vanishes and therefore the image of $\gamma$ is a line of singularities, contradiction. Thus the claim is proved. End of the digression.

Now, for the $\delta$ just chosen, take any positive $r \leq \bar{r}$ arbitrarily small and such that $\pi^{-1}\left(\partial B_{r}^{2}(0)\right)$ does not intersect the set of $z_{l}$ satisfying (7.1). Let

$$
\varepsilon_{0}:=\inf \left\{\frac{d(\xi)}{\sqrt{1+D^{2}}} ; \xi \in\left(\pi^{*} C \backslash C\right) \cap \pi^{-1}\left(\partial B_{r}^{2}(0)\right)\right\} .
$$

By the assumption on $r, \varepsilon_{0}>0$.

Let $\varepsilon>0$ be a regular value less than $\varepsilon_{0}$ for the function $\left|\varphi_{1}-\varphi_{2}\right|$. Denote by $g$ the following function on $\pi^{*} C \backslash C$ :

$$
g(\xi):=\frac{\varphi_{1}-\varphi_{2}}{\max \left\{\left|\alpha_{1}-\alpha_{2}\right|, D \varepsilon\right\}}
$$

Observe that since $\left(C\left\llcorner B^{5}\right) \backslash\{0\}\right.$ is assumed to be a smooth Special Legendrian curve and since $\left(\varphi_{j}(0), \alpha_{j}(0)\right)=(0,0)$ for all $j,\left|\varphi_{1}-\varphi_{2}\right|^{-1}(\{\varepsilon\})$ is a smooth compact curve in $\pi^{*} C \backslash C$ for any regular value $\varepsilon>0$. Observe moreover that since $\varepsilon<\varepsilon_{0}$ we have that

$$
\left(\pi^{*} C \backslash\{\xi ; \Delta(\xi)>\delta\}\right) \cap\left(\left|\varphi_{1}-\varphi_{2}\right|^{-1}(\{\varepsilon\})\right) \cap \pi^{-1}\left(\partial B_{r}^{2}(0)\right)=\emptyset .
$$

Define the open set $U$ in $\pi^{*} C \backslash C$ made of the connected components of $\{\Delta>\delta\}$ that intersect $B_{r}=\pi^{-1}\left(B_{r}^{2}(0)\right)$ (and therefore not $\partial B_{R}$ thanks to (7.13)).

For any fixed $r \leq \bar{r}$, choose $\varepsilon$ small enough as follows: firstly, $\varepsilon<\varepsilon_{0}$; secondly, take

$$
\varepsilon<\min \left\{\left|\varphi_{1}-\varphi_{2}\right|(\xi): \xi \in \overline{\partial\left(U \cap\left(B_{R} \backslash B_{r}\right)\right) \backslash \partial B_{r}} \subset \partial U\right\} .
$$

The minimum on the r.h.s. is strictly positive. Indeed, if it were 0 , then either we would have a singular point that realizes it, or a smooth point where $\Delta=1$. In the former case, Lemma 7.5 tells us that there is a neighbourhood of the singularity where $\left\{\Delta<\frac{\delta}{2}\right\}$, therefore it cannot be a boundary point of $U$, since in $U$ we have 
$\Delta>\delta$. In the latter case there ought to be a neighbourhood where $\{\Delta>\delta\}$, so it could not be a boundary point.

Finally define the open set in $\pi^{*} C \backslash C$

$$
\Sigma_{\varepsilon, r}=\left(\left\{\left|\varphi_{1}-\varphi_{2}\right|<\varepsilon\right\} \cap B_{r}\right) \cup U .
$$

$\Sigma_{\varepsilon, r}$ has the following properties:

(i) $z_{l} \in \pi\left(\Sigma_{\varepsilon, r}\right) \Rightarrow z_{l} \in \pi\left(B_{r}\right)$, since there are no singularities in $U$ due to Lemma 7.5;

(ii) $p \in \partial \Sigma_{\varepsilon, r} \Rightarrow\left\{\begin{array}{l}\left|\varphi_{1}-\varphi_{2}\right|(p)=\varepsilon \\ \Delta(p) \leq \delta\end{array} \quad\right.$ or $\left\{\begin{array}{l}\left|\varphi_{1}-\varphi_{2}\right|(p) \geq \varepsilon \\ \Delta(p)=\delta,\end{array}\right.$ so $|g| \equiv \sqrt{\frac{1}{\delta}-1}=D^{-1}$ on $\partial \Sigma_{\varepsilon, r}$.

Thus $\delta$ and $\varepsilon$ have been chosen in such a way that $\partial \Sigma_{\varepsilon, r}$ is a closed smooth compact curve in $\pi^{*} C \backslash C$ which is included in the level set $|g|^{-1}\left(\left\{D^{-1}\right\}\right)$. Remark that $\partial \Sigma_{\varepsilon, r}$ is obtained by homotopy from the loop $\pi^{-1}\{|z|=r\}$ without crossing any singularity of $C \subset \pi^{*} C$.

On $N$ we define the map $v$ given by

$$
\begin{aligned}
v & : N \longrightarrow S^{2} \\
& (\xi, t) \longrightarrow \frac{\left(g(\xi), \alpha_{1}-\alpha_{2}+t \phi_{\delta} \circ \Delta(\xi)\right)}{\sqrt{|g(\xi)|^{2}+\left|\alpha_{1}-\alpha_{2}+t \phi_{\delta} \circ \Delta(\xi)\right|^{2}}} .
\end{aligned}
$$

Observe that $|g(\xi)|^{2}+\left|\alpha_{1}-\alpha_{2}+t \phi_{\delta} \circ \Delta(\xi)\right|^{2}=0$ implies that $\left|\varphi_{1}-\varphi_{2}\right|=0$. If $\alpha_{1}-\alpha_{2} \neq 0$ then $\phi_{\delta} \circ \Delta(\xi)=0$ and hence we would have $\left|\alpha_{1}-\alpha_{2}\right|=0$ which is a contradiction. Hence $v$ is well-defined smooth map on $N$. Finally define the $S^{2}$-valued map $u$ by

$$
u:=S_{\delta} \circ v: N \rightarrow S^{2} .
$$

On the complement of $\Sigma_{\varepsilon, r}$ the map $v$ simplifies to

$$
v(\xi)=\frac{\left(g(\xi), \alpha_{1}-\alpha_{2}+t\right)}{\sqrt{|g(\xi)|^{2}+\left|\alpha_{1}-\alpha_{2}+t\right|^{2}}} .
$$

From the definition of $S_{\delta}$, for any two-form $\omega$ on $S^{2}$ we have hence that, on $N \backslash$ $\left(\Sigma_{\varepsilon, r} \times \mathbb{R}\right),\left(S_{\delta} \circ v\right)^{*} \omega=0$ for $|t|>1 / \varepsilon$ (Assuming without loss of generality that $d(\xi)$ is bounded by 1 on $\pi^{*} C$ ). Hence the degree of $u$ restricted to any closed compact curve in the complement of $\Sigma_{\varepsilon, r}$ times $\mathbb{R}$ is well defined since in $N \backslash$ $\left(\Sigma_{\varepsilon, r} \times \mathbb{R}\right)$ we have $u^{*} \omega \neq 0$ only on a compact set.

The rest of the section is occupied with the proof of the following two lemmas, which will imply by a simple homotopy argument that can be found at the end of the section, that the number of $z_{l}$ is uniformly bounded and Theorem 7.1 will be proved. 
Lemma 7.7. For any $z_{l}$ as in (7.1) and for $\rho>0$ small enough

$$
\int_{\pi^{-1}\left(\partial B_{\rho}^{2}\left(z_{l}\right)\right) \times \mathbb{R}} u^{*} \omega \geq 1,
$$

where $\omega$ is an arbitrary 2-form on $S^{2}$ such that $\int_{S^{2}} \omega=1$.

Lemma 7.8. Under the previous notations, there exists a constant $K \in \mathbb{R}^{+}$independent of $r$ and $\varepsilon$ such that (the indexes of the two-form are to be understood $\bmod 3$ )

$$
\int_{\partial \Sigma_{\varepsilon, r} \times \mathbb{R}} u^{*}\left(\sum_{i=1}^{3} x^{j} d x^{j+1} \wedge d x^{j-1}\right) \geq-K .
$$

Proof of Lemma 7.8. This constitutes the core of the proof of Theorem 7.1.

Recall that $|g(\xi)| \equiv D^{-1}$ on $\partial \Sigma_{\varepsilon, r}$. Denote $\lambda$ the following function on $\Sigma_{\varepsilon, r} \times \mathbb{R}$

$$
\lambda(\xi, t):=\sqrt{D^{-2}+\left(\alpha_{1}-\alpha_{2}+t\right)^{2}} .
$$

We additionally denote by $w$ the following $\mathbb{C} \times \mathbb{R}$-valued map ${ }^{27}$ on $\Sigma_{\varepsilon, r} \times \mathbb{R}$ :

$$
w(\xi, t):=\frac{\left(g(\xi), \alpha_{1}-\alpha_{2}+t\right)}{\lambda} .
$$

Observe that $w=v$ on $\partial \Sigma_{\varepsilon, r} \times \mathbb{R}$.

First we claim that

$$
\int_{\Sigma_{\varepsilon, r} \times \mathbb{R}}\left|\left(S_{\delta} \circ w\right)^{*}\left(d x^{1} \wedge d x^{2} \wedge d x^{3}\right)\right| d \mathcal{H}^{2} d t\llcorner N<+\infty .
$$

We now prove the claim (7.18). We write on one hand

$$
S_{\delta}^{*}\left(d x^{1} \wedge d x^{2} \wedge d x^{3}\right)=\operatorname{det}\left(D S_{\delta}\right)(y) d y^{1} \wedge d y^{2} \wedge d y^{3}
$$

and locally on the other hand

$$
\begin{aligned}
w^{*}\left(d y^{1} \wedge d y^{2} \wedge d y^{3}\right)= & \lambda^{-3} d f^{1} \wedge d f^{2} \wedge d\left(\alpha_{1}-\alpha_{2}+t\right) \\
& +\lambda^{-2} d \lambda^{-1} \wedge\left(f^{1} d f^{2}-f^{2} d f^{1}\right) \wedge d\left(\alpha_{1}-\alpha_{2}+t\right) \\
& +\lambda^{-2}\left(\alpha_{1}-\alpha_{2}+t\right) d f^{1} \wedge d f^{2} \wedge d \lambda^{-1}
\end{aligned}
$$

where ${ }^{28}$ locally $f(z)=f^{1}(z)+i f^{2}(z):=g^{1}(\xi(z))+i g^{2}(\xi(z))$. Observe now that the following 3 - and 2 -forms are zero

$$
d f^{1} \wedge d f^{2} \wedge d\left(\alpha_{1}-\alpha_{2}\right) \equiv 0 \quad \text { and } \quad d \lambda^{-1} \wedge d\left(\alpha_{1}-\alpha_{2}+t\right) \equiv 0 .
$$

${ }^{27}$ Sometimes we will also look at $w$ as a $\mathbb{R}^{3}$-valued map.

$28 g^{1}$ and $g^{2}$ denote respectively the real and imaginary part of $g$. 
Hence (7.19) becomes, from the definition of $\lambda$,

$$
\begin{aligned}
w^{*}\left(d y^{1} \wedge d y^{2} \wedge d y^{3}\right)= & \lambda^{-3} d f^{1} \wedge d f^{2} \wedge d t \\
& -\lambda^{-5}\left(\alpha_{1}-\alpha_{2}+t\right)^{2} d f^{1} \wedge d f^{2} \wedge d t \\
= & \lambda^{-5} D^{-2} d f^{1} \wedge d f^{2} \wedge d t
\end{aligned}
$$

We rewrite

$$
w^{*}\left(d y^{1} \wedge d y^{2} \wedge d y^{3}\right)=\frac{i}{2} \lambda^{-5} D^{-2}\left[\left|\partial_{z} f\right|^{2}-\left|\partial_{\bar{z}} f\right|^{2}\right] d z \wedge d \bar{z} \wedge d t .
$$

We first estimate the following integral:

$$
\int_{-\infty}^{+\infty} \operatorname{det}\left(D S_{\delta}\right)(w(\xi, t)) \lambda^{-5} d t \leq C_{\delta} \int_{-\infty}^{+\infty} \frac{d \tau}{\left(D^{-2}+\tau^{2}\right)^{\frac{5}{2}}} \leq C_{\delta} .
$$

Observe that

$$
|\nabla f| \leq \varepsilon^{-1} D^{-1}\left|\nabla\left(\varphi_{1}-\varphi_{2}\right)\right|+\varepsilon^{-2} D^{-2}\left|\varphi_{1}-\varphi_{2}\right|\left|\nabla\left(\alpha_{1}-\alpha_{2}\right)\right| .
$$

Since $\int_{D^{2}} \sum_{j=1}^{Q}\left(\left|\nabla \varphi_{j}\right|^{2}+\left|\nabla \alpha_{j}\right|^{2}\right)<+\infty$ combining (7.20), (7.23) and (7.24) we obtain the claim (7.18).

We now establish the lower bound (7.17). To that purpose we compute an equation for $f$.

From the equations in (7.2) we deduce that locally

$$
\left\{\begin{aligned}
\partial_{\bar{z}}\left(\varphi_{1}-\varphi_{2}\right)= & v\left(\varphi_{2}, \alpha_{2}\right) \partial_{z}\left(\varphi_{1}-\varphi_{2}\right)+\left[v\left(\varphi_{1}, \alpha_{1}\right)-v\left(\varphi_{2}, \alpha_{2}\right)\right] \partial_{z} \varphi_{1} \\
& +\mu\left(\varphi_{1}, \alpha_{1}\right)-\mu\left(\varphi_{2}, \alpha_{2}\right) \\
\nabla\left(\alpha_{1}-\alpha_{2}\right)= & h\left(\varphi_{1}, \alpha_{1}\right)-h\left(\varphi_{2}, \alpha_{2}\right)
\end{aligned}\right.
$$

We have that

$$
\partial_{\bar{z}} f=\frac{\partial_{\bar{z}}\left(\varphi_{1}-\varphi_{2}\right)}{\max \left\{\left|\alpha_{1}-\alpha_{2}\right|, D \varepsilon\right\}}-f \mathbf{1}_{\left|\alpha_{1}-\alpha_{2}\right|>D \varepsilon} \frac{\partial_{\bar{z}}\left|\alpha_{1}-\alpha_{2}\right|}{\max \left\{\left|\alpha_{1}-\alpha_{2}\right|, D \varepsilon\right\}},
$$

where $\mathbf{1}_{\left|\alpha_{1}-\alpha_{2}\right|>D \varepsilon}$ is the characteristic function of the set where $\left|\alpha_{1}-\alpha_{2}\right|>D \varepsilon$. Inserting now (7.25) in (7.26) we obtain

$$
\begin{aligned}
\partial_{\bar{z}} f= & v\left(\varphi_{2}, \alpha_{2}\right) \frac{\partial_{z}\left(\varphi_{1}-\varphi_{2}\right)}{\max \left\{\left|\alpha_{1}-\alpha_{2}\right|, D \varepsilon\right\}}+\frac{\left[v\left(\varphi_{1}, \alpha_{1}\right)-v\left(\varphi_{2}, \alpha_{2}\right)\right]}{\max \left\{\left|\alpha_{1}-\alpha_{2}\right|, D \varepsilon\right\}} \partial_{z} a_{1} \\
& +\frac{\mu\left(\varphi_{1}, \alpha_{1}\right)-\mu\left(\varphi_{2}, \alpha_{2}\right)}{\max \left\{\left|\alpha_{1}-\alpha_{2}\right|, D \varepsilon\right\}}-f \mathbf{1}_{\left|\alpha_{1}-\alpha_{2}\right|>D \varepsilon} \frac{\partial_{\bar{z}}\left|\alpha_{1}-\alpha_{2}\right|}{\max \left\{\left|\alpha_{1}-\alpha_{2}\right|, D \varepsilon\right\}}
\end{aligned}
$$


From (7.27) we deduce

$$
\begin{aligned}
\partial_{\bar{z}} f= & v\left(\varphi_{2}, \alpha_{2}\right) \partial_{z} f+v\left(\varphi_{2}, \alpha_{2}\right) f \mathbf{1}_{\left|\alpha_{1}-\alpha_{2}\right|>D \varepsilon} \frac{\partial_{z}\left|\alpha_{1}-\alpha_{2}\right|}{\max \left\{\left|\alpha_{1}-\alpha_{2}\right|, D \varepsilon\right\}} \\
& +\frac{\left[v\left(\varphi_{1}, \alpha_{1}\right)-v\left(\varphi_{2}, \alpha_{2}\right)\right]}{\max \left\{\left|\alpha_{1}-\alpha_{2}\right|, D \varepsilon\right\}} \partial_{z} a_{1}+\frac{\mu\left(\varphi_{1}, \alpha_{1}\right)-\mu\left(\varphi_{2}, \alpha_{2}\right)}{\max \left\{\left|\alpha_{1}-\alpha_{2}\right|, D \varepsilon\right\}} \\
& -f \mathbf{1}_{\left|\alpha_{1}-\alpha_{2}\right|>D \varepsilon} \frac{\partial_{\bar{z}}\left|\alpha_{1}-\alpha_{2}\right|}{\max \left\{\left|\alpha_{1}-\alpha_{2}\right|, D \varepsilon\right\}} .
\end{aligned}
$$

Using now the second equation in (7.25) we obtain the existence of a constant $K_{0}>0$ such that

$$
\left|\nabla\left(\alpha_{1}-\alpha_{2}\right)\right| \leq K_{0}\left[\left|\varphi_{1}-\varphi_{2}\right|+\left|\alpha_{1}-\alpha_{2}\right|\right]
$$

This later fact gives

$$
\left|\frac{\nabla\left(\alpha_{1}-\alpha_{2}\right)}{\max \left\{\left|\alpha_{1}-\alpha_{2}\right|, D \varepsilon\right\}}\right| \leq K_{0}[|f|+1] .
$$

Combining (7.28) and (7.30) we obtain the following bound : there exists $K_{1}>0$ and $K_{2}>0$ such that

$$
\left|\partial_{\bar{z}} f-v\left(\varphi_{2}, \alpha_{2}\right) \partial_{z} f\right| \leq K_{1}[|f|+1]\left|\partial_{z} \varphi_{1}\right|+K_{2}\left[|f|^{2}+1\right] .
$$

From (7.22) we have that

$$
\begin{aligned}
& \int_{\Sigma_{\varepsilon, r} \times \mathbb{R}}\left(S_{\delta} \circ w\right)^{*}\left(d x^{1} \wedge d x^{2} \wedge d x^{3}\right) \\
& =\left(\int_{\pi\left(\Sigma_{\varepsilon, r}\right)} D^{-2}\left[\left|\partial_{z} f\right|^{2}-\left|\partial_{\bar{z}} f\right|^{2}\right] \frac{i}{2} d z \wedge d \bar{z}\right)\left(\int_{-\infty}^{+\infty} \operatorname{det}\left(D S_{\delta}\right) \circ w \lambda^{-5} d t\right) .
\end{aligned}
$$

Since $\operatorname{det}\left(D S_{\delta}\right)(y) \geq 0$ on $\mathbb{R}^{3}$,

$$
\eta(z):=\int_{-\infty}^{+\infty} \operatorname{det}\left(D S_{\delta}\right) \circ w \lambda^{-5} d t \geq 0 .
$$

Moreover we also have the following bound given by (7.23)

$$
\eta \leq C_{D}=C_{\delta}
$$


Using (7.31) we then deduce the following lower bound:

$$
\begin{aligned}
& \int_{\Sigma_{\varepsilon, r} \times \mathbb{R}}\left(S_{\delta} \circ w\right)^{*}\left(d x^{1} \wedge d x^{2} \wedge d x^{3}\right) \\
& \geq \int_{\pi\left(\Sigma_{\varepsilon, r}\right)} D^{-2}\left[1-v^{2}\left(\varphi_{2}, \alpha_{2}\right)\left|\partial_{z} f\right|^{2}\right] \eta \frac{i}{2} d z \wedge d \bar{z} \\
& \quad-\tilde{C}_{\delta} \int_{\pi\left(\Sigma_{\varepsilon, r}\right)}\left[4\left(K_{1}\right)^{2}(|f|+1)^{2}\left|\partial_{z} \varphi_{1}\right|^{2}+4\left(K_{2}\right)^{2}\left(|f|^{2}+1\right)^{2}\right] \frac{i}{2} d z \wedge d \bar{z}
\end{aligned}
$$

Using the fact that $|f(z)|=|g(\xi)| \leq D^{-1}$ on $\Sigma_{\varepsilon, r}$, and that, for $r$ small enough $\left|v\left(\varphi_{2}, \alpha_{2}\right)\right|<1 / 2$, we obtain the existence of a constant $K_{\delta}$ such that

$$
\begin{aligned}
& \int_{\Sigma_{\varepsilon, r} \times \mathbb{R}}\left(S_{\delta} \circ w\right)^{*}\left(d x^{1} \wedge d x^{2} \wedge d x^{3}\right) \\
& \geq-K_{\delta} \int_{D^{2}} \sum_{j=1}^{Q}\left[\left|\nabla \varphi_{j}\right|^{2}+1\right] \frac{i}{2} d z \wedge d \bar{z} \geq-K,
\end{aligned}
$$

with $K>0$ independent of $r$ and $\varepsilon$.

Recall now that $w=v$ on $\partial \Sigma_{\varepsilon, r} \times \mathbb{R}$. Then by Stokes theorem

$$
\begin{aligned}
\int_{\Sigma_{\varepsilon, r} \times \mathbb{R}}\left(S_{\delta} \circ w\right)^{*}\left(d x^{1} \wedge d x^{2} \wedge d x^{3}\right) & =\int_{\partial \Sigma_{\varepsilon, r} \times \mathbb{R}}\left(S_{\delta} \circ w\right)^{*}\left(\sum_{i=1}^{3} x^{j} d x^{j+1} \wedge d x^{j-1}\right) \\
& =\int_{\partial \Sigma_{\varepsilon, r} \times \mathbb{R}}\left(S_{\delta} \circ v\right)^{*}\left(\sum_{i=1}^{3} x^{j} d x^{j+1} \wedge d x^{j-1}\right) .
\end{aligned}
$$

This is the desired lower bound (7.17) and Lemma 7.8 is proved.

Proof of Lemma 7.7. The result follows straight from Lemma 7.6. Observe that, by Lemma 7.5 and by homotopy, the degree computed there is the same as the degree of the function

$$
\frac{\varphi_{i}-\varphi_{j}}{D \varepsilon}=\frac{\varphi_{i}-\varphi_{j}}{\max \left\{\left|\alpha_{i}-\alpha_{j}\right|, D \varepsilon\right\}}=g
$$

on the loop $\left\{\left|\phi_{i}-\phi_{j}\right|=\varepsilon\right\}$ around $z_{l}$. By the same computation performed in (7.32) (we can take without loss of generality $\omega=\sum_{i=1}^{3} x^{j} d x^{j+1} \wedge d x^{j-1}$ ), since the degree of $g$ is exactly $\int_{\pi\left(\Sigma_{\varepsilon, r}\right)} D^{-2}\left[\left|\partial_{z} f\right|^{2}-\left|\partial_{\bar{z}} f\right|^{2}\right] \frac{i}{2} d z \wedge d \bar{z}$, we get that the degree of $S_{\delta} \circ w$ is strictly positive. 
Proof of Theorem 7.1. We argue by contradiction. If we had countably many singularities of the form (7.1) accumulating onto 0 , around each such singular point, on $\pi^{-1}\left(\partial B_{\rho}^{2}\left(z_{l}\right)\right) \times \mathbb{R}$, we would have a strictly positive degree for $u$, thanks to Lemma 7.7. Let us observe, however, the degree of $u$ on $\partial B_{r} \times \mathbb{R}$; this is the same as the degree of $u$ on $\partial \Sigma_{r, \varepsilon} \times \mathbb{R}$, since these two 2-surfaces are homotopic and we do not cross any singularity during this homotopy (see (ii) on page 64 and recall that $u$ is smooth out of the singularities). Choosing $r$ smaller and smaller, we must then have, under the contradiction assumption, that the degree of $u$ on $\partial B_{r} \times \mathbb{R}$ goes to $-\infty$ as $r \rightarrow 0$, which contradicts Lemma 7.8 .

\section{References}

[1] JR. Almgren and J. Frederick, "Almgren's big Regularity Paper", World Scientific Monograph Series in Mathematics, 1, " $Q$-valued Functions Minimizing Dirichlet's Integral and the Regularity of Area-minimizing Rectifiable Currents up to Codimension 2", with a preface by Jean E. Taylor and Vladimir Scheffer, World Scientific Publishing Co. Inc., River Edge, NJ, 2000, xvi+955.

[2] N. ARonsZAJn, A unique continuation theorem for solutions of elliptic partial differential equations or inequalities of second order, J. Math. Pures Appl. (9) 36 (1957), 235-249.

[3] S. X.-D. CHANG, Two-dimensional area minimizing integral currents are classical minimal surfaces, J. Amer. Math. Soc. 1 (1988), 699-778.

[4] C. De Lellis and E. Spadaro, " $Q$-Valued Functions Revisited", Mem. Amer. Math. Soc., 211 (2011), n. 991, vi+79.

[5] S. K. Donaldson and R. P. Thomas, Gauge Theory in higher dimensions, In: "The Geometric Universe" (Oxford, 1996), Oxford Univ. Press, 1998, 31-47.

[6] L. C. Evans and R. F. GARIEPY, "Measure Theory and Fine Properties of Functions", Studies in Advanced Mathematics, CRC Press, Boca Raton, FL, 1992, viii+268.

[7] H. Federer, "Geometric Measure Theory", Die Grundlehren der mathematischen Wissenschaften, Band 153, Springer-Verlag New York Inc., New York, 1969, xiv+676.

[8] M. Giaquinta, G. ModicA and J. SOUČEK, "Cartesian Currents in the Calculus of Variations. I", Ergebnisse der Mathematik und ihrer Grenzgebiete. 3. Folge. A Series of Modern Surveys in Mathematics [Results in Mathematics and Related Areas. 3rd Series. A Series of Modern Surveys in Mathematics], 37, Cartesian currents, Springer-Verlag, Berlin, 1998, xxiv+711.

[9] D. Gilbarg and N. S. Trudinger, "Elliptic Partial Differential Equations of Second Order", Classics in Mathematics, Reprint of the 1998 edition, Springer-Verlag, Berlin, 2001, xiv+517.

[10] R. Harvey and H. B. JR. Lawson, Calibrated geometries, Acta Math. 148 (1982), 47157.

[11] M. Haskins, Special Lagrangian cones, Amer. J. Math. 126 (2004), 845-871.

[12] D. D. JOYCE, "Riemannian Holonomy Groups and Calibrated Geometry", Oxford Graduate Texts in Mathematics, 12, Oxford University Press, Oxford, 2007, x+303.

[13] M. J. MiCALLEF and B. WhITE, The structure of branch points in minimal surfaces and in pseudoholomorphic curves, Ann. of Math. (2) 141 (1995), 35-85.

[14] F. Morgan, "Geometric Measure Theory", Fourth edition, A beginner's guide, Elsevier/Academic Press, Amsterdam, 2009, viii+249.

[15] C. B. JR. MORREY, "Multiple Integrals in the Calculus of Variations", Die Grundlehren der mathematischen Wissenschaften, Band 130, Springer-Verlag New York, Inc., New York, 1966, ix+506. 
[16] D. Pumberger and T. Rivière, Uniqueness of tangent cones for semi-calibrated 2cycles, Duke Math. J. 152 (2010), 441-480.

[17] T. RIVIÈRE and G. TIAN, The singular set of J-holomorphic maps into projective algebraic varieties, J. Reine Angew. Math. 570 (2004), 47-87. 58J45.

[18] T. RIVIÈRE and G. TIAN, The singular set of 1-1 integral currents, Ann. of Math. (2) 169 (2009), 741-794.

[19] L. Simon, "Lectures on Geometric Measure Theory", Proceedings of the Centre for Mathematical Analysis, Australian National University, 3, Australian National University Centre for Mathematical Analysis, Canberra, 1983, vii+272.

[20] J. Simons, Minimal varieties in riemannian manifolds, Ann. of Math. (2) 88 (1968), 62105.

[21] C. H. Taubes, "SW $\Rightarrow$ Gr: From the Seiberg-Witten Equations to Pseudo-holomorphic Curves". Seiberg Witten and Gromov invariants for symplectic 4-manifolds., 1-102, First Int. Press Lect. Ser., 2, Int. Press, Somerville, MA, 2000.

[22] G. TIAN, Gauge theory and calibrated geometry. I, Ann. of Math. (2) 151 (2000), 193-268.

[23] B. WhITE, Tangent cones to two-dimensional area-minimizing integral currents are unique, Duke Math. J. 50 (1983), 143-160.

ETH, Zürich

Departement Mathematik

Rämistrasse, 101

8092 Zürich, Switzerland

cbellett@math.princeton.edu 